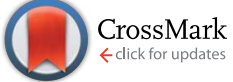

Cite this: J. Mater. Chem. A, 2015, 3, 22507

Received 23rd July 2015 Accepted 25th September 2015

DOI: $10.1039 / \mathrm{c} 5 \mathrm{ta} 05666 \mathrm{~g}$

www.rsc.org/MaterialsA

\title{
Recent progress in micro-scale energy storage devices and future aspects
}

\begin{abstract}
Ankit Tyagi $\uparrow^{a}$ Kumud Malika Tripathi $\dagger^{a}$ and Raju Kumar Gupta*b
Recent developments in the field of energy storage materials are expected to provide sustainable solutions to the problems related to energy density and storage. The increasing energy demand for next generation portable and miniaturized electronic devices has sparked intensive interest to explore micro-scale and lightweight energy storage devices. This critical review provides an overview of the state-of-the-art recent research advances in micro-scale energy storage devices for supercapacitors (SCs), as well as their future importance in technology. Much effort has been devoted to fabricate high performance, ultrathin, planar, solid state and flexible micro-supercapacitors (MSCs) with aesthetic appeal. Nanomaterials for MSCs, their fabrication techniques and performances are critically analyzed. The technical challenges and perspectives for MSCs are also discussed. The review concludes with few suggestions for future advancements in this area.
\end{abstract}

\section{Introduction}

With the continuous escalating global population and economy, energy crisis has turned into one of the most crucial problems due to overusage of conventional resources. In this regard, microfluidic energy technologies are emerging as the

${ }^{a}$ Department of Chemical Engineering, Indian Institute of Technology Kanpur, Kanpur 208016, India

${ }^{b}$ Department of Chemical Engineering and Thematic Unit of Excellence on Soft Nanofabrication, Indian Institute of Technology, Kanpur 208016, U.P., India. E-mail: guptark@iitk.ac.in; Fax:+91-5122590104; Tel: +91-5122596972

$\uparrow$ Contributed equally. future technology for energy processes. ${ }^{1}$ There is an urgent need for renewable energy storage systems due to the disruptive potential of conventional energy resources. Tremendous breakthroughs in nanotechnology lead to nano-revolutions in energy storage devices.

Supercapacitors (SCs), also known as ultracapacitors, are one of the currently available potential energy storage devices with high power density and fast charge discharge rates that can stand alone or in integration with other devices such as batteries in order to fulfill the energy requirement. ${ }^{2-4}$ They can deliver high power in a very short period of time and exhibit long cycle life. ${ }^{5}$ These have emerged as a substitute for batteries and conventional capacitors. In contrast to conventional

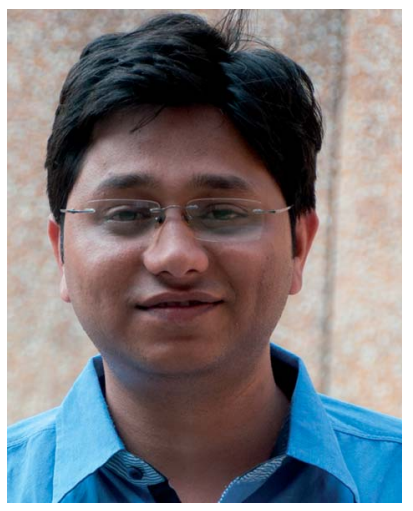

Ankit Tyagi received his Bachelors degree in Chemical Engineering from the Institute of Engineering and Technology Lucknow in 2010 and Masters degree in Chemical Engineering from the Indian Institute of Technology Roorkee in 2012. He has been pursuing his doctoral degree at the Department of Chemical Engineering, Indian Institute of Technology Kanpur, India since 2012. His current research interests focus on the development of metal oxide nanostructures for high energy density supercapacitor applications.

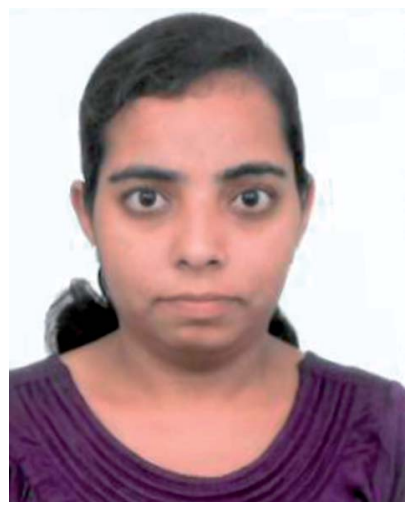

Dr Kumud Malika Tripathi received her Ph.D. from the Department of Chemistry at the Indian Institute of Technology, Kanpur, India, in 2013. After completing her Ph.D. she was a postdoctoral scientist in the group of Prof. Jean-Francois Feller (Smart Plastic Group) at the University of South Brittany, Lorient, France. Currently, she is working in the group of Dr Raju Kumar Gupta, Department of Chemical Engineering, IIT Kanpur, India. Her research focus is on the fabrication of carbon nanostructures with diverse morphologies through greener routes and their nanocomposites for multifunctional applications from biomedical to energy harvesting. 
capacitors, SCs provide several orders higher magnitude of energy density and uptake pulse. ${ }^{4}$ The first patent on SCs came into existence in 1957, when Becker from General Electric Corp. proposed a carbon (high surface area) coated metallic current collector in $\mathrm{H}_{2} \mathrm{SO}_{4}$ solution. It was believed that energy was being stored in the pores of carbon. ${ }^{6}$ Sohio was the first to commercialize SCs in $1969 .^{7-9}$ During the period of 1975 to 1981 , a new concept of energy storage in SCs was proposed by Conway. ${ }^{10} \mathrm{SCs}$ were fabricated by using $\mathrm{RuO}_{2}$ film in aqueous $\mathrm{H}_{2} \mathrm{SO}_{4}$ with high specific capacitance and low internal resistance.$^{\mathbf{1 0}} \mathrm{He}$ proposed that energy was stored in a non-faradaic process due to redox reactions. SCs became prominent in 1990s, when their potential applications were found in hybrid electric vehicles. ${ }^{7,8}$ Depending upon the charge storage mechanism at the electrode/electrolyte interface SCs can be classified into three categories, namely electrical double layer capacitors (EDLCs), pseudocapacitors and hybrid SCs. In the case of EDLCs electrodes are generally made of high surface area carbon materials having a mesoporous structure. ${ }^{2}$ At the electrode/electrolyte interface electrostatically reversible adsorption of electrolyte ions takes place due to which a double layer of charges is formed. The thickness of such a double layer is in the order of angstroms $(\AA)$ with a very high interfacial area and leads to the high specific capacitance of SCs. ${ }^{\mathbf{8 1 1}, 12}$ Since there is no chemical reaction involved between the electrode and electrolyte, the process is highly reversible and millions of charge/discharge cycles are possible, thus resulting in the long life time of the EDLCs. ${ }^{13,14}$ Current in the external circuit flows due to the formation and deformation of the double layer. ${ }^{13,14}$ Electrical energy is stored in pseudocapacitors due to the faradaic charge transfer between the electrode and electrolyte. Porosity of the electrode material is a key factor for charge storage in EDLCs. ${ }^{15}$ All the pores present in the electrode material are not accessible for accommodating electrolyte ions and hence are not electrochemically active. ${ }^{\mathbf{1 6}}$ Pores larger than $5 \AA$ are easily accessible to electrolyte ions and the optimum size of the pores is found to be

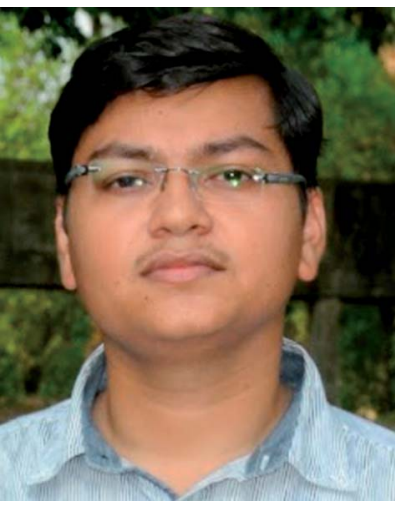

Dr Raju Kumar Gupta is an Assistant Professor at the Department of Chemical Engineering, Indian Institute of Technology Kanpur, India. He completed his Ph.D. at the Department of Chemical and Biomolecular Engineering, National University of Singapore, Singapore in 2010. His research focuses on the synthesis of quantum dots utilizing greener routes for photocatalysis and solar energy applications, synthesis of water soluble photoluminescent carbon nanodots from biowaste and developing their metal oxide hybrid nanostructures for supercapacitor applications. He is the recipient of several fellowships and awards such as IEI Young Engineer Award (2014-15) and DST Inspire Faculty Award 2013.
7-8 A for both aqueous and organic electrolytes. ${ }^{17}$ Hence, the size of the electrolyte ion should be compatible with the pore size in order to obtain maximum specific capacitance. Mesoporous carbons (pore size $2-50 \mathrm{~nm}$ ) are considered as the best electrode material for the fabrication of EDLCs., ${ }^{3,18}$ Surface functionalities are another important parameter for the determination of specific capacitance in EDLCs. ${ }^{19}$ The surface oxygenous groups are found to enhance the specific capacitance in the EDLCs. ${ }^{15}$

Energy is stored in pseudocapacitors because of faradaic charge transfer between the electrode and electrolyte. ${ }^{\mathbf{1 1}}$ Pseudocapacitors show 10-100 times higher specific capacitance than EDLCs. However, pseudocapacitors usually exhibit low power densities and cycling life than EDLCs. ${ }^{\mathbf{1 1 , 1 3 , 1 4 , 2 0 - 2 2}}$ Here, charge transfer occurs due to oxidation-reduction, electrosorption, intercalation and the doping-dedoping process. Pseudocapacitive materials such as transition metal oxides and conducting polymers are used as active electrode materials.,23 The working voltage for the pseudocapacitors is higher than that of EDLCs. ${ }^{23}$ It has been observed that electrochemical faradaic processes occur close to the surface only and a thin layer is responsible for the charge stored in pseudocapacitors. ${ }^{24}$

In the past few years researchers have developed SCs with an asymmetrical configuration: a carbon material having double layer capacitance as one electrode and a pseudocapacitive material, e.g. a metal oxide, with faradaic charge storage as the other electrode. SCs with such a configuration are known as hybrid SCs. Usage of two types of electrodes is advantageous in terms of enhanced energy density, lightweight and simultaneous retention of flexibility. In hybrid SCs since a pseudocapacitive electrode is used to store the charge, it helps in improving the overall specific capacitance and also extends the working voltage of the hybrid electrochemical capacitors. ${ }^{11,13,14,22}$ Hybrid electric vehicles, back-up power systems, uninterruptible power supplies for computers and industrial energy management systems are some of the potential applications of SCs. However, the limited surface area of the electrode materials offers lower volumetric capacitance in SCs, restricting their further use in modern technological devices.

The recent technological trends towards miniaturization of energy storage devices are accelerating the requirement for high-performance portable microelectronics and small-scale energy storage units. Miniaturization of SCs at the microscopic level has attracted tremendous interest in recent years as a potential solution to this demand for energy harvesting. ${ }^{25,26}$ Miniaturization of energy storage devices significantly increases the device density and can deliver energy at higher efficiency. ${ }^{27}$ The advent of modern microelectronic devices such as microsupercapacitors (MSCs) fulfills the requirement of high power delivery in a very short period of time. MSCs, a novel class of recently introduced miniaturized micro/nanoscale electrochemical energy storage devices, are attracting immense interest owing to their unique features. In contrast to conventionally used batteries and SCs, MSCs offer several orders higher power density, long cycle life and large rate capability, and are environment-friendly as well. ${ }^{28}$ More significantly, onchip microscale devices such as micro-sensors, micro-batteries, 
biomedical implants and energy harvesting microsystems can be directly integrated or coupled with MSCs and would have the advantage of excellent peak power..$^{25,29,30}$ Recent advances have been made for patterning of the complex architecture of interdigitated 2D (two-dimensional) microscale energy storage devices or semi-3D devices. However, fabrication of 3D microscale devices is still at the infant stage and requires immediate attention. ${ }^{25,26,31,32}$ Several micro-fabrication techniques are in practice for the efficient miniaturization and interdigitation of SCs. ${ }^{33,34}$ Interdigitated planar MSCs have the advantage of quick ionic diffusion in contrast to traditionally used membrane based SCs. ${ }^{35}$ However, fabrication of suitable electrode materials and their application in MSCs as an active material while maintaining excellent performance at the micro/nanoscale are key issues and still need to be addressed. Apart from this, the complexity of the patterning technique and non-scalability are also the main shortcomings of the current technologies. ${ }^{36}$

Various micro-fabrication technologies such as sputtering, chemical vapor deposition (CVD), layer-by-layer (LBL) deposition, ink-jet printing, ${ }^{37}$ electrochemical/electrophoretic deposition, spray deposition and lithography are in use for the miniaturization and integration of MSC devices. ${ }^{38}$ Planar SCs have potential application in stretchable/flexible electronic devices such as touchscreens, smart sensors and actuators. ${ }^{30}$ One of the main concerns in the use of MSCs is that energy and power densities need to be increased either by improving material properties or by designing the appropriate architecture of the devices. ${ }^{38-40}$ Another promising approach is to directly fabricate interdigitated on-chip fingers by using the lift-off photoresist technique on a flexible surface such as a polyethylene terephthalate (PET) substrate. It has been reported that introduction of flexibility in solid-state MSCs would open a new window for their applications in strain-related environments. Interdigitated structures for on-chip design or integration of small-scale devices are required for the fabrication of MSCs compatible with microelectronic technologies.

The increasing demand for multifunctional portable, wearable and rolled up electronic devices has attracted immense interest for the fabrication of flexible energy storage devices. ${ }^{\mathbf{4 1}}$ MSCs are considered as the most promising power source for microscale and wearable electronic devices due to their high power density, high efficiency and long cycle life. Ecological concerns and a breakthrough in fabrication of low cost carbon nanomaterials may bring a revolution in fabrication techniques of MSCs. Herein we focus on the latest advancements in microscale energy storage devices, their applications in the practical world and future prospectives. We have critically selected the forefront of advanced energy research, technological progress and potential application in the area of miniaturized energy storage devices. Techniques for the fabrication of interdigitated electrodes are discussed in brief. Active electrode materials along with their performance are summarized. The new trends in materials development and characterization, physico-chemical and electrochemical interaction of nanomaterials and future challenges are also mentioned.

\section{Current advances in energy storage}

One of the major challenges in the present times is the identification of high-efficiency energy storage devices that do not emit any harmful exhaust. SCs and batteries are widely used electrochemical devices for energy storage and play a crucial role in our daily life. Among numerous energy storage devices currently in use, MSCs have subjugated the portable and flexible electronic market as a next generation energy storage device. Recent trends in the design and fabrication of such MSCs were reviewed last year by Gogotsi and coworkers. ${ }^{38}$ Research and development of MSCs consuming less power have turned into recent research advancements. Fabrication of high performance MSCs with flexibility and stability greatly depends upon the electrochemical and mechanical properties of the electrode material. Hence it is still a challenge to find out low-cost, appropriate nanomaterials for application as electrode materials in MSCs.

Solid state SCs have emerged as a complement to classical SCs due to their superior performance in terms of power density and ability to work under harsh environmental conditions, especially in space, automotive and bio-implantable systems. ${ }^{42,43}$ Akin to conventional SCs, solid state SCs consist of flexible electrodes, packing materials, separators and electrolytes in the solid state. ${ }^{30}$ In contrast to classical SCs, only surface charges are used to accumulate power in solid state SCs which store charges in electrical double layers. Solid state electrolytes can significantly achieve high efficiency energy delivery and enhance the stability and rate capability due to their wider operational voltage window. ${ }^{\mathbf{4 4}}$ The higher ionic conductivity and low viscosity further enhance the ion transport between the electrode surface and electrolyte. ${ }^{44}$ Moreover, the major problem of electrolyte leakage is solved significantly and consequently reduces the packing cost. Gel polymer electrolytes such as PVA, and $\mathrm{H}_{3} \mathrm{PO}_{4}$ or $\mathrm{H}_{2} \mathrm{SO}_{4}$ gels are extensively used as solid electrolytes supported by flexible substrates. ${ }^{45}$ Planar MSCs, exhibiting planar electrode geometry within a confined surface area, belong to both solid state and flexible MSCs depending upon the substrate material. ${ }^{\mathbf{4 6}}$ Recent research advancement reveals that planar MSCs can be integrated with autonomous micro-sensor networks due to their high performance and extreme cyclic stability. ${ }^{3}$

Despite the immense progress in flexible and 3D MSCs, there is still broad scope for research and development in this field. For instance MSCs having solid electrolytes can restrict passage of sweat from human skin and thus can find their prospective applications in wearable microelectronic devices. ${ }^{47}$ In this regard, fiber based supercapacitors hold great potential. ${ }^{47}$ The unique architectures of 1D fiber-based SCs allow their rendering in a variety of substrate materials with versatile localization at different places. The basic performance, evaluation and current state-of-the-art progress in fiber based MSCs are summarized by Chen and co workers. ${ }^{47}$

Miniaturization of electronic devices created a great demand for smaller size and high energy density microscale energy 
storage devices. ${ }^{48}$ Among the energy storage devices Li-ion batteries exhibit the highest energy density and still show great promise for the development of high performance Li-ion microbatteries. Flexible, small unit and shape-conformable lithium-ion batteries have aroused immense interest in the past few years. Microbatteries have potential applications in various electronic devices such as micromachines, microsensors, smart cards, transmitters, actuators, drug delivery systems, pacemakers, hearing aids, defibrillators, and in vivo imaging. ${ }^{48-50}$ The architecture as well as the choice of electrode material in microbatteries is different from that of conventional batteries. Owing to the smaller size, a planar design is not useful since it limits the energy density and areal power. In this regard, a 3D out-of-plane design is found to be appropriate for increasing both the energy density and areal power in microbatteries. One of the challenging issues for introducing flexibility in lithiumion batteries is the synthesis/selection of bendable current collectors exhibiting good mechanical properties and fabrication of a suitable electrode material. Last year Shen and coworkers reviewed the current trends towards the fabrication of flexible energy storage devices for both lithium-ion batteries and supercapacitors. ${ }^{51}$ They further addressed the technological advancements made in CNPs and their composite-based electrode materials in flexible MSCs. The integration of lithium-ion batteries and MSCs with other units in order to achieve selfpowered systems for advanced applications was also reviewed.

Ferrari and her co-workers reviewed the manufacturing strategies of $3 \mathrm{D} \mathrm{Li}$-ion microbatteries. ${ }^{48}$ The operating mechanism for microbatteries and conventional Li-ion batteries is almost similar. Since a separator is not required for all solid state microbatteries, the overall mass of the cell decreases with increase in per unit mass energy density. With decrease in size the diffusion path of the ions also decreases and the ionic and electronic conduction predominantly depend on the electrode material. The thickness of the active layers (electrode and electrolyte) also contributes to the internal cell resistance, since with increase in thickness the overall resistance of the cell increases. Strategies for the selection of materials and electrochemical couples have been discussed by Wang et al. in their recent review. ${ }^{49} 3 \mathrm{D}$ nickel sulphide with a hollow structure as an electrode material was synthesized by Lee et al. in order to enhance the areal capacity. The areal capacity of such fabricated microbatteries was found to be $1.5 \mathrm{~mA} \mathrm{~h} \mathrm{~cm} \mathrm{~cm}^{-2}$ at a current density of $0.5 \mathrm{~mA} \mathrm{~cm}{ }^{-2} .{ }^{52}$ Westover et al. fabricated an on-chip Li-ion microbattery with a porous Si material passivated with graphene. ${ }^{53}$ The device exhibited excellent cycling performance for more than 10000 cycles without much capacity fading. Inkjet printed porous $\mathrm{LiFePO}_{4}$ was used as the positive electrode material for the Li-ion microbatteries. The devices showed a specific capacity of $80 \mathrm{~mA} \mathrm{~h} \mathrm{~g}^{-1}$ at a cycling rate of $9 \mathrm{C}$ with no significant loss in specific capacity even after 100 cycles. ${ }^{50}$ The ink-jet printing technique was also used by Delannoy et al. for the synthesis of $\mathrm{LiFePO}_{4}$ and $\mathrm{Li}_{4} \mathrm{Ti}_{5} \mathrm{O}_{12}$ porous composite electrodes for Li-ion microbatteries. The thus-fabricated device exhibited a surface capacity of $300 \mu \mathrm{A} \mathrm{h} \mathrm{cm}{ }^{-2}$ for 100 cycles. $^{54}$ Liu et al. adapted the electrodeposition technique for the fabrication of a 3D metallic Si electrode over a Ni inverse opal structure. They observed a lower internal resistance for the $\mathrm{Li}$ ion diffusion for the Si-on-Ni opal structure than the Si-on-Ni nanocables. ${ }^{55}$ A 3D Si scaffold was synthesized by Lethien and co-workers for microbatteries. ${ }^{56} \mathrm{~A} \mathrm{TiO}_{2}$ layer was deposited over a Si scaffold by the atomic layer deposition technique. The authors obtained a surface capacity of $74 \mu \mathrm{A} \mathrm{h} \mathrm{cm} \mathrm{cm}^{-2}$ at a discharge rate of $\mathrm{C} / 2$ for the $38 \mathrm{~nm}$ thick $\mathrm{TiO}_{2}$ layer. The surface capacity reached $0.2 \mathrm{~mA} \mathrm{~h} \mathrm{~cm} \mathrm{~m}^{-2}$ at a discharge rate of $\mathrm{C} /$ 10, for a $\mathrm{TiO}_{2}$ layer deposition thickness of $150 \mathrm{~nm}$.

Herein, we basically focus on the recent advancements in the materials and fabrication techniques of interdigitated electrodes for MSCs in contrast to microscale lithium-ion batteries. Techniques for the fabrication of interdigitated electrodes are discussed in brief, followed by a summary of active electrode materials and their performances.

\section{Fabrication technique of electrodes for supercapacitors and micro-supercapacitors}

The performances of MSCs are greatly affected by the intrinsic property of the material as well as the design of the electrode pattern. Active surface area and pore size matching with the size of the electrolyte ions are some of the crucial parameters in order to enhance MSCs' power and energy density. Hence selection of a suitable electrolyte and design of appropriate electrode patterns are the key steps for the fabrication of next generation smart SCs. The interdigitated electrode is a special architecture of planar electrodes which consist of several microelectrodes (generally termed as "fingers") arranged interdigitally. The ease of control of the inter-electrode distance facilitates in-plane integration. Moreover in solid state or flexible MSCs, the electrode weight is negligible in comparison to other components and is not compatible with traditional fabrication processes. Technical aspects generally used for patterning of the architecture of MSCs are discussed here. Inkjet, screen printing, laser writing and conventional lithography are significant techniques used for the fabrication of MSCs.

\subsection{Printed micro-supercapacitors}

Printed MSCs have become the state of the art in micro-scale energy storage devices over the past few years since they offer reduction in size and bring significant advantage for industrial applications and commercial viability. ${ }^{57-60}$ Printed energy storage devices can easily be produced on a large scale.

3.1.1 Ink-jet printing. Inkjet printing is a direct printing technique, which provides control over the architecture, localization, thickness and electrical conductivity of printed electrodes on a variety of substrates. ${ }^{61}$ It is extensively used for the fabrication of conductive patterns and also in thin film organic transistors, solar cells, sensors, electrochemical energy storage devices, light-emitting devices, memory and magnetic devices, etc. In contrast to photo-lithography and other conventionally used printing techniques (screen printing), inkjet printing offers great promise due to its simple, versatile nature, low cost 
and non-contact deposition method. ${ }^{62}$ It is suitable for the large scale roll-to-roll mass production system. ${ }^{58}$ For instance, Wang et al. fabricated an asymmetric supercapacitor (ASC) by using an HP Deskjet 1010 inkjet printer. Multi-walled carbon nanotubes (MWCNTs) combined with Ag nanoparticle ink was used to fabricate the negative electrode whereas $\mathrm{MnO}_{2}$ nanoparticles ( 60-90 nm) mixed with MWCNTs/Ag ink were used to fabricate the positive electrode. The electrode pattern with the material and printer is shown in Fig. 1. The as-fabricated ASC (1 $\times 1 \mathrm{~cm}^{2}$ ) exhibited a high energy density of $1.28 \mathrm{~mW} \mathrm{~h} \mathrm{~cm}^{-3}$ and power density of $96 \mathrm{~mW} \mathrm{~cm} \mathrm{~cm}^{-3}$ at $1.8 \mathrm{~V}$ of operating potential window in LiCl electrolyte. It showed good cycling stability of $96.9 \%$ after 3000 cycles at a current density of $0.142 \mathrm{~A} \mathrm{~cm}^{-3}$. The patterned electrode also showed good bending stability and low sheet resistance. ${ }^{60}$

In a similar report Pang et al. fabricated flexible all solid state asymmetric micro-supercapacitors (AMSCs) on a PET substrate by using a Fujifilm Dimatix 3000 ink-jet printer and $\mathrm{KOH}-$ polyvinyl alcohol (PVA) gel as the electrolyte material. For the first time they used lamellar potassium cobalt phosphate hydrate $\left(\mathrm{K}_{2} \mathrm{Co}_{3}\left(\mathrm{P}_{2} \mathrm{O}_{7}\right)_{2} \cdot 2 \mathrm{H}_{2} \mathrm{O}\right)$ nanocrystal whiskers as the cathode material and graphene nanosheets as the anode material (Fig. 2). The as-fabricated MSCs showed a specific capacitance of $6 \mathrm{~F} \mathrm{~cm}^{-3}$ at a current density of $10 \mathrm{~mA} \mathrm{~cm} \mathrm{~cm}^{-3}$ and remained at $3.9 \mathrm{~F} \mathrm{~cm}^{-3}(68.1 \%)$ when the current density was increased up to $100 \mathrm{~mA} \mathrm{~cm} \mathrm{~cm}^{-3}$. Cycling was performed at $10 \mathrm{~mA}$ $\mathrm{cm}^{-3}$ for 5000 charge-discharge cycles, which showed $94.4 \%$ retention of volumetric capacitance. The maximum volumetric energy density of the device was found to be $0.96 \mathrm{~mW} \mathrm{~h} \mathrm{~cm}^{-3}$ at a potential window of $1.07 \mathrm{~V}^{61}$

$\mathrm{Xu}$ et al. also used the inkjet printing technique with graphene/polyaniline (PPy) inks by using $1 \mathrm{M} \mathrm{H}_{2} \mathrm{SO}_{4}$ as the electrolyte and obtained a maximum specific capacitance of $82 \mathrm{~F}$ $\mathrm{g}^{-1}, 2.4 \mathrm{~W} \mathrm{~h} \mathrm{~kg}^{-1}$ energy density and $124 \mathrm{~kW} \mathrm{~kg}^{-1}$ power density at a scan rate of $20 \mathrm{mV} \mathrm{s}^{-1}$ at $1 \mathrm{~V}$ potential window. ${ }^{58}$ Aqueous

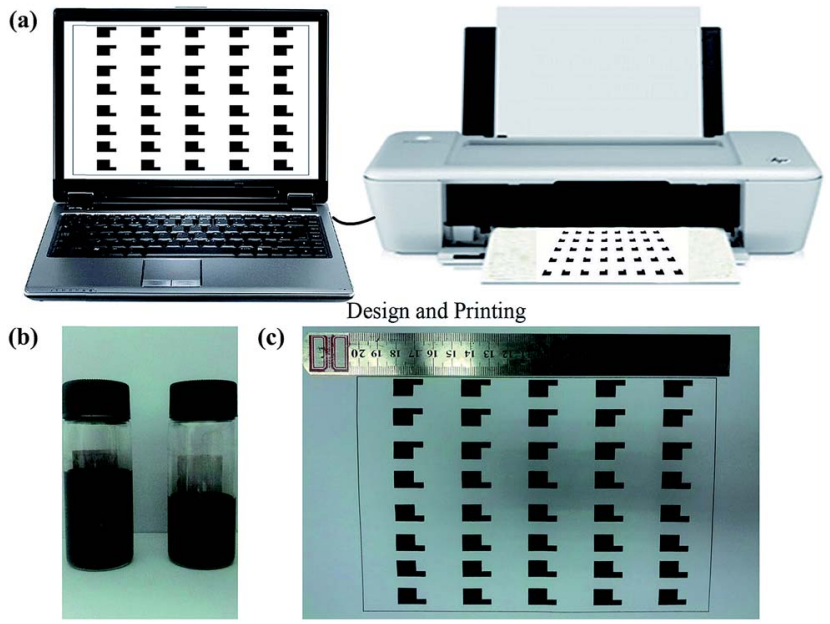

Fig. 1 Overview of the fabrication process for inkjet-printing: (a) design using a personal computer and inkjet-printing using a home printer, (b) the well-dispersed ink for inkjet-printing, (c) a photo of patterned electrodes. ${ }^{60}$ Adopted from ref. 60 with permission from The Royal Society of Chemistry.

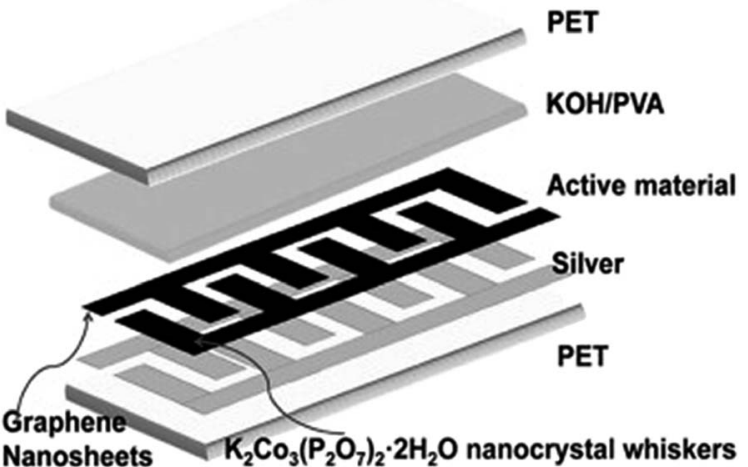

Fig. 2 Schematic view of the printed flexible micro-device. ${ }^{61}$ Adopted from ref. 61 with permission from Elsevier.

dispersion of graphene oxide (GO) was used by Matthew Ervin et al. as the ink for fabricating inkjet printed flexible SCs $(1 \times 1$ $\mathrm{cm}^{-2}$ ) over a Kapton substrate with or without fluorinated ethylene propylene coatings. GO was thermally reduced and coated onto a conductive graphene electrode. A specific capacitance of $73 \mathrm{~F} \mathrm{~g}^{-1}$ was obtained at $20 \mathrm{mV} \mathrm{s}^{-1}$ scan rate at a potential window of $0-3 \mathrm{~V}$ with $\mathrm{BMIM}^{\mathrm{BF}} \mathrm{B}_{4}$ electrolyte along with an energy density of $5.5 \mathrm{~W} \mathrm{~h} \mathrm{~kg}^{-1}$ at $0.25 \mathrm{~A} \mathrm{~g}^{-1}$ and a power density of $19 \mathrm{~kW} \mathrm{~kg}^{-1}$ at a current density of $10 \mathrm{~A} \mathrm{~g}^{-1} .^{62} \mathrm{Jung}$ et al. also fabricated a highly porous pattern of interdigitated flexible electrodes over a PET substrate for MSCs by using GO ink through inkjet printing. ${ }^{63} \mathrm{GO}$ was in-depth reduced to graphene by a photo-thermal reduction technique with a xenon flash camera of $1.50 \mathrm{~J} \mathrm{~cm}^{-2}$ intensity. A maximum volumetric

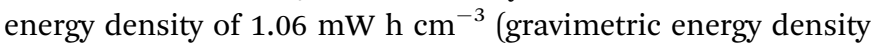

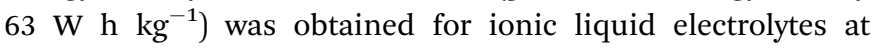
a power density of $0.408 \mathrm{~W} \mathrm{~cm}^{-3}$ (gravimetric power density $25.5 \mathrm{~kW} \mathrm{~kg}^{-1}$ ). Chi et al. used inkjet printing to prepare freestanding graphene paper for the synthesis of flexible all solid state SCs. They took commercial paper and coated it with GO using an inkjet printer and then printed this paper with graphene hydrogel-polyaniline (GH-PANI) ink (Fig. 3). After that HI solution was used to reduce GO paper to graphene paper (GP). $\mathrm{CO}_{2}$ bubbles were used to separate the freestanding film of GH-PANI/GP from the commercial paper. Graphene paper interacts with GH-PANI via $\pi-\pi$ interaction and offers mechanical strength to the freestanding film. The maximum specific capacitance of the GH-PANI/GP electrode based on mass was found to be $864 \mathrm{~F} \mathrm{~g}^{-1}$ at a current density of $1 \mathrm{~A} \mathrm{~g}^{-1}$ and the areal capacitance was found to be $190.6 \mathrm{mF} \mathrm{cm}^{-2}$ at a current density of $0.5 \mathrm{~mA} \mathrm{~cm} \mathrm{~cm}^{-2}$. Up to $96 \%$ specific capacitance was retained after 1000 cycles at a current density of $8 \mathrm{~A}$ $\mathrm{g}^{-1}$. The symmetric SC based on the GH-PANI/GP electrode and PVA- $\mathrm{H}_{2} \mathrm{SO}_{4}$ gel electrolyte showed an energy density of $24.02 \mathrm{~W}$ $\mathrm{h} \mathrm{kg} \mathrm{kg}^{-1}$ at a power density of $400.3 \mathrm{~W} \mathrm{~kg}^{-1}$ at an operating potential window of $0.8 \mathrm{~V} .^{64}$

3.1.2 Screen printing technique. In the screen printing technique a desired pattern is obtained by using a woven mesh to support an ink-blocking stencil. In this technique, nanoparticles containing inks of various materials can be directly 


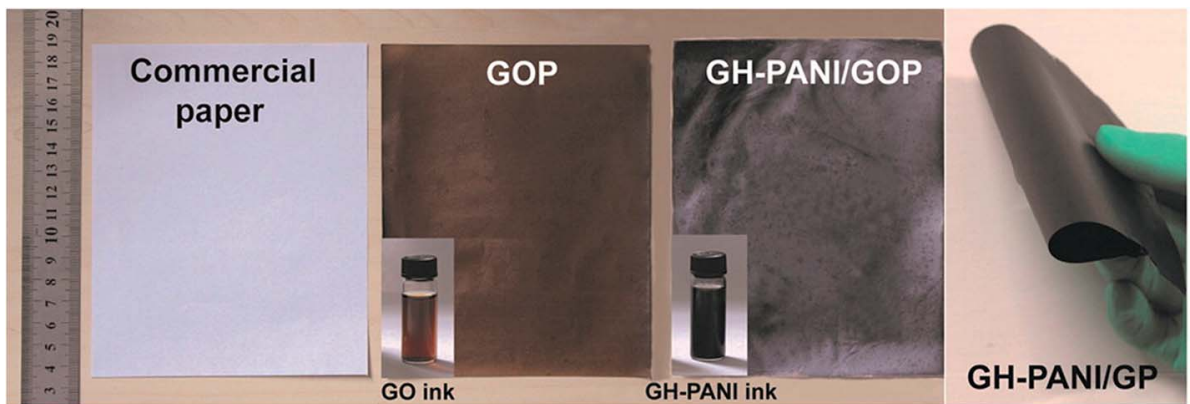

Fig. 3 Photographs of different graphene-based inks and macroscopic nanohybrid paper materials. ${ }^{64}$ Adopted from ref. 64 with permission from the American Chemical Society.

pressed against the opening of the mesh and patterns can be made over a variety of substrates, such as cloth and paper. ${ }^{59}$ Screen printing enables deposition and patterning of an electrode at the same time and thus reduces the processing time and complexity. ${ }^{65}$ Screen printing can be used to synthesize patterns within a short time at a large scale with uniform thickness. ${ }^{66}$ Recently Mensing et al. adapted the screen printing technique to prepare electrodes of surfactant stabilized graphene in combination with PANI nanofibers (PANI-Nf) over stainless steel current collectors and used them as active electrodes in SCs. ${ }^{67}$ They used $100 \mu \mathrm{m}$ thick polyester masks having a $1.5 \mathrm{~cm}$ diameter circular area and obtained $5.7 \mathrm{mg} \mathrm{cm}{ }^{-2}$ area loading of active material. The maximum specific capacitance was reported to be $690 \mathrm{~F} \mathrm{~g}^{-1}$ for the symmetric supercapacitors (SSCs) at a current density of $1 \mathrm{~A} \mathrm{~g}^{-1}$ and $0-0.8 \mathrm{~V}$ voltage window in $2 \mathrm{M} \mathrm{H}_{2} \mathrm{SO}_{4}$ aqueous electrolyte. Liu et al. screen printed a nitrogen doped reduced graphene oxide (rGO) interdigitated electrode for the fabrication of flexible all solid state MSCs by using PVA- $\mathrm{H}_{3} \mathrm{PO}_{4}$ as the gel electrolyte. ${ }^{65}$ The as-fabricated MSCs showed $3.4 \mathrm{mF} \mathrm{cm}^{-2}$ areal specific capacitance at $20 \mu \mathrm{A}$ $\mathrm{cm}^{-2}$ current density along with $0.3 \mathrm{~mW} \mathrm{~h} \mathrm{~cm}{ }^{-3}$ volumetric energy density at a power density of $0.2 \mathrm{~W} \mathrm{~cm}^{-3}$. Galvanostatic charging-discharging (GCD) was done at a current density of $100 \mu \mathrm{A} \mathrm{cm}{ }^{-2}$ which showed $98.4 \%$ retention of the initial specific capacitance. Screen printing was used by Sudhakar et al. to fabricate flexible electrodes of rGO derived from used
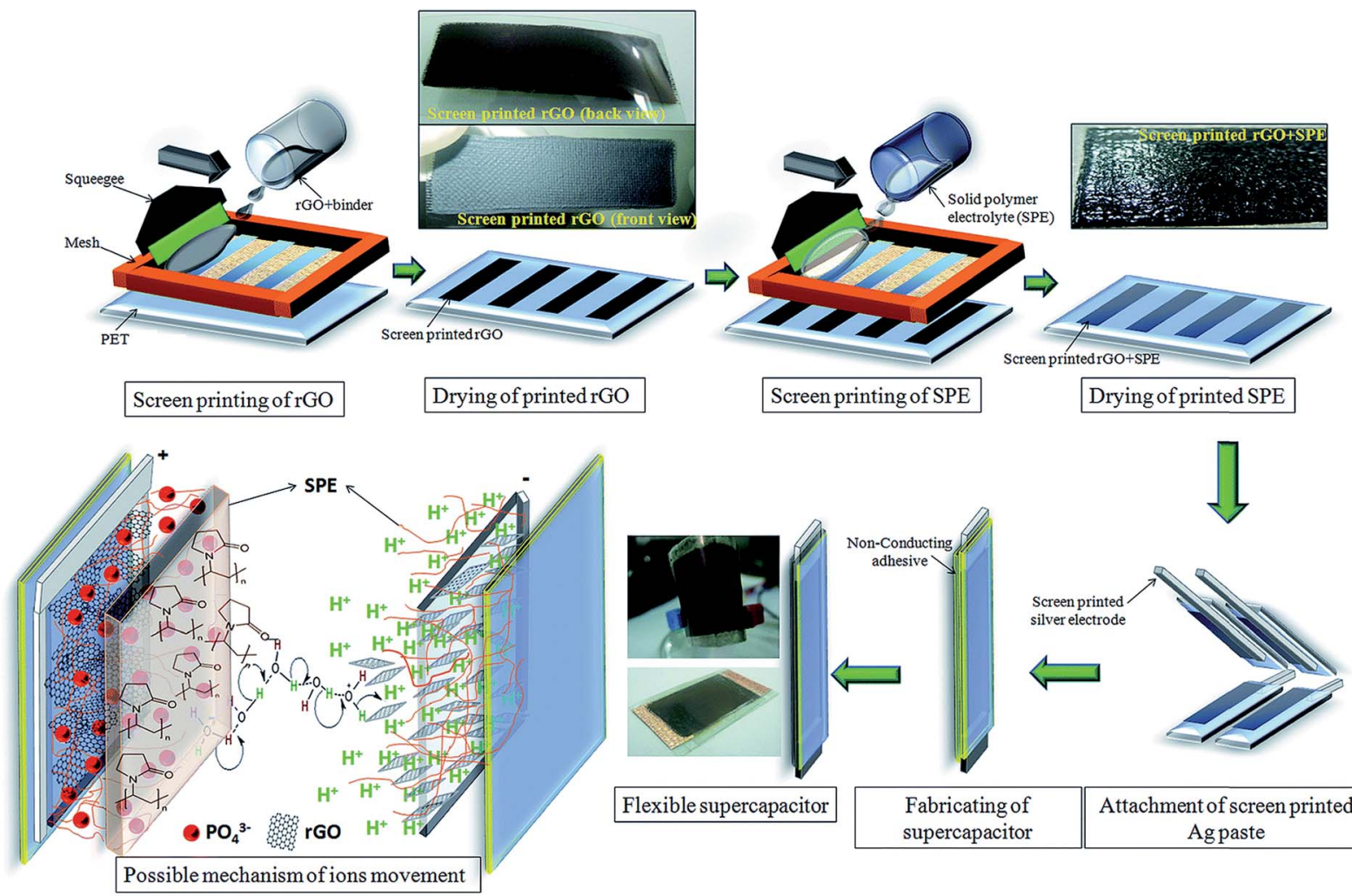

Flexible supercapacitor

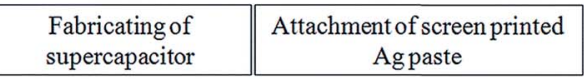

Fig. 4 Screen printing of rGO + binder and SPE on PET and fabrication of SCs with the possible mechanism of ion transportation. ${ }^{66}$ Adopted from ref. 66 with permission from The Royal Society of Chemistry. 


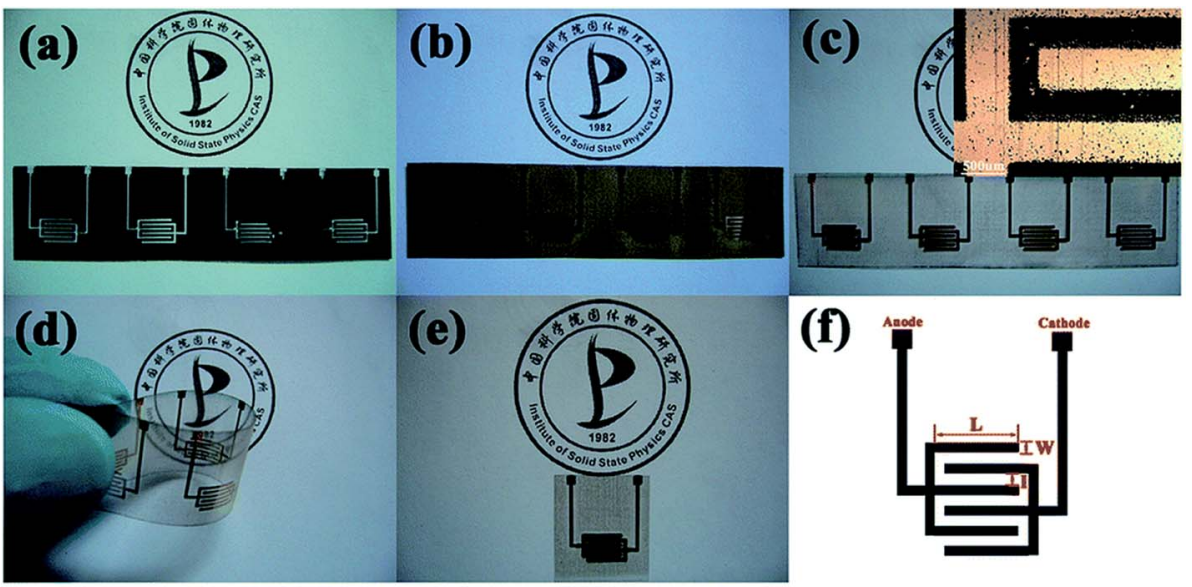

Fig. 5 Schematic illustrations of the procedure for fabricating flexible MSCs with interdigital Au/PANI network hybrid electrodes on a chip: (a) flexible PET film with the printed interdigital circuit template, (b) thin layer of Au deposited on the printed interdigital circuit template, (c) and (d) the obtained Au interdigital finger-like current collectors on the PET film after removal of the printed circuit template, (e) as-obtained interdigital Au/PANI network hybrid electrodes on a chip, (f) schematic diagram of the symmetric MSCs with 6 -interdigital fingers. The inset in (c) shows the optical microscopy image of Au interdigital-finger-like current collectors. ${ }^{70}$ Adopted from ref. 70 with permission from The Royal Society of Chemistry, 2014

cell graphite for SCs by using a solid polymer electrolyte $\left(\mathrm{H}_{3} \mathrm{PO}_{4}\right.$ doped biodegradable poly(vinylpyrrolidone) (PVP)). They used $200 \mu$ mesh and manual screen printing to fabricate rGO/PVP electrodes over a PET substrate of $3 \mathrm{~cm} \times 2 \mathrm{~cm}$ having a material loading of $3 \mathrm{mg}$. A schematic illustration of the fabrication technique along with the proposed ion transport mechanism is demonstrated in Fig. 4. SSCs made with such electrodes showed a specific capacitance of $201 \mathrm{~F} \mathrm{~g}^{-1}$ at a scan rate of $2 \mathrm{mV} \mathrm{s}^{-1}$, energy density of $11 \mathrm{~W} \mathrm{~h} \mathrm{~kg}^{-1}$ at a power density of $2 \mathrm{~kW} \mathrm{~kg}^{-1}$ and $0.2 \mathrm{~A} \mathrm{~g} \mathrm{~g}^{-1}$ current density. SSCs exhibited good cyclic stability at $0.2 \mathrm{~A} \mathrm{~g}^{-1}$ after 2000 cycles and $97 \%$ of the initial specific capacitance was retained. ${ }^{66}$

Eco-friendly commercial SCs were screen printed $\left(56 \mathrm{~cm}^{2}\right)$ over paper using activated carbon as the electrode material by Pettersson et al. ${ }^{68}$ The SCs were proposed for both large and small scale applications. They found that glyceline as the electrolyte material offers superior performances in SCs to ionic liquids such as 1-ethyl-3-methylimidazolium ethyl sulphate (EcoEng) and 1-ethyl-3-methylimidazolium bis(trifluoromethylsulfonyl)imide (EMIM:TFSI). Glyceline based SCs showed a specific capacitance of $173 \mathrm{mF} \mathrm{cm}^{-2}$ along with an energy density of $54 \mu \mathrm{W} \mathrm{h} \mathrm{cm}{ }^{-2}$ at a power density of $1614 \mu \mathrm{W}$ $\mathrm{cm}^{-2}$. Wang et al. used $\mathrm{MnO}_{2}$ /onion-like carbon as the electrode material and PVA- $\mathrm{H}_{3} \mathrm{PO}_{4}$ as the gel electrolyte for making flexible MSCs via screen printing. ${ }^{59}$ The specific capacitance of such fabricated MSCs was found to be $7.04 \mathrm{mF} \mathrm{cm}^{-2}$ at a current density of $20 \mu \mathrm{A} \mathrm{cm}^{-2}$ and up to $80 \%$ specific capacitance was retained after 1000 charge-discharge cycles. Dighe et al. fabricated screen printed ASCs (electrode size $1 \mathrm{~cm} \times 1 \mathrm{~cm}$ ) by using $\mathrm{LiCoO}_{2}$ as the positive electrode and GO as the negative electrode in $\mathrm{LiClO}_{4}$ aqueous electrolyte. ASCs showed an energy density of $19.2 \mathrm{~W} \mathrm{~h} \mathrm{~kg}^{-1}$ at a power density of $8.7 \mathrm{~kW} \mathrm{~kg}^{-1}$. The specific capacitance was found to be $59 \mathrm{~F} \mathrm{~g}^{-1}$ at a current density of $2 \mathrm{~mA} \mathrm{~cm}^{-2}$ and $85 \%$ of the initial specific capacitance was retained after 1500 cycles at a $100 \mathrm{mV} \mathrm{s}^{-1}$ scan rate. ${ }^{69}$
3.1.3 Laser printing. Laser printing is a simple, facile and rapid printing technique which offers accurate control of the inter-electrode distance up to 100 micron scale. ${ }^{70} \mathrm{~A}$ laser printer is generally a typical computer printer that uses a laser beam to draw patterns on a charged selenium-coated drum and then this drum is rolled in a toner which is a type of dry powder ink. Afterwards the ink is transferred to substrates by applying heat and pressure, then the extra ink and charge on the drum are removed. ${ }^{71}$ For instance Hu et al. used an HP LaserJet 400 M401 printer to prepare interdigitated electrodes on a flexible PET substrate and modified it with a thin layer of $\mathrm{Au}$ through thermal evaporation. ${ }^{70}$ The printed circuit template and PANI nanowire coating were removed over gold current collectors via in situ electro-polymerization. A schematic illustration of the fabrication of interdigitated electrodes by using laser printing is demonstrated in Fig. 5. The MSCs showed a high areal capacitance of $26.49 \mathrm{mF} \mathrm{cm}^{-2}$ and volumetric capacitance of $67.06 \mathrm{~F}$ $\mathrm{cm}^{-3}$ at a current density of $0.1 \mathrm{~mA} \mathrm{~cm} \mathrm{~cm}^{-3}$ along with a volu-


showed $72.7 \%$ retention after 1000 cycles and it also showed almost unchanged cycling behavior even at bending angles of up to $90^{\circ}$ at a $200 \mathrm{mV} \mathrm{s}^{-1}$ scan rate.

3.1.4 Roll-to-roll (R2R) printing technique. Roll-to-roll gravure-offset printing consists of three major processes, namely doctoring, off and set processes. Initially during the doctoring process ink is removed with the help of a doctor blade so that it can fill the engraved cell of a pattern roll. The off process involves the transfer of ink from the engraved cell to a soft blanket made up of silicone rubber by applying sufficient force, while during the set process ink is transferred to the flexible substrates in order to print the desired pattern followed by thermal drying as demonstrated in Fig. $6 .^{72}$

$\mathrm{R} 2 \mathrm{R}$ gravure printing is itself a very fast printing technique but it was further improved by Lee et al. by combining it with 


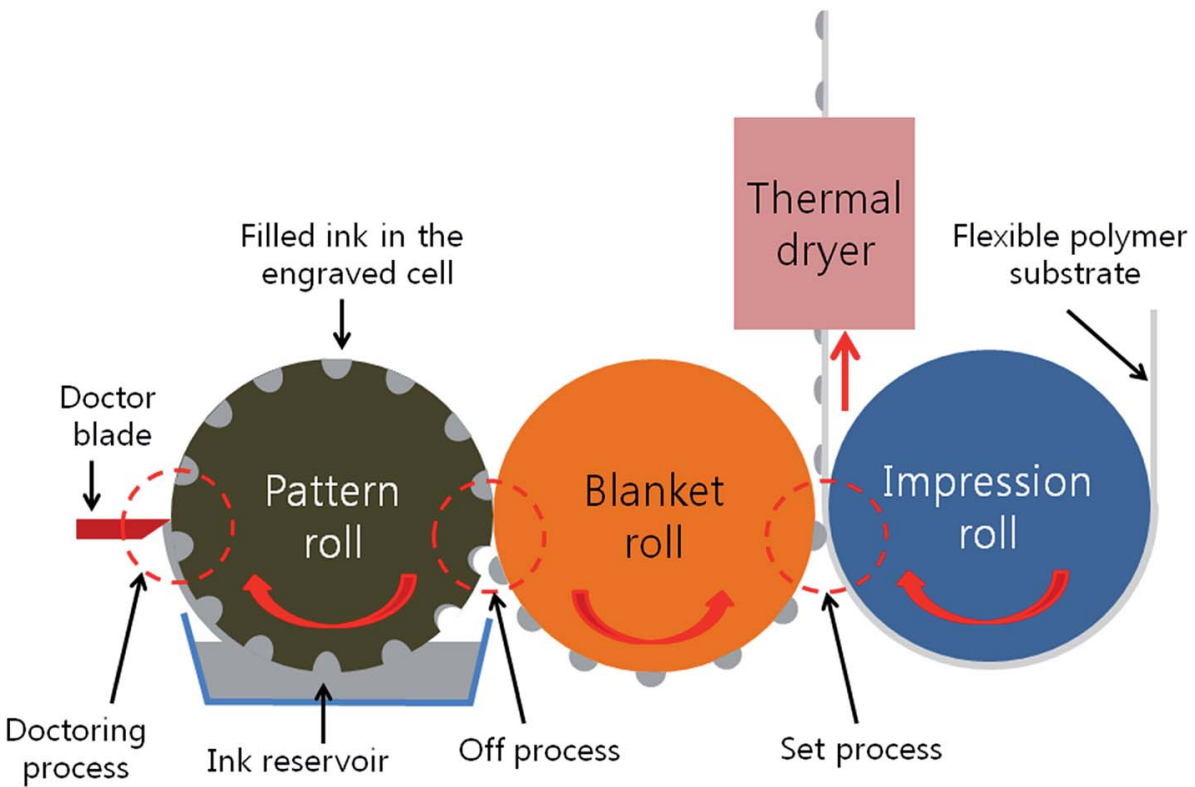

Fig. 6 Schematic of the gravure-offset printing process and printing module. ${ }^{72}$ Adopted from ref. 72 with permission from Elsevier.

(a)

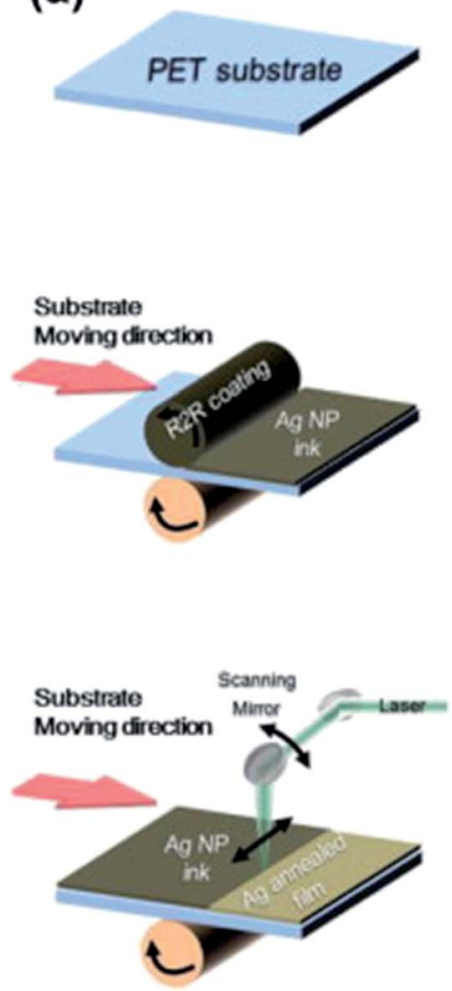

(b)

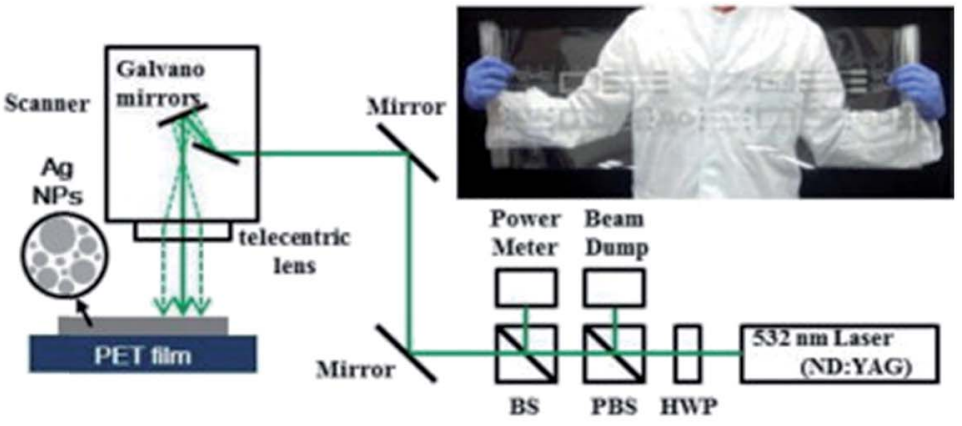

(c)
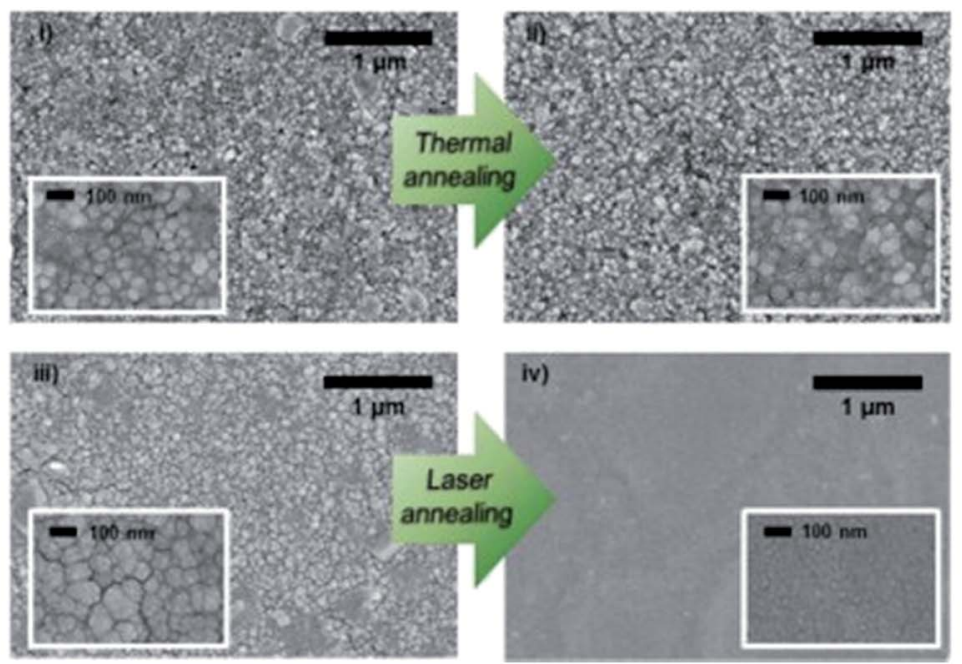

Fig. 7 (a) Schematic diagram of the entire process. Firstly, Ag nanoparticle ink is deposited on the PET film by the R2R gravure offset process and annealed by rapid scanning of the focused laser beam by using a galvano-mirror and telecentric lens. (b) Illustration of the optical experimental setup. The inset shows the digital image of the as-deposited Ag nanoparticle ink pattern on PET by the R2R process. (c) SEM images of the fabricated Ag NP film using thermal and laser annealing: ( $i$ and ii) before and after thermal annealing; (iii and iv) before and after laser annealing. Each inset shows a high magnification SEM image in each annealing process. ${ }^{72}$ Adopted from ref. 72 with permission from Elsevier. 
a laser annealing process. ${ }^{73}$ They synthesized Ag nanoparticle film (as the current collector) over a flexible PET substrate by using the R2R gravure technique. After that the Ag film was annealed with $200 \mathrm{~mW}$ laser at $1000 \mathrm{~mm} \mathrm{~s}^{-1}$ scanning speed. 1 $\mathrm{mg} \mathrm{cm}^{-2}$ of carbon was loaded over the $\mathrm{Ag}$ film via the doctor blading method to fabricate symmetric all solid state SCs in PVA- $\mathrm{H}_{3} \mathrm{PO}_{4}$ gel electrolyte. In a similar study Yeo et al. used the laser annealing technique for the synthesis of $\mathrm{Ag}$ nanoparticle film over a PET substrate. ${ }^{72}$ They showed that by altering the wavelength of the laser beam in such a way that it is absorbed by the metal nanoparticle film, it is possible to induce a confined photothermal reaction for melting the metal nanoparticle film without damaging the whole substrate. The entire process is demonstrated in Fig. 7(a). The optical experimental setup and SEM characterizations of $\mathrm{Ag}$ nanoparticles before and after annealing are shown in Fig. 7(b) and (c). $86 \%$ retention of the initial specific capacitance was observed after 1200 cycles even at various physical bending angles at a current density of $5 \mathrm{~mA}$ $\mathrm{cm}^{-2}$.

Xiao et al. used GO solution ( $5 \mathrm{mg} \mathrm{mL}^{-1}$ ) then rolled it back and forth to achieve uniform coating over commercial paper. ${ }^{44}$ The rGO/PANI/rGO freestanding flexible paper electrode was fabricated as the electrode material in $1 \mathrm{M} \mathrm{H}_{2} \mathrm{SO}_{4}$ aqueous electrolyte solution. Strong $\pi-\pi$ interaction existed between the rGO layer and PANI and the extra rGO layer over PANI/rGO paper provided a good conductive network due to the high conductivity of rGO, which further helped to increase the overall capacitance of the MSCs. SEM micrographs of top and crosssectional views of all the three types of nanohybrid papers are demonstrated in Fig. 8(a-f). Electrochemical characterization of all the three types of MSCs is shown in Fig. $8(\mathrm{~g}-\mathrm{i})$. The specific capacitance of the $\mathrm{rGO} / \mathrm{PANI} / \mathrm{rGO}$ electrode was found to be 581 $\mathrm{F} \mathrm{g}^{-1}$ at a current density of $1 \mathrm{~A} \mathrm{~g}^{-1}$ which was higher than that of $\mathrm{rGO}\left(55 \mathrm{~F} \mathrm{~g}^{-1}\right)$ and PANI/rGO $\left(522 \mathrm{~F} \mathrm{~g}^{-1}\right)$ at the same current density. The cycling was performed at a current density of $10 \mathrm{~mA}$ $\mathrm{cm}^{-2}$ which showed that the $\mathrm{rGO} / \mathrm{PANI} / \mathrm{rGO}$ electrode retained $85 \%$ of the initial specific capacitance after 10000 cycles.

In another study by Coustan et al. $\mathrm{MnO}_{2}$ film was coated over a glass plate and rolled several times to obtain thick films of $\mathrm{MnO}_{2} \cdot{ }^{74}$ The symmetric SC $(1 \mathrm{~cm} \times 1 \mathrm{~cm})$ was fabricated and tested in $1 \mathrm{M} \mathrm{Na}_{2} \mathrm{SO}_{4}$ electrolyte at a potential window of $0-0.85$ $\mathrm{V}$. It was reported that $\mathrm{MnO}_{2}$ in the presence of sodium dodecyl sulfate (SDS) gave a BET surface area of $273 \mathrm{~m}^{2} \mathrm{~g}^{-1}$ and specific capacitance of $164 \mathrm{~F} \mathrm{~g}^{-1}$ at a scan rate of $10 \mathrm{mV} \mathrm{s}^{-1}$, higher than those obtained without SDS $\left(133 \mathrm{~F} \mathrm{~g}^{-1}\right.$, BET surface area $78 \mathrm{~m}^{2}$ $\left.\mathrm{g}^{-1}\right)$ at the same current density.

Qui et al. used the imprinting technique to prepare a 3D $\mathrm{MnO}_{x} / \mathrm{Au}$ nanocone array electrode for pseudocapacitors $(1 \mathrm{~cm}$ $\times 1 \mathrm{~cm}) .{ }^{75}$ The nanocone arrays at different growth stages are shown in Fig. 9. The specific capacitance for the $\mathrm{MnO}_{x} / \mathrm{Au}$ nanocone electrode in a three electrode system using $1 \mathrm{M}$ $\mathrm{Na}_{2} \mathrm{SO}_{4}$ electrolyte was found to be $840.3 \mathrm{~F} \mathrm{~g}^{-1}$ at a current density of $2 \mathrm{~A} \mathrm{~g}^{-1}$. The pseudocapacitor based on the $\mathrm{MnO}_{x} / \mathrm{Au}$ nanocone as the positive electrode and carbon as the negative
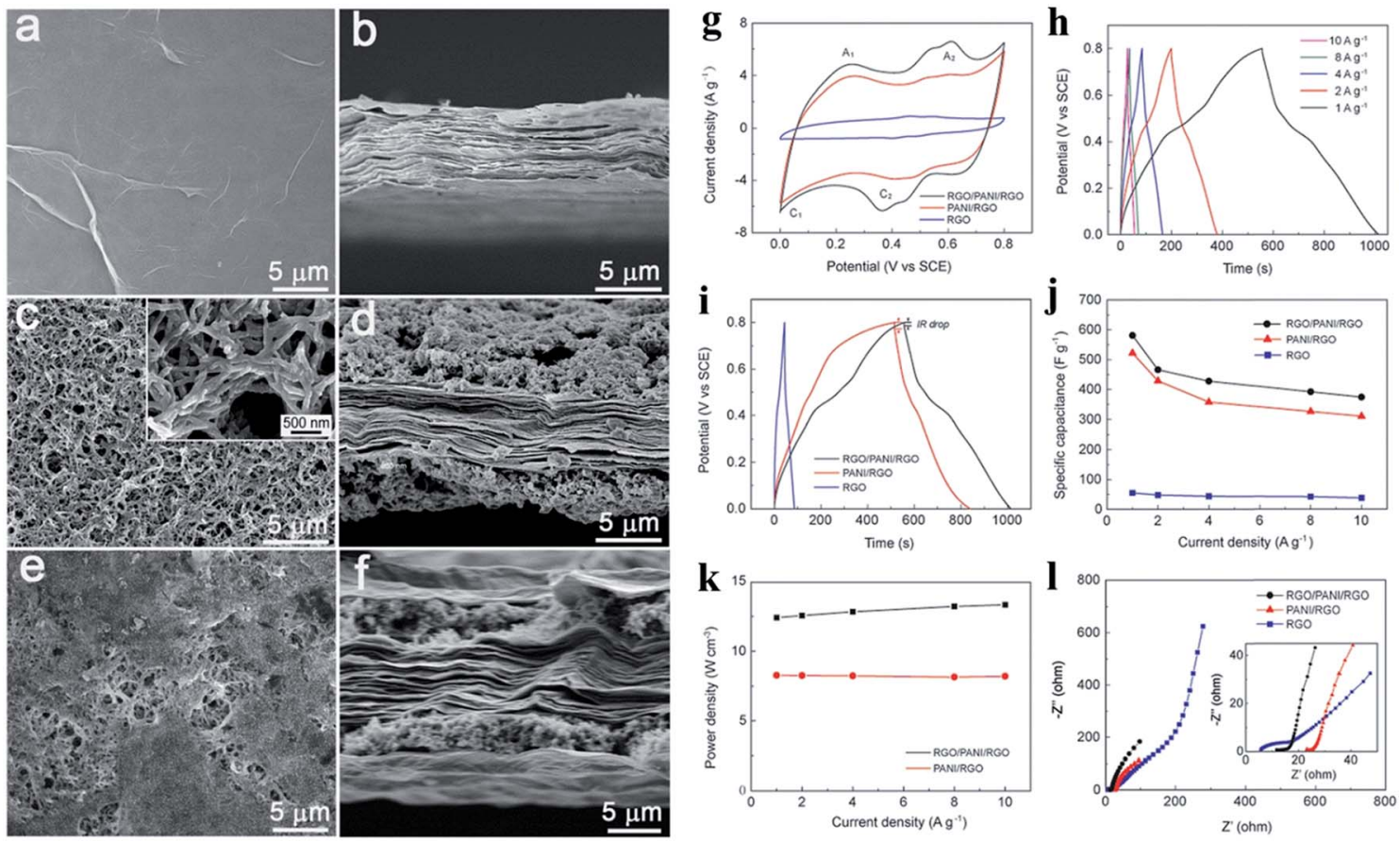

Fig. 8 Top view and cross-sectional view SEM images of (a and b) rGO paper, (c and d) PANI/rGO paper, (e and f) rGO/PANI/rGO paper, (g) CV curves of $\mathrm{rGO} / \mathrm{PANI} / \mathrm{rGO}, \mathrm{PANI} / \mathrm{rGO}$ and $\mathrm{rGO}$ paper electrodes at a scan rate of $10 \mathrm{mV} \mathrm{s}^{-1}$ in $1 \mathrm{M} \mathrm{H}_{2} \mathrm{SO}_{4}$ electrolyte, (h) GCD curves of rGO/PANI/ $\mathrm{rGO}$ paper at different current densities, (i) GCD curves of $\mathrm{rGO} / \mathrm{PANI} / \mathrm{rGO}, \mathrm{PANI} / \mathrm{rGO}$ and $\mathrm{rGO}$ paper electrodes at a current density of $1 \mathrm{~A} \mathrm{~g}^{-1}$, (j) specific capacitances of $\mathrm{rGO} / \mathrm{PANI} / \mathrm{rGO}, \mathrm{PANI} / \mathrm{rGO}$ and $\mathrm{rGO}$ paper electrodes versus discharge current densities, (k) power densities of rGO/ $\mathrm{PANI} / \mathrm{rGO}$ and PANI/rGO paper electrodes versus discharge current densities, (l) Nyquist plots of rGO/PANI/rGO, PANI/rGO and rGO paper electrodes. ${ }^{44}$ Adopted and modified from ref. 44 with permission from Nature Publishing Group, 2015. 

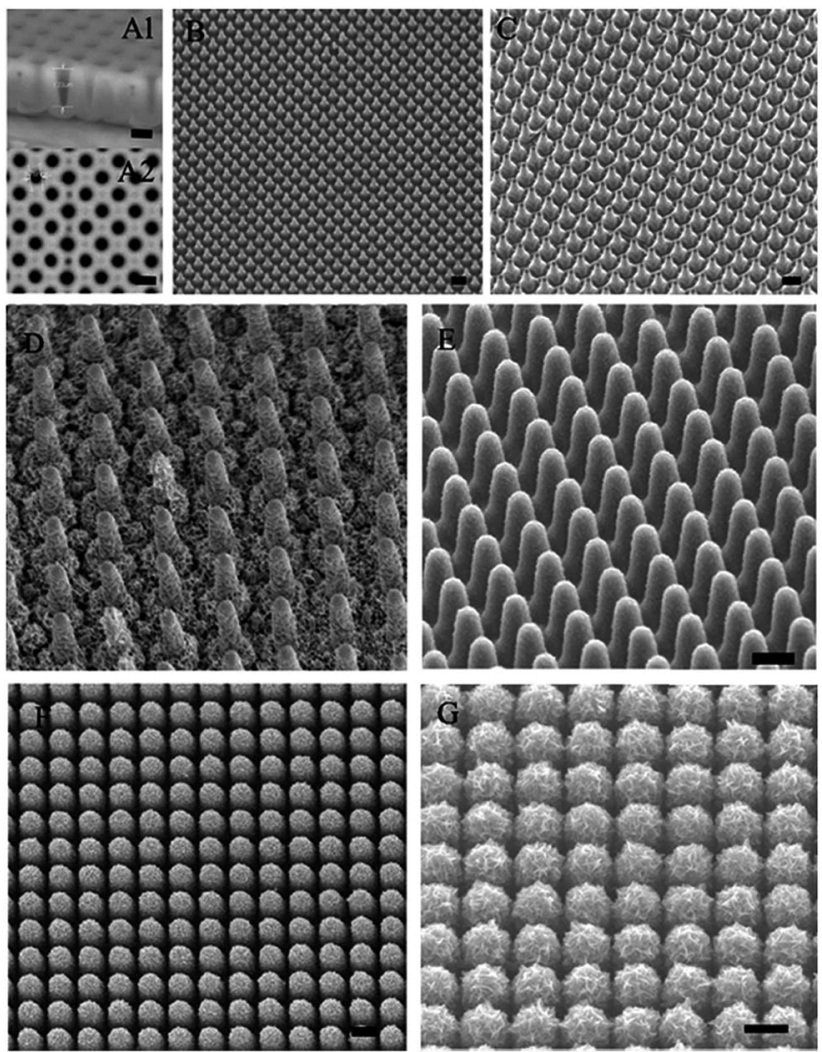

Fig. 9 Microscopic characterizations of the 3D nanocone arrays at different stages of the pattern transfer and outer layer deposition: (a) cross-sectional $\left(A_{1}\right)$ and top $\left(A_{2}\right)$ SEM images of an as-prepared nanocone array mold with a height of $\sim 1.7 \mu \mathrm{m}$ and an open-diameter of $\sim 580 \mathrm{~nm}$, (b) SEM image of a desired PTPE nanocone array obtained from mold $A$, (c) a metal nanocone array electrode obtained by magnetron sputtering of the $\mathrm{Au}$ metal, $(\mathrm{D}-\mathrm{G})$ electrodeposition of $\mathrm{MnO}_{x}$ on the Au metal nanocone array electrode at different deposition times of 70 s, 175 s, 350 s and 700 s, respectively. All scale bars: 1 $\mu \mathrm{m} .{ }^{75}$ Adopted from ref. 75 with permission from The Royal Society of Chemistry.

electrode achieved a specific capacitance of $108.5 \mathrm{~F} \mathrm{~g}^{-1}$ at a current density of $1 \mathrm{~A} \mathrm{~g}^{-1}$ at $1.8 \mathrm{~V}$ operating potential. The corresponding energy density was found to be $46.8 \mathrm{~W} \mathrm{~h} \mathrm{~kg}^{-1}$ at a power density of $0.72 \mathrm{~kW} \mathrm{~kg}^{-1}$ and $96.5 \%$ of the initial capacitance was retained after 2000 cycles at $2 \mathrm{~A} \mathrm{~g}^{-1}$.

3.1.5 Micro-extrusion printing. Micro-extrusion technique is a high throughput printing technique which provides programmable and controllable printing for both $2 \mathrm{D}$ and $3 \mathrm{D}$ architectures. It also enables printing on different substrates with various printing materials. Sun et al. have introduced the micro-extrusion printing technique for the fabrication of a flexible rGO electrode for planar MSCs. ${ }^{76}$ In the mirco-extrusion technique, viscous ink is passed through a nozzle with controlled speed and pre-determined trajectory. The motion of the nozzle is controlled with a desktop XYZ motor and a high precision displacement pump. As-prepared interdigitated SCs with $\mathrm{PVA}-\mathrm{H}_{2} \mathrm{SO}_{4}$ gel electrolyte showed a volumetric specific capacitance of $56.5 \mathrm{~F} \mathrm{~cm}^{-3}$ at a current density of $0.06 \mathrm{~A} \mathrm{~cm}^{-3}$.

3.1.6 Selective laser melting printing technique. Zhao et al. adapted an advanced selective laser melting (SLM) technology for the fabrication of $3 \mathrm{D}$ titanium interdigitated electrodes in $\mathrm{SC}$ applications with a solid gel electrolyte $\left(\mathrm{PVA}-\mathrm{H}_{3} \mathrm{PO}_{4}\right) \cdot{ }^{57}$ In the SLM technology they used a Realizer SLM50 metal printer to produce a high-power laser beam and focused it on a metal bed of fine metallic powder. The laser selectively fused the metal according to the pre-determined software program. 3D Ti electrodes were further modified with PPy through electropolymerization. The fabricated MSCs with these electrodes are shown in Fig. 10. The as-assembled solid-state SSCs achieved a volumetric capacitance of $2.4 \mathrm{~F} \mathrm{~cm}^{-3}$ at a current density of $3.74 \mathrm{~mA} \mathrm{~cm}^{-3}$ and the corresponding volumetric energy density was found to be $213.5 \mathrm{~W} \mathrm{~h} \mathrm{~m}^{-3}$.

\subsection{Lithography techniques}

Fabrication of carbon nanoparticles (CNPs) with desired nanoarchitectures is usually achieved by a template assisted technique via diffusion of a carbon precursor into an inorganic template. However, the large scale synthesis of CNPs via the template assisted technique is limited. Recently nanoimprinting lithography has been introduced as an economic alternative to print CNPs at a gram scale from thermoplastic polymers. ${ }^{77}$ But it requires harsh synthesis conditions such as high temperature, pressure as well as requirement of a highly viscous precursor. A simple, fast and low cost, spin-on nanoprinting (SNAP) technique came into focus for the fabrication of CNPs as the electrode material with a high surface area. This technique basically involved the spin-on of a thin layer of solution into a prefabricated mold and then substrate transfer. Thomas and co-workers ${ }^{78}$ adapted SNAP lithography techniques for the fabrication of a carbon nanostructure that was further applied as an electrode material in SCs. A schematic representation of this technique is shown in Fig. 11.

Deposition and patterning of electrode materials are significant for the miniaturization of SCs. Among the generally used nanocarbons, photoresist-derived pyrolyzed carbon is one of the widely used electrode materials in energy storage devices due to its excellent electrical properties and ease of synthesis. ${ }^{40}$ Wang et al. investigated a simple and scalable technique for the fabrication of high-performance MSCs by on-chip integration. ${ }^{79}$ On-chip integration was done by combining micro-fabrication and sol-gel approach. An interdigitated electrode of photoresist-derived pyrolyzed carbon was patterned on planar silica wafers via UV lithography. An ionogel electrolyte was then transferred to the interdigitated electrode and was separated by physical separation. The fabrication technique is demonstrated in Fig. 12(a) and optical characterizations are shown in Fig. 12(b-d). The initial capacitance remained intact even after 10000 cycles, confirming the long cycle life of the MSCs. The authors observed a maximum energy density of $\sim 3 \mathrm{~mW} \mathrm{~h} \mathrm{~cm}^{-3}$; more significantly it was higher than those of thin film commercial Li-ion batteries and the maximum achieved power density was reported to be $26 \mathrm{~W} \mathrm{~cm}^{-3}$. The Nyquist plot and Bode plots are shown in Fig. 12(e) and (f).

Hierarchical carbon nanostructures hold great potential in energy storage devices due to their large surface area and tunable mechanical compliance. ${ }^{\mathbf{0}}$ Photolithography in 


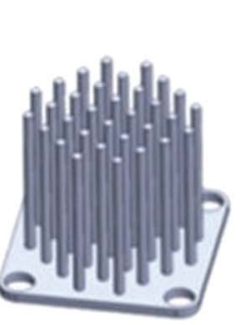

Top electrode

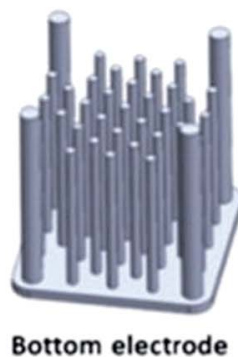

Electrodeposition

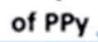

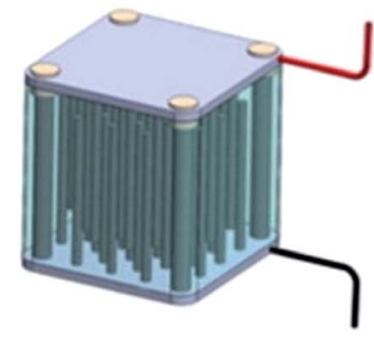
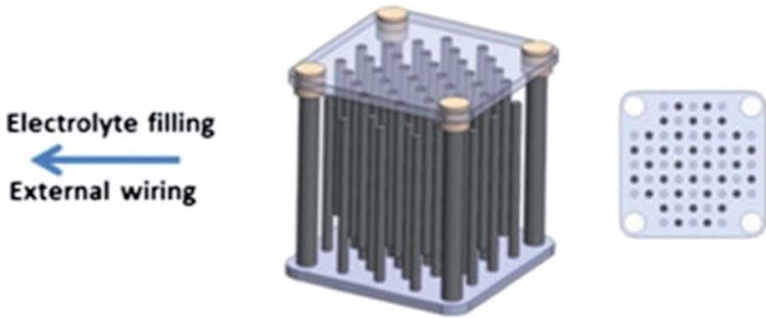

Fig. 10 Schematic procedures used to fabricate solid state SCs, including the design of interdigitated Ti electrodes, PPy electropolymerization and device assembly. ${ }^{57}$ Adopted from ref. 57 with permission from Elsevier.

combination with an ion etching process is a widely used technique for the fabrication of hierarchical structures. ${ }^{81}$ However till date, only few reports are available for the fabrication of hierarchical carbon nanostructures with lithographic techniques. ${ }^{81-83}$ Jiang et al. proposed a facile, economic technique for the large scale fabrication of hierarchical carbon arrays by utilizing nanoimprint lithography followed by oxygen plasma etching on both micro and nano levels. ${ }^{84}$ The fabrication technique basically involves three steps: fabrication of hierarchical carbon arrays on a nano/microlevel with nanoimprint lithography followed by oxygen plasma etching and finally pyrolysis and further electrodeposition of $\mathrm{MnO}_{2}$ film on

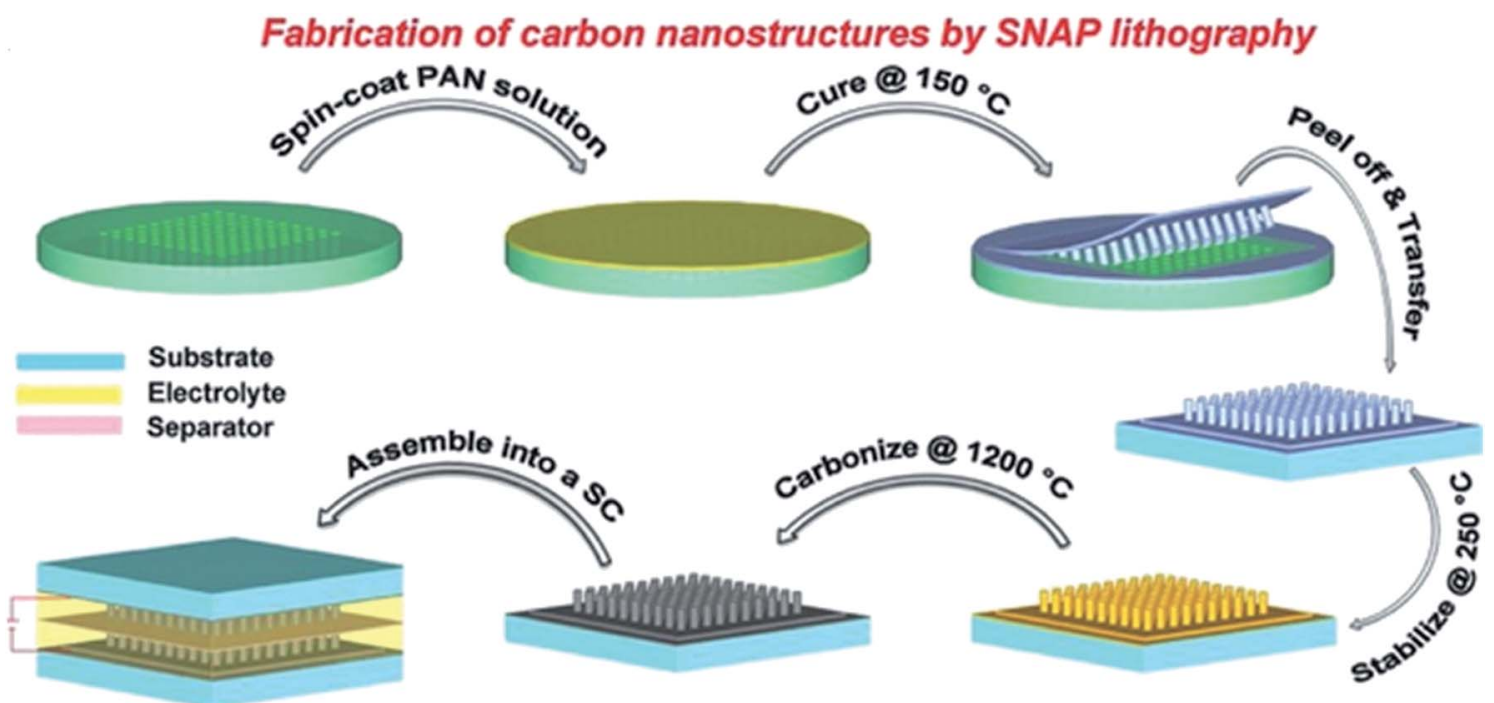

Fig. 11 Fabrication of carbon nanostructures by the SNAP method. A pre-fabricated mold is spin coated with a partially cyclized polyacrylonitrile (PAN) solution. The film is cured at $150^{\circ} \mathrm{C}$ before transferring it to a substrate coated with pre-cured PAN film. A nanostructured PAN film is obtained by separating the mold. The PAN film is stabilized by heating to $250^{\circ} \mathrm{C}$ for $3 \mathrm{~h}$. This film is heat treated at higher temperatures to convert it to carbon/graphite. An EDLC SC device is fabricated with two nanostructured carbon electrodes. ${ }^{78}$ Adopted from ref. 78 with permission from WILEY-VCH Verlag GmbH \& Co. KGaA, Weinheim. 


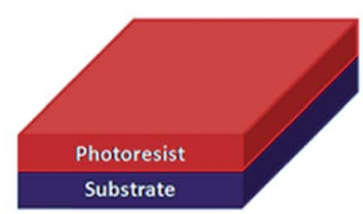

(a)

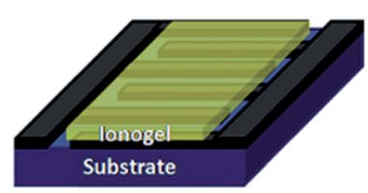

(e)

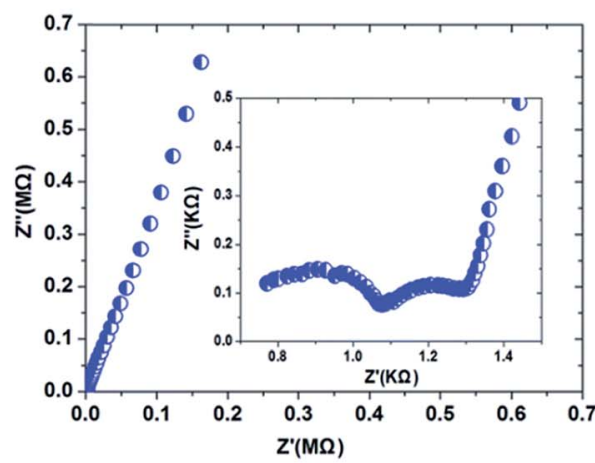

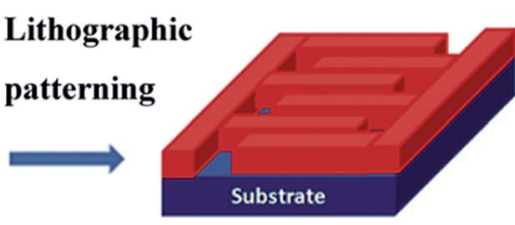

Pyrolysis

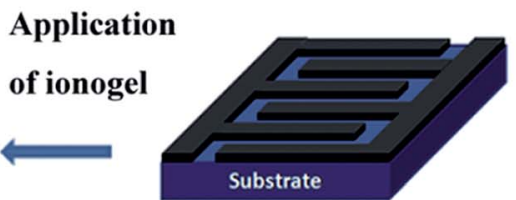

(f)

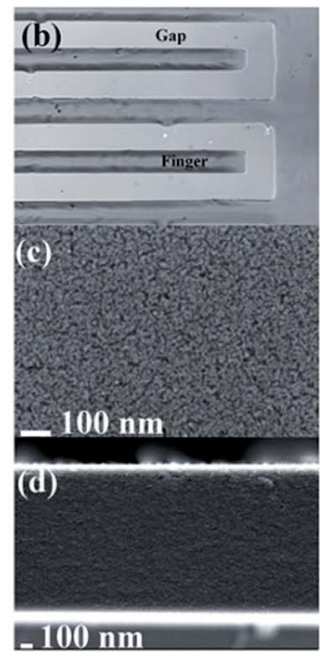

nm

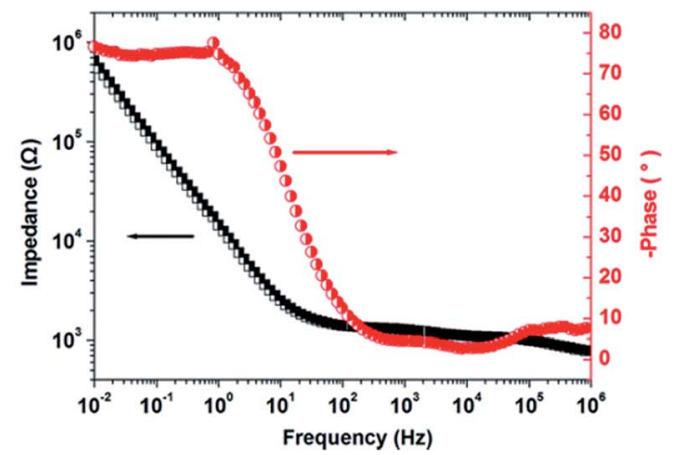

Fig. 12 (a) Schematic illustration of a micro-supercapacitor device fabrication process. (b) Optical image of the patterned photoresist-derived porous carbon lines after pyrolysis. (c) SEM top-view and (d) SEM cross-sectional view of a pyrolyzed line. AC impedance data showing the (e) Nyquist plot with an enlarged view of the high-frequency region provided in the inset and (f) Bode plots. ${ }^{79}$ Adopted and modified from ref. 79 with permission from The Royal Society of Chemistry, 2014.

the carbon array. The hierarchical morphology restricted the collapse of the carbon nanostructures and in addition provided good electrical connections and shortened the ion diffusion path. Song et al. summarized the fabrication of MSCs based on the carbon micro-electromechanical system (C-MEMS) techniques with different architectures and active electrode materials. ${ }^{85}$ The term C-MEMS was defined for a set of fabrication methods (stamping, machining, casting and lithography) and was used for the fabrication of glassy nanocarbons by using a pre-patterned photoresist polymer as a precursor. This technique was based on the pyrolysis of a patterned polymer photoresist and is promising for the fabrication of $2 \mathrm{D}$ and $3 \mathrm{D}$ electrodes for microscale energy storage devices.

\section{Electrolytes for interdigitated electrodes in micro-supercapacitors}

The amount of energy stored in the supercapacitors and how quickly this stored energy can be released will depend on the type of electrolyte used in the supercapacitors. ${ }^{86}$ Ionic liquids and their derivatives are widely used in energy storage devices owing to their interesting properties such as high thermal stability, wide operating potential window $(>4 \mathrm{~V})$, low vapor pressure and non-flammability. ${ }^{87,88}$ The operating voltage window is $1.229 \mathrm{~V}$ for aqueous electrolytes, which corresponds to the thermodynamic decomposition of water. Organic electrolytes can go up to $2.7 \mathrm{~V}$ (for propylene carbonate solvent) and ionic liquid electrolytes can go up to $6 \mathrm{~V}$, typically about $4.5 \mathrm{~V}$ and store a large amount of energy. ${ }^{8,23,86}$ The conductivities of aqueous electrolytes are the highest and hence the charge transfer is faster. $\mathrm{H}_{2} \mathrm{SO}_{4}, \mathrm{KOH}, \mathrm{NaCl}, \mathrm{Na}_{2} \mathrm{SO}_{4}$ and $\mathrm{KCl}$ are widely used as aqueous electrolytes. Tetraethylammonium tetrafluoroborate, tetraethylphosphonium tetrafluoroborate and triethylmethylammonium tetrafluoroborate along with their $\mathrm{PF}_{6}{ }^{-}$salts in propylene carbonate in acetonitrile solvent also find their applications as electrolytes. The mainly used ionic electrolytes include imidazolium, pyrrolidinium and asymmetric aliphatic quaternary ammonium salts with trifluoromethanesulfonate, tetrafluoroborate, bis(fluorosulfonyl) imide, bis(trifluoromethanesulfonyl)imide, and hexafluorophosphate as anions..$^{12,23,86}$

Heat sealed SCs with aqueous electrolytes $\left(\mathrm{K}_{2} \mathrm{SO}_{4}\right.$ and $\left.\mathrm{H}_{2} \mathrm{SO}_{4}\right)$ face the serious issue of solvent evaporation. To overcome this issue Yanfei et al. used ionic liquid electrolytes with a low 
vapour pressure such as 1-butyl-3-methylimidazolium tetrafluoroborate $\left(\mathrm{BMIM} \mathrm{BF}_{4}\right){ }^{58}$ In general, voids and defects present in the crystal lattice of solid electrolytes used in MSCs are responsible for electricity conduction. Leakage during encapsulation of liquid electrolytes can be overcome by using solid electrolytes, as solid electrolytes are dispersed and bound in a polymeric matrix. ${ }^{7} \mathrm{PVA} / \mathrm{H}_{3} \mathrm{PO}_{4}, \mathrm{PVA} / \mathrm{H}_{2} \mathrm{SO}_{4}, \mathrm{PVA} / \mathrm{KOH}, \mathrm{PVA} /$ $\mathrm{NaNO}_{3}, \quad \mathrm{PVA} / \mathrm{LiCl}$, polybenzimidazole $/ \mathrm{H}_{3} \mathrm{PO}_{4}$, polyethyleneoxide $/ \mathrm{LiClO}_{4}$, and 1-ethyl-3-methylimidazolium bis(trifluoromethylsulfonyl)imide are few examples of solid electrolytes used in macro- and micro-supercapacitors., ${ }^{7,12}$

\section{Materials for interdigitated electrodes in micro-supercapacitors}

Over the past few decades availability of cost-effective synthesis techniques for advanced nanostructured materials has facilitated their potential applications in energy storage devices. Challenges and opportunities towards new and existing nanomaterials have become a fast growing research field. However, till date synthesis of suitable electrode materials for fabricating interdigitated electrodes in MSCs with robust performance and stability is still a major challenge.

The flourish of nanoscience and nanotechnology brings to the modern society a mysterious verve of multifunctional nanomaterials along with their various potential applications in diverse fields. The nanomaterials render novel functions and properties for advanced potential energy storage applications, which are promising building blocks for novel products and unique properties. In order to fabricate interdigitated electrodes, carbon nanomaterials, conductive polymers, metal oxides and hybrid nanomaterials have been used as active materials. $^{3}$

\subsection{Carbon nanomaterials based interdigitated electrodes}

The discovery of advanced nanostructured carbons brings significant improvement in technological applications for the exploration of new materials in micro-scale energy storage devices. ${ }^{3,89}$ Porous activated, ${ }^{82,90}$ templated and carbide-derived carbons, ${ }^{27}$ multi- and single-walled carbon nanotubes, ${ }^{91}$ carbon aerogels, ${ }^{92}$ carbon nano-onions, ${ }^{93}$ graphene oxide ${ }^{94}$ and multilayer graphene ${ }^{26}$ are widely used as electrode materials in MSCs. ${ }^{4}$ Morphological variations of CNPs provide a range of potential technological applications due to their unique optical, electronic, physiochemical properties, chemical versatility and ease-to-manipulate surface properties. ${ }^{95}$ Structural variations such as aspect ratio, porosity, hybridization (arising from the crystal structure and lattice packing) play a significant role in their performance in energy storage devices. ${ }^{89,95}$ The high aspect ratio of porous CNPs significantly reduces the diffusion time with excellent stability in MSCs. ${ }^{89}$ Consequently, there has been emerging interest in the use of CNPs for MSCs due to their maintenance-free nature and excellent life cycle. However, fabrication of CNPs with desired properties (morphology, aspect ratio, electrical conductivity and chemical/thermal stability) requires tremendous processing factors and optimization. Simultaneously, CNPs and their nano-composites with metal oxides or conductive polymers are potential candidates as emerging materials in MSCs originating from basic scientific research..$^{\mathbf{4} 96-98}$

To date carbon nanotubes (CNTs), graphene and their related nano-structures have been widely explored in the area of energy storage..$^{7,93,99,100}$ CNTs are basically of two types, singlewall (SWCNTs) and multi-wall carbon nanotubes (MWCNTs), which are semiconducting or conductive ${ }^{101,102}$ in nature depending on the rolled-up graphene sheets. Entangled networks of CNTs have also been explored for the fabrication of flexible electrodes. ${ }^{\mathbf{1 0 3}}$

It has been reported that the vertically aligned morphology of CNTs enhances the performances of MSCs because of the availability of a better path for diffusion of electrolyte ions into the depth of the electrode material. ${ }^{104}$ But vertically aligned CNTs generally exhibit poor conductivity. To overcome this, various research groups either doped them with graphene, ${ }^{\mathbf{1 0 5}}$ conducting polymers or directly grew them on a catalyst surface. ${ }^{\mathbf{1 0 6}}$ For example, Hsia et al. introduced conductivity in vertically aligned CNTs (synthesized by the CVD technique on a silicon substrate) by sputtering a Ni layer on top. ${ }^{107}$ A maskless laser-assisted dry transfer technique was used to fabricate flexible MSCs based on vertically aligned CNTs as the electrode material and ionogel as the electrolyte on a polycarbonate substrate. The specific capacitance of such MSCs was measured as $430 \mu \mathrm{F} \mathrm{cm}^{-2}$ at a scan rate of $0.1 \mathrm{~V} \mathrm{~s}^{-1}$. More significantly, upon bending negligible loss in specific capacitance was observed. Vertically aligned CNTs were also fabricated by Saleem et al. by using spin-coated polymer-stabilized palladium nanoparticles as the nanocatalyst and further utilized these in SCs as an electrode material. ${ }^{108}$ Sammoura et al. also fabricated nano-composites of PPy with vertically aligned CNTs and integrated them on silicon based electrodes for SC applications. ${ }^{109}$

The direct use of CNTs in inkjet printing is still limited due to their hydrophobic nature and hence the tendency to aggregate even at low concentrations. Applications of graphene as an electrode material are robust in contrast to CNTs due to strong inter-sheet van der Waals attractions. More accessible surface area, porosity and good electrical conductivity are the key properties of the requisite material for efficient charging of EDLCs. ${ }^{110}$ In this regard, graphene has emerged as a promising electrode material for the fabrication of planar MSCs due to its unique electrical and chemical properties as well as extremely high surface area and porous nature. ${ }^{7}$ Graphene offers an outstanding intrinsic capacitance of $21 \mu \mathrm{F} \mathrm{cm} \mathrm{cm}^{-2}$ or theoretical capacitance as high as $550 \mathrm{~F} \mathrm{~g}^{-1} \cdot \cdot^{37,111}$ Among graphene based nanomaterials rGO has been most widely used as an electrode material due to its economic nature, easily scalable and wetchemical approach to graphene fabrication. ${ }^{\mathbf{1 1 0 , 1 1 2}}$ Hence, GO based MSCs can be easily industrialized but the requirement of room/low operating temperature is a major drawback for their stability and real world applications. Wu et al. reviewed the current developmental trends for graphene based planer MSCs and their on-chip integration in energy harvesting devices. ${ }^{26}$

It is reported that device geometry and architecture significantly affect the performance of MSCs. The in-plane geometry 
along with high conductivity enhances the performance of graphene based thin film MSCs. Planar and interdigitated geometries have the benefit of confinement and transportation of electrolyte ions due to the short ion diffusion distance and hence offer high power density. Laser irradiation is the most commonly used technique for the patterning of electrodes and it was observed that by varying the laser intensity conductivity can be increased up to 5 orders of magnitude. ${ }^{113,114}$ Apart from this, ultrahigh rate planar solid state MSCs based on interdigitated finger-like electrode architecture have been fabricated by using the photolithographic micro-fabrication technique. ${ }^{32}$ An interesting approach for the fabrication of asymmetric graphene fiber based MSCs with two different types of electrodes that can simultaneously exhibit flexibility and increased energy density was reported by Gao and co-workers. ${ }^{115} \mathrm{Wu}$ et al. used the micro-patterning technique for the fabrication of in-plane interdigitated MSCs on both rigid and flexible surfaces, based on nanoscale thick graphene film. ${ }^{26}$ Fabrication of the electrode was carried out by the well-established lithographic technique for patterning graphene based interdigital microelectrodes and further oxidative etching in oxygen plasma. This graphene based in-plane MSC typically exhibited a high power density of $495 \mathrm{~W} \mathrm{~cm}^{-3}$ and a high energy density of $2.5 \mathrm{~mW} \mathrm{~h} \mathrm{~cm}^{-3}$ due to the high electrical conductivity. The fabrication process of the electrode on silicon wafer (rigid) and copper foil (flexible) with their microelectrode characterization is illustrated in Fig. 13. $\mathrm{CH}_{4}$ plasma reduction technique increases the electrical conductivity of GO (345 $\left.\mathrm{S} \mathrm{cm}^{-1}\right)$ termed as MPG, by providing an additional carbon source to repair the GO surface and hence increase the $\mathrm{C} / \mathrm{O}$ ratio. The comparison of energy and power densities of MPG-MSCs with TG-MSCs, MPG-SSCs, commercially applied electrolytic capacitors, lithium thin-film batteries, Panasonic Li-ion battery and conventional SCs demonstrated that MPG-MSCs exhibit exceptional electrochemical energy storage with simultaneous ultrahigh energy density and power density.

In another report $\mathrm{Wu}$ et al. demonstrated the fabrication of graphene based miniaturized solid state planar and interdigitated SCs with the advantage of ultrahigh rate. ${ }^{32}$ Methane plasma-assisted reduction was used for the synthesis of rGO and the photolithographic fabrication technique was adopted for the fabrication of an interdigitated electrode on silicon wafer. They explored the effect of number and area of interdigital fingers on the electrochemical performance of MSCs and came up with the conclusion that by increasing the number of fingers and narrowing the width, power/energy density, capacitance and cycle life can be enhanced. More number of fingers with a small width in the same area significantly reduce the average path of ionic diffusion and hence this reduces the resistance of the electrolyte due to the low ion transport ${ }^{\mathbf{1 1 6}}$ and also enhances the interface between the electrolyte and active material. The areal and stack capacitances of rGO based MSCs with 8, 16 and 32 interdigitated fingers were calculated to be $\sim 108 \mu \mathrm{F} \mathrm{cm} \mathrm{cm}^{-2}$ and $\sim 24.0 \mathrm{~F} \mathrm{~cm}^{-3}, \sim 111 \mu \mathrm{F} \mathrm{cm} \mathrm{cm}^{-2}$ and $\sim 24.7 \mathrm{~F}$ $\mathrm{cm}^{-3}$, and $\sim 116 \mu \mathrm{F} \mathrm{cm}^{-2}$ and $\sim 25.9 \mathrm{~F} \mathrm{~cm}^{-3}$, respectively. The energy/power density of MSCs with 32 fingers is much higher $\left(\sim 3.6 \mathrm{~mW} \mathrm{~h} \mathrm{~cm} \mathrm{~m}^{-3} / 1270 \mathrm{~W} \mathrm{~cm}^{-3}\right)$ than those of MSCs with 16 $\left(\sim 3.4 \mathrm{~mW} \mathrm{~h} \mathrm{~cm}^{-3} / 140 \mathrm{~W} \mathrm{~cm}^{-3}\right)$ and $8\left(\sim 3.3 \mathrm{~mW} \mathrm{~h} \mathrm{~cm}^{-3} / 30 \mathrm{~W}\right.$ $\mathrm{cm}^{-3}$ ) fingers.

Recently, many studies have been devoted to the enhancement of the performance of MSCs via doping of heteroatoms in graphene. Müllen and co-workers fabricated N and B doped, highly uniform and thick, large surface area graphene by using the layer-by-layer (LBL) technique for application in MSCs. ${ }^{117}$ Thin film of $\mathrm{N}$ and $\mathrm{B}$ doped graphene nanosheets was fabricated by using GO nanosheets, poly-L-lysine (PLL) and $\mathrm{H}_{3} \mathrm{BO}_{3}$. MSCs were fabricated by the lithographic technique followed by dry etching and exhibited an ultrahigh volumetric capacitance of $\sim 488 \mathrm{~F} \mathrm{~cm}^{-3}$ at a scan rate of $200 \mathrm{~V} \mathrm{~s}^{-1}$.

Immense interest and efforts were taken to explore economic and green materials for electrochemical energy storage devices due to ecological concerns and search for alternative energy sources as well. In this regard, carbon nanomaterials with interesting electrochemical characteristics are derived from a variety of green sources/waste materials in an environmentally friendly way. ${ }^{\mathbf{1 1 8 - 1 2 2}}$ Madhu et al. synthesized activated carbon decorated with CNPs exhibiting a high surface area of $1555 \mathrm{~m}^{2}$ $\mathrm{g}^{-1}$ via a green method by using dead mango leaves as the carbon precursor. This activated carbon was further applied as an electrode material for energy storage application and exhibited a specific capacitance of $55 \mathrm{~F} \mathrm{~g} \mathrm{~g}^{-1}$ and energy density of $10.75 \mathrm{~W} \mathrm{~h} \mathrm{~kg}^{-1} \cdot{ }^{90}$ In another report by Peng et al. five types of waste tea-leaves were used for the synthesis of activated carbon and further applied in the fabrication of high performance SCs. ${ }^{123}$ Sahu et al. used silk cocoon membranes for the synthesis of heavily nitrogen doped graphene. ${ }^{\mathbf{1 2 4}}$ Nitrogen doping enhances the electrical conductivity of graphene and hence the performance of SCs due to the generation of dipoles on the graphene surface. Mitlin and co-workers synthesized mesoporous interconnected partially graphitic carbon nanosheets with a high specific surface area $\left(2287 \mathrm{~m}^{2} \mathrm{~g}^{-1}\right)$ by using hemp as the bio-precursor via the combination of carbonization, activation with $\mathrm{KOH}$ and hydrothermal techniques. ${ }^{125}$ Carbon nanosheets exhibited a very high power density of 20-100 kW $\mathrm{kg}^{-1}$ depending upon the synthesis conditions, when applied as an electrode material in ionic liquids from the low (down to $\left.0{ }^{\circ} \mathrm{C}\right)$ to high $\left(100{ }^{\circ} \mathrm{C}\right)$ temperature range. In contrast to biomaterials or waste materials Zhou et al. used disposable cashmere, a free carbon precursor for the synthesis of mesoporous $\mathrm{N}$ and $\mathrm{O}$ rich hierarchical electrode materials for high performance SCs. ${ }^{82}$ The utilization of high performance CNPs as an electrode material in miniaturized interdigitated energy storage devices is still at the infant stage and needs to be explored.

\subsection{Metal oxides/nitrides/hydroxides/sulphides based interdigitated electrodes}

Over the past two decades transition metal oxides, carbides, nitrides, sulphides and hydroxides have received significant attention as active electrode materials in energy storage devices due to their structural, electronic and mechanical properties, and multiple oxidation states. ${ }^{\mathbf{1 2 6 , 1 2 7}} \mathrm{MnO}_{2},{ }^{41} \mathrm{NiO}, \mathrm{Fe}_{3} \mathrm{O}_{4}, \mathrm{CoO}$, $\mathrm{V}_{2} \mathrm{O}_{5},{ }^{128} \mathrm{Al}_{2} \mathrm{O}_{3}, \mathrm{ZnO}, \mathrm{SnO}_{2},{ }^{129}$ and $\mathrm{MoO}_{2}{ }^{130}$ are the most widely 
a

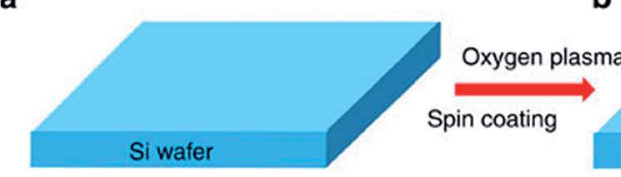

$f$

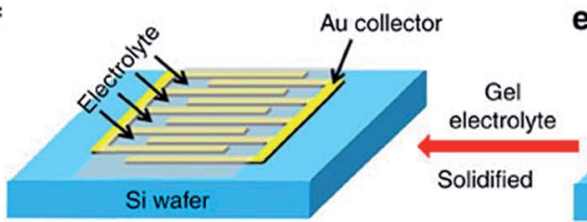

e

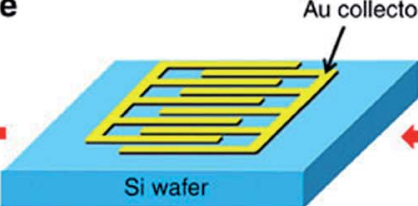

. b

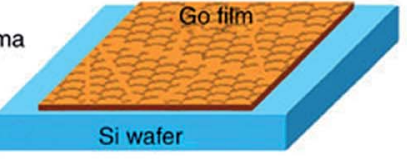

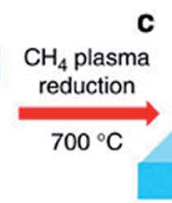

c

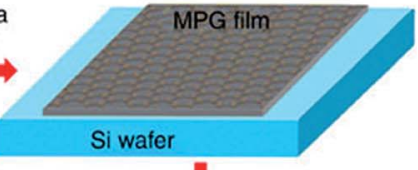

(1)

Masking and d
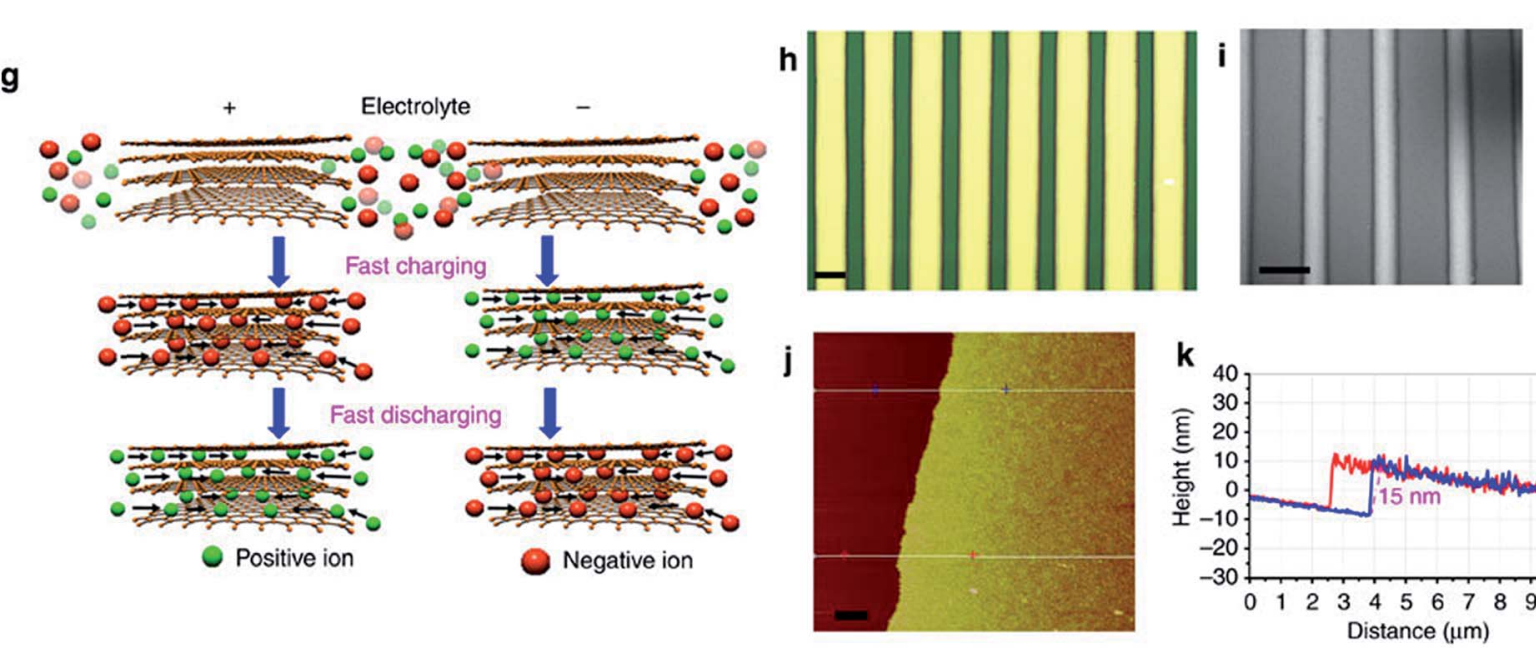

Si wafer
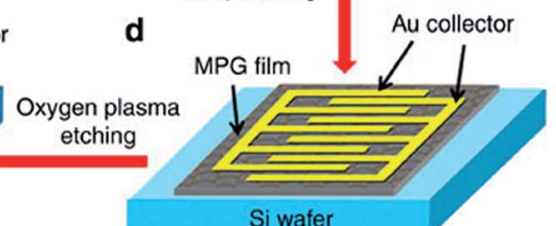

k

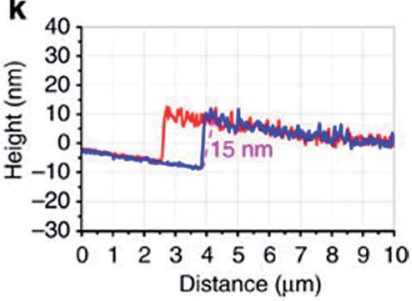

Fig. 13 Design of MPG-MSCs on silicon wafer. (a)-(f) Schematic illustration of the fabrication of MPG-MSCs made up of 30 interdigital fingers integrated onto a silicon wafer. The fabrication process flow includes (a) oxygen plasma surface treatment of silicon, spin-coating of the GO solution on surface-modified silicon, (b) reduction with $\mathrm{CH}_{4}$ plasma, (c) masking pattern and deposition of a gold current collector, (d) oxidative etching in oxygen plasma, (e) drop casting of a gel electrolyte (PVA/ $\mathrm{H}_{2} \mathrm{SO}_{4}$ ), (f) solidification of the gel electrolyte, (g) in-plane geometry of MPGMSCs, revealing that the ions between the electrode gaps can be rapidly transported along the planar graphene sheets with a short diffusion length, (h) optical and (i) SEM images of the microelectrode patterns. Scale bars, $200 \mathrm{~mm}$. (j) Atomic force microscopy image of the MPG film electrode after etching by oxygen plasma and removal of Au by a $\mathrm{KI} / \mathrm{I}_{2}$ aqueous solution. Scale bar, $1 \mathrm{~mm}$. (k) Uniform thickness of $\sim 15 \mathrm{~nm}$, indicated by the height profile of the MPG film. ${ }^{26}$ Adopted and modified from ref. 26 with permission from Macmillan Publishers Limited.

used transition metal oxides while the most studied noble metal oxides are $\mathrm{RuO}_{2}$ and $\mathrm{IrO}_{2} \cdot{ }^{\mathbf{1 2 6}}$ Metal nitrides are the least studied materials in energy storage devices in contrast to metal oxides due to their synthesis complexity and air-moisture sensitivity. Tong and coworkers reviewed the current trends in the synthesis prospects of transition metal nitrides. They summarized the recent progress of metal nitrides in energy storage applications along with future challenges. ${ }^{131}$ Recently, nanostructures of various metal nitrides, such as $\mathrm{VN},{ }^{132} \mathrm{TiN},{ }^{133,134}$ $\mathrm{Nb}_{4} \mathrm{~N}_{5}{ }^{135} \mathrm{FeN},{ }^{107} \mathrm{CrN},{ }^{136} \mathrm{Ni}_{3} \mathrm{~N},{ }^{137}$ and $\mathrm{Mo}_{2} \mathrm{~N},{ }^{138}$ have been explored as anode materials. ${ }^{127}$ Since the physicochemical properties of nanostructural materials significantly differ from those of bulk materials, ${ }^{128}$ particle size and morphology offer great opportunities to enhance capacitance performances. ${ }^{139}$ Various efforts have been made towards the synthesis of nanoparticles with controlled size and ordered morphology. ${ }^{36,140}$

Since its first application as an electrode material, $\mathrm{MnO}_{2}$ has shown promising and significant applications in energy storage devices due to its high theoretical capacitance of $1370 \mathrm{~F} \mathrm{~g}^{-1},{ }^{24}$ non-toxic nature and low cost as well. ${ }^{41,141}$ Jiang et al. used the composite of $\mathrm{MnO}_{2}$ and MWCNTs for the fabrication of 3D microelectrodes for application in on-chip MSCs. ${ }^{142} 3 \mathrm{D}$ microelectrodes were fabricated on silicon wafers by the combination of spin-coating, photolithography, pyrolysis, and electrochemical deposition techniques. Specific capacitance up to 238 $\mathrm{mF} \mathrm{cm} \mathrm{cm}^{-2}$ was reported with $81.8 \%$ capacitance retention after 6000 cycles. Wen et al. also fabricated a $\mathrm{MnO}_{2}$ based 3D microelectrode by the anodic electrodeposition technique to increase the electrode surface area, ${ }^{\mathbf{1 4 1}}$ since the performance of microscale energy storage devices is directly related to the electrode surface area.

The high cost and adverse environmental impacts of $\mathrm{RuO}_{2}$ hinders its commercial application even though it exhibits excellent specific capacitance with good electrochemical reversibility. ${ }^{\mathbf{1 2 6}}$ Since miniaturized devices require only minute amounts of $\mathrm{RuO}_{2}$, it shows great promise for use as an electrode material in MSCs. Dinh et al. reported the low resolution 
(distance between interdigitated fingers) fabrication of MSCs via a simple route based on hydrated $\mathrm{RuO}_{2}$ and MWCNT electrode material. ${ }^{\mathbf{1 4 3}}$ They used the widely adapted lift-up technique to pattern high resolution (10 $\mu \mathrm{m}$ interspace) interdigitated electrodes ${ }^{\mathbf{1 3 1}}$ on traditionally fabricated low resolution electrode patterns for MSCs as demonstrated in Fig. 14. Low resolution MSCs were fabricated by using an insulating SU8 masking layer via the photolithography technique followed by the lift-up technique. The MSCs exhibited a discharge rate as high as $10000 \mathrm{~V} \mathrm{~s}^{-1}$ with a high specific capacitance of $3 \mathrm{mF} \mathrm{cm} \mathrm{cm}^{-2}$. Warren et al. adopted the atomic layer deposition technique for the coating of $\mathrm{RuO}_{2}$ on vertically aligned CNTs and the hybrid material was further utilized in SCs. ${ }^{144}$ Wang et al. fabricated a 3D MSC based on tubular hydrous $\mathrm{RuO}_{2}$ by utilizing an ICP etching technique on silicon wafers. MSCs were further on-chip integrated and exhibited a specific capacitance of $23 \mathrm{mF} \mathrm{cm}{ }^{-2}$ at $10 \mathrm{~mA} \mathrm{~cm}^{-2} .^{145}$

Silicon nanostructures, such as silicon nanowires (SNWs), ${ }^{\mathbf{1 4 6}}$ hyper-branched silicon nanowires, silicon nano-trees and silicon nanotubes sparked tremendous attention for technological innovations in miniaturization of energy storage devices due to their high surface area, chemical and thermal stability, corrosion resistance, high power pulse, and biocompatible and biodegradable nature. ${ }^{\mathbf{1 4 7}}$ One such synthesis of porous silicon nanowires via the well-known low temperature lithographic technique was described by Alper et al. ${ }^{148}$ These SNWs were further applied as an electrode material on ultrathin carbon sheath and demonstrated a specific capacitance of $325 \mathrm{mF} \mathrm{cm}^{-2}$ at a current density of $1 \mathrm{~mA} \mathrm{~cm}{ }^{-2}$.
Apart from controlling the particle size and morphology, an appropriate electrolyte is also a key component for increasing the energy efficiency of MSCs. The electrochemical stability of electrolytes limits the ionic performance of MSCs. Aradilla et al. used a protic ionic liquid (triethylammonium bis(trifluoromethylsulfonyl)imide; $\mathrm{NEt}_{3} \mathrm{H}$ TFSI) as an electrolyte for planar MSCs while using chemical vapor grown silicon nanowires as the electrode material. ${ }^{\mathbf{1 4 7}}$ The resulting MSCs demonstrated a high power density of $4 \mathrm{~mW} \mathrm{~cm}^{-2}$ for a wide cell voltage range of $4 \mathrm{~V}$ and long cycle life (several million GCD cycles). In another report Aradilla et al. employed an alternative aprotic ionic liquid ( $N$-methyl- $N$-propylpyrrolidinium bis(trifluoromethylsulfonyl)imide) ( $\mathrm{PYR}_{13} \mathrm{TFSI}$ ) as an electrolyte for silicon nanowires and silicon carbide nanowires based MSCs. ${ }^{\mathbf{1 4 6}}$ The enhanced compatibility between the electrode and electrolyte improved the stability of MSCs and $75 \%$ of the specific capacitance was retained after $8 \times 10^{6}$ GCD cycles. The MSCs were reported to show good capacitive behavior and exhibited a power density of $182 \mathrm{~mW} \mathrm{~cm} \mathrm{~cm}^{-2}$ and specific energy of $0.19 \mu \mathrm{J}$ $\mathrm{cm}^{-2}$ at an operating potential window of $4 \mathrm{~V}$.

Chatterjee et al. reported the corrosion resistance and electrochemical stability of a silicon nanomaterial and graphene hybrid. ${ }^{\mathbf{1 4 9}}$ This hybrid material was further applied to the fabrication of high power on-chip SCs as an electrode material. It exhibited power density of $65 \mathrm{~kW} \mathrm{~kg}^{-1}$ and energy density of $10 \mathrm{~W} \mathrm{~h} \mathrm{~kg}{ }^{-1}$, comparable with other carbon nanomaterials based SCs. The silicon nanomaterial assisted the fabrication of few layer graphene at high temperatures $\left(>850{ }^{\circ} \mathrm{C}\right)$ in acetylene and resulted in the enhancement of corrosion resistance against aqueous, organic and ionic environments.

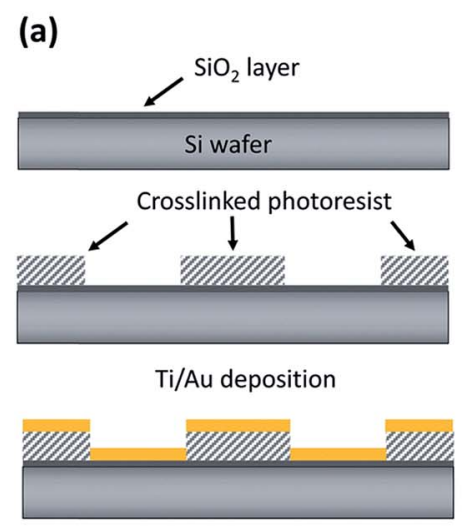

(b)

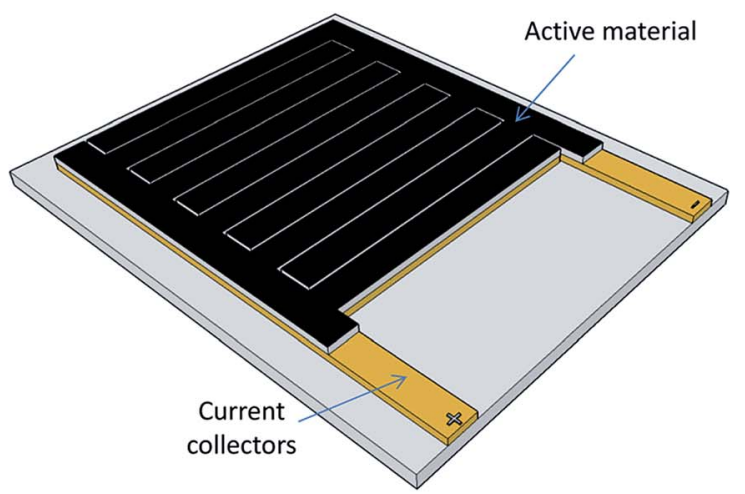

(c)

(d)
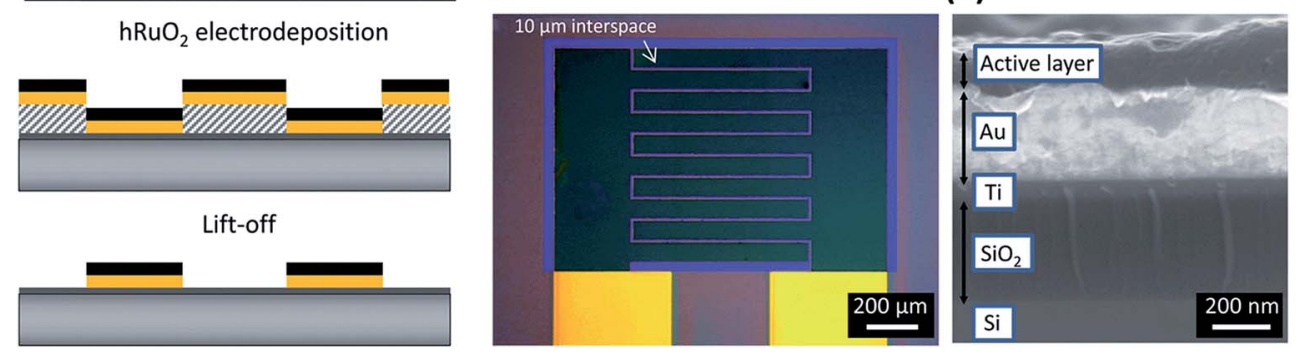

Fig. 14 Fabrication and design of MSCs. (a) Simplified illustration of the fabrication process using the lift-off process of the active material for a high-resolution pattern (not to scale); schematic (b) and optical image (c) of the micro-device, (d) scanning electron microscopy image of the cross-section of the electrode. ${ }^{143}$ Adopted from ref. 143 with permission from The Royal Society of Chemistry. 
Fabrication of asymmetric electrodes in MSCs has emerged as a promising approach to increase the energy density without affecting the power density and cycle life at a wider operating voltage window, ${ }^{61,115,150}$ since different electrodes offer different potential windows which enhance the operational voltage and ultimately energy density. Tong and coworkers used vanadium nitride (VN) nanowires as a high energy anode material in contrast to carbon materials and $\mathrm{VO}_{x}$ nanowires as a cathode material for the fabrication of high energy density ASCs in a LiCl/PVA gel electrolyte. ${ }^{132}$ They used VN due to its negative potential working window, higher specific capacitance $(1340 \mathrm{~F}$ $\left.\mathrm{g}^{-1}\right)$ than CNPs and also higher electrical conductivity $\left(\sigma_{\text {bulk }}=\right.$ $1.67 \times 106 \Omega^{-1} \mathrm{~m}^{-1}$ ) than commonly used metal oxides. Porous VN nanowires were fabricated in two steps via hydrothermal fabrication of $\mathrm{VO}_{x}$ nanowires on a carbon cloth. Then $\mathrm{VO}_{x}$ nanowires were reduced to $\mathrm{VN}$ nanowires by post annealing in ammonia at $600{ }^{\circ} \mathrm{C}$ for $1 \mathrm{~h}$. More significantly the authors overcame the negative aspect of VN (aqueous instability due to oxidation) by using the LiCl/PVA gel electrolyte. The water content and structure pulverization were minimized by the direct contact of electrode materials and substrate during operational cycling. They reported that even after 10000 cycles only $12.5 \%$ capacitance was decreased. The ASCs exhibited a high energy density of $0.61 \mathrm{~mW} \mathrm{~h} \mathrm{~cm} \mathrm{~cm}^{-3}$ at $0.5 \mathrm{~mA} \mathrm{~cm}^{-2}$ current density and $0.85 \mathrm{~W} \mathrm{~cm}^{-3}$ power density at $5 \mathrm{~mA} \mathrm{~cm} \mathrm{~cm}^{-2}$ current density.

In another report, Tong and coworkers introduced oxygen vacancies in hematite $\left(\alpha-\mathrm{Fe}_{2} \mathrm{O}_{3}\right)$ nanorods in order to improve the electrochemical performance in ASCs as an anode material. ${ }^{151} 1 \mathrm{D}$ nanorods were directly grown on a carbon cloth in two steps. First, a hydrothermal method was used to fabricate vertically aligned akaganeite $(\mathrm{FeOOH})$ nanorods on a conductive substrate (carbon cloth). Thermal treatment at $400{ }^{\circ} \mathrm{C}$ in a $\mathrm{N}_{2}$ atmosphere resulted in oxygen deficient $\mathrm{Fe}_{2} \mathrm{O}_{3}$ nanorods. $\mathrm{Fe}_{2} \mathrm{O}_{3}$ nanorods were used as the anode material while $\mathrm{MnO}_{2}$ electrodeposited on carbon cloth was used as the cathode material in LiCl/PVA gel electrolyte. 95\% of initial capacitance $\left(382.7 \mathrm{mF} \mathrm{cm}^{-2}\right.$ ) was retained after 10000 cycles. The ASCs based on $\mathrm{MnO}_{2} / / \mathrm{Fe}_{2} \mathrm{O}_{3}$ demonstrated a maximum energy density of $0.41 \mathrm{~mW} \mathrm{~h} \mathrm{~cm} \mathrm{~m}^{-3}$ at $0.5 \mathrm{~mA} \mathrm{~cm}^{-2}$ current density and a maximum power density of $0.1 \mathrm{~W} \mathrm{~cm}^{-3}$ at $6 \mathrm{~mA} \mathrm{~cm} \mathrm{~cm}^{-2}$ current density.

\subsection{Conducting polymer based interdigitated electrodes}

Conducting polymers, such as PANI, PPy, polyacetylene, poly(3,4-ethylenedioxythiophene) (PEDOT), polythiophene, and their derivatives are widely used as promising electrode materials in energy storage devices due to the $\pi$ conjugated structures of such polymers. ${ }^{152}$ The availability of several redox states in conducting polymers offers tailorable electrical conductivity from insulator to metal and holds prospective applications in pseudocapacitors. ${ }^{153}$ Tremendous efforts have been devoted to enhance the specific capacitance and stability of MSCs based on conductive polymer electrode materials. Aradilla et al. fabricated a symmetric MSC based on a PEDOT coated SNW electrode material and an ionic liquid $N$-methyl- $N$ - propylpyrrolidinium bis(trifluoromethylsulfonyl)imide as an electrolyte. ${ }^{154}$ Improvement in the performance and stability ( $80 \%$ after $>1000$ cycles) of the MSCs in contrast to other SNWs based SCs was observed. The MSCs exhibited a specific energy of $10 \mathrm{~W} \mathrm{~h} \mathrm{~kg} \mathrm{~W}^{-1}$ at a voltage window of $1.5 \mathrm{~V}$ with a maximal power density of $85 \mathrm{~kW} \mathrm{~kg}{ }^{-1}$. PEDOT is one of the widely explored conducting polymers for the fabrication of flexible MSCs since it offers a wide potential operating window for electrochemical activity in different electrolytes (aqueous, organic and ionic liquids). ${ }^{155}$ Nevertheless, MSCs composed of PEDOT based electrodes suffer from low specific capacitance in contrast to other conducting polymers owing to their high molar mass and low doping level. ${ }^{155}$ Recently Kurra et al. fabricated PEDOT based MSCs with excellent frequency characteristics of $400 \mathrm{~Hz}$ and energy density as high as $7.7 \mathrm{~mW} \mathrm{~h}$ $\mathrm{cm}^{-3} \cdot{ }^{71}$ MSCs were fabricated by using the combination of conventional photolithography and the electrochemical deposition technique. MSCs were fabricated on a plastic polyethylene naphthalate (PEN) sheet surface and electrochemical deposition was carried out before the lift-off process. The electrode architecture was optimized by using a surfactant-mediated process with sodium dodecyl sulfate (SDS) as the surfactant. More than $80 \%$ flexibility and stability of the PEDOT MSCs was found to be retained after 10000 cycles with a coulombic efficiency of $100 \%$.

Fabrication of 3D hierarchically nanostructured hydrogels of conductive polymers is of growing interest due to the formation of conducting frameworks which provide an ideal interface between the phases of electronic and ionic transport pathways, ${ }^{\mathbf{1 5 6}}$ especially in biological systems. Hence, these can be successfully applied in biomedical devices. ${ }^{156}$ However, such hydrogels generally show poor mechanical properties which hinder their practical applications in flexible energy storage devices. Shi et al. fabricated the 3D nanoarchitectures of PPy as hydrogels by using an interfacial polymerization technique in a solution-based approach and utilized these as an electrode material in flexible SCs. ${ }^{157}$ The as-fabricated PPy hydrogel provided tunable electrochemical and mechanical properties as well as scalable processability by controlling the synthesis parameters.

Most of the research on the fabrication of MSCs mainly concentrate on the engineering of electrode materials and their architecture. However, practical integration and utilization in miniature energy storage devices still remains difficult due to the comparatively large distribution of the electrical routing path and contact pads in energy storage devices. ${ }^{25}$ Only few reports are available for the compact encapsulation of the electrolyte at the micro-scale. In addition, encapsulation of the electrolyte material in micro-scale energy storage devices is also an issue. Compact integration of MSCs at the micro-level $(\approx 720$ $\mu \mathrm{m} \times 720 \mu \mathrm{m} \times 50 \mu \mathrm{m})$, i.e. miniaturization of SCs and integration of MSCs, was achieved by Meng et al. ${ }^{31}$ They overcame the issues of micro-scale electrolyte encapsulation in two steps. Firstly a large area of the device was coated with a solid state electrolyte and then MSCs were diced out. The "top down" micro-fabrication was carried out on the top side of a liquid crystal polymer as a current collector and on the bottom side as 
an electrical contact pad and both sides were connected via a hole contact. The overall process is shown in Fig. 15. PANI nanowires were used as electrode materials. Multiple MSCs were obtained by a single fabrication technique. Electrode thickness showed a linear impact on the energy storage performance of the microdevice.

\subsection{Hybrid materials based interdigitated electrodes}

Metal oxides and conducting polymers offer higher specific capacitance than carbon materials due to their pseudocapacitive nature. Economic MSCs based on carbon nanostructures generally have a major drawback of low capacitance and energy density. ${ }^{3}$ Graphene is widely used a carbon based nanomaterial in energy storage devices. However, its tendency to easily aggregate hinders the superb performance of the devices for superior applications in energy storage. ${ }^{158,159}$ The practical application of conducting polymers or metal oxides as electrode materials in MSCs also suffers from few limitations. Conducting polymers exhibit some drawbacks such as poor substrate binding, less cyclic stability in solution and high internal resistance, while the poor conductivity of metal oxides inhibits their applications in advanced technological devices. A significant issue for the miniaturization of energy storage devices is the need to improve the specific capacitance and energy density within the footprint area of the device while maintaining cyclic stability. ${ }^{43}$ A general approach reported by various research groups to address these short-comings is to increase the conductivity of pseudocapacitive materials by incorporating highly conductive materials either by composite formation or direct doping. ${ }^{34,39,83,107,144,160,161}$ The introduction of conductive materials provides an opportunity for superior conductivity and also enhances the electrochemical performances of devices. ${ }^{\mathbf{1 6 2}}$ Carbon nanostructures such as CNTs and graphene have been largely explored for the fabrication of composite materials.

\section{a}
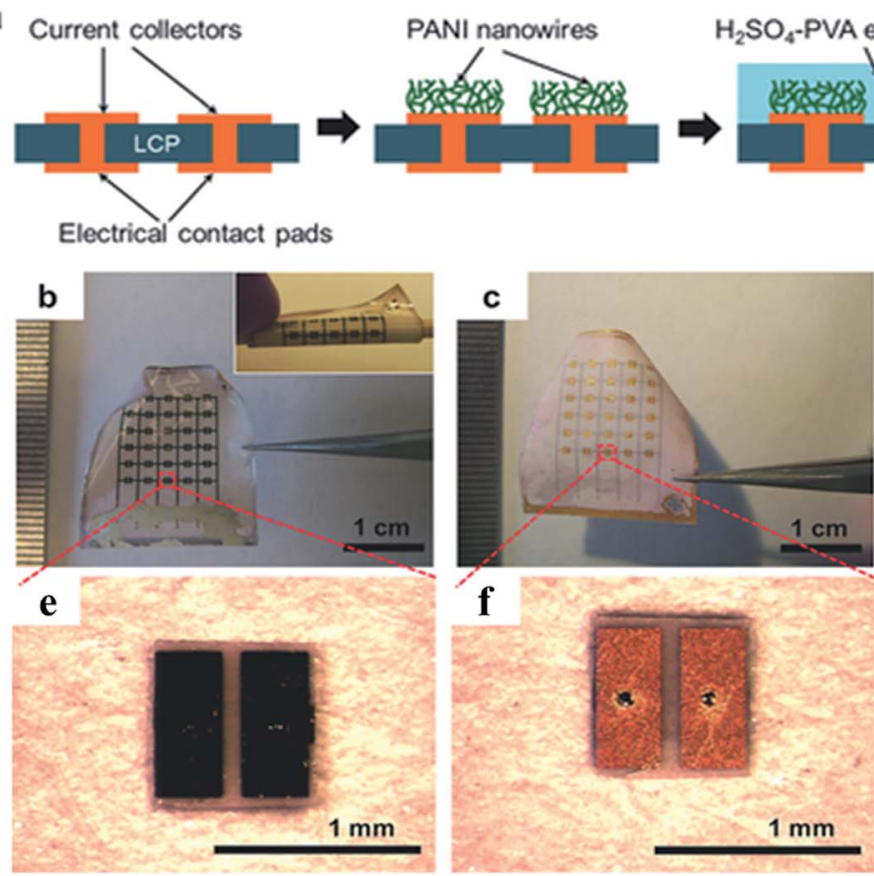

d
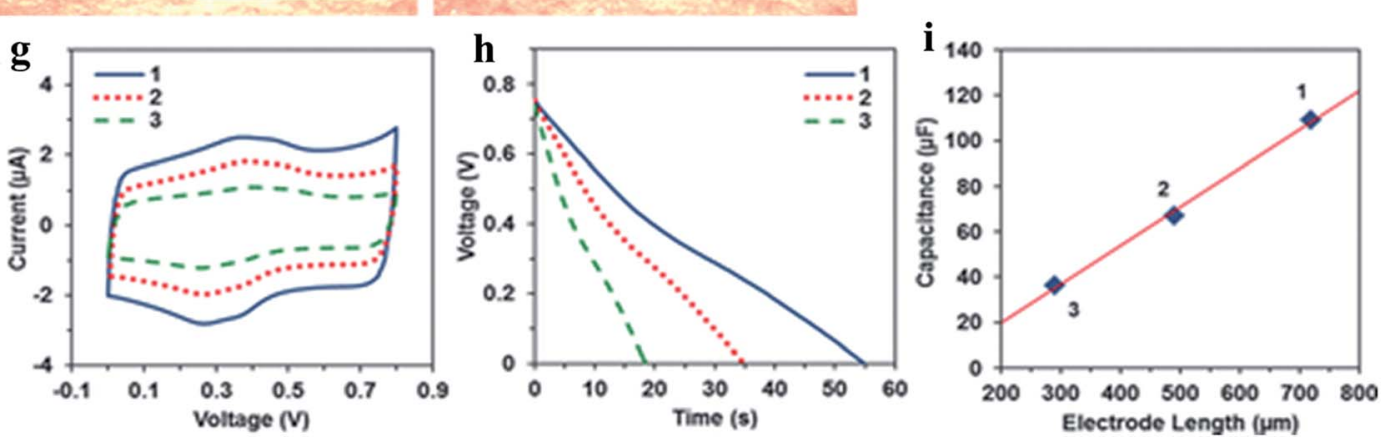

Fig. 15 (a) Fabrication of integrated 3D MSCs with through via bottom electrode contact (cross-sectional view). Photographs of (b) the topside and (c) backside of an LCP sheet with multiple embedded MSCs. The inset of (b) shows the mechanical flexibility of the composite sheet. Optical microscope images of (e) the topside and (f) backside of a single device when cut out. (d) Photographs of the various-sized devices $(\approx 720 \mu \mathrm{m} \times$ $720 \mu \mathrm{m}, 720 \mu \mathrm{m} \times 490 \mu \mathrm{m}$, and $720 \mu \mathrm{m} \times 290 \mu \mathrm{m}$ ) obtained by sequentially cutting down a single device. (g) CV curves at $20 \mathrm{mV} \mathrm{s}^{-1}$ and (h) galvanostatic discharge curves at $\approx 1.5 \mu \mathrm{A}$ for different device sizes. (i) Relationship between the device capacitance and electrode length. ${ }^{31}$ Adopted and modified from ref. 31 with permission from WILEY-VCH Verlag GmbH \& Co. KGaA, Weinheim. 
Significant advancements have been documented for on-chip integrated solid state and flexible microelectrodes. ${ }^{163}$

Wang et al. recently addressed the above-mentioned issue by combining a high surface area Si MEMS as a current collector with a close-grained $\mathrm{RuO}_{2} / \mathrm{CNT}$ composite as an electrode material to fabricate on-chip 3D high energy density MSCs. ${ }^{\mathbf{1 6 1}}$ The ICP (inductively coupled plasma) technique was used to fabricate 3D MSCs and the current collector was fabricated by using the FIB (focused ion beam) technique. Ripple-like composite film of $\mathrm{RuO}_{2} / \mathrm{CNTs}$ with a high specific surface area and conductivity was grown on a "grassy prominence" $\mathrm{Si}$ microstructure template via in situ electrodeposition. The footprint of the $3 \mathrm{D}$ array structure was $2 \times 2 \mathrm{~mm}$ that contained 832 vertically aligned microelectrode pillars. The "grassy prominence" of the substrate helped to bind and deposit $\mathrm{RuO}_{2}$ nanoparticles on the CNT surface. The specific capacitance and specific power density of these MSCs were reported to be 37.23 $\mathrm{mF} \mathrm{cm} \mathrm{cm}^{-2}$ and $19.04 \mathrm{~mW} \mathrm{~cm}^{-2}$, respectively, at a relatively large discharge rate of $50 \mathrm{~mA} \mathrm{~cm}{ }^{-2}$. Chen et al. fabricated MSCs based on a new electrode material $\mathrm{MnO}_{2} / \mathrm{b}-\mathrm{PEDOT} / \mathrm{MWCNT}$ nano-composites. ${ }^{\mathbf{1 6 4}}$ The MWCNT surface was decorated with hollow b-PEDOT spheres and then wrapped by $\mathrm{MnO}_{2}$ nanograins that resulted in a network-like structure. These nanocomposites were further applied as an electrode material for the fabrication of flexible MSCs which demonstrated a good specific capacitance of $110 \mathrm{~F} \mathrm{~g}^{-1}$ at a current density of $2 \mathrm{~A} \mathrm{~g}^{-1}$. Fiorentino et al. fabricated highly reproducible, 3D solid state MSCs based on the hybrid composite of $\mathrm{Al}_{2} \mathrm{O}_{3}$ /MWCNT and TiN as a conformal counter-electrode. ${ }^{42}$

The poor electrical conductivity of $\mathrm{MnO}_{2}$ obstructs its applications in advanced technologies. To improve the electrical conductivity $\mathrm{MnO}_{2}$ is usually combined with CNPs. Zeng et al. synthesized a $\mathrm{MnO}_{2}$ nanowires and nano-porous gold hybrid electrode material and further applied it to the fabrication of solid-state interdigitated MSCs which resulted in a relatively high power density of $3.4 \mathrm{~W} \mathrm{~cm}^{-3} \cdot{ }^{\mathbf{1 6 5}}$ Furthermore Nan et al. used a hierarchical composite of a binary metal oxide spinel, $\mathrm{NiMn}_{2} \mathrm{O}_{4}$ nanosheets, and CNTs for the fabrication of high performance AMSCs. They achieved a high energy density of $60.69 \mathrm{~W} \mathrm{~h} \mathrm{~kg}^{-1}$ and power density of $874 \mathrm{~W} \mathrm{~kg}^{-1}$ at $1.7 \mathrm{~V}$ voltage window. ${ }^{160}$ Application of $\mathrm{MnO}_{2}$ and MWCNT composites in 3D microelectronics was also reported by Jiang et al. for on-chip MSC applications. ${ }^{142}$ For $\mathrm{MnO}_{2}$ and conductive polymer based composite materials, the high aspect ratio of the nanoparticles leads to collapse of nanoparticles and hence the active surface area gets reduced which on the whole resulted in the limited accessibility of the electrolyte. ${ }^{\mathbf{1 6 6}}$ A sandwich-like Ag/ PEDOT:PSS/ $\mathrm{MnO}_{2}$ electrode material was fabricated via the LBL assembly by Thomas and co-workers to achieve a large surface area. ${ }^{134,167}$ Introduction of poly(styrenesulfonate) (PSS) enhances the solubility of PEDOT and hence spin-coating becomes easy. $\mathrm{Ag}$ nanowires were introduced to increase the conductivity of the composite material. The overall assembly enhances the facile transport of electrons via a network structure. These SCs demonstrated a specific capacitance of $862 \mathrm{~F}$ $\mathrm{g}^{-1}$ at $2.5 \mathrm{~A} \mathrm{~g}^{-1}$ current density. ${ }^{165}$
Dinh et al. demonstrated a route towards the integration of all-solid-state MSCs exhibiting ultrahigh-energy-density by using a hybrid material of hierarchical carbon nanowalls and hydrous $\mathrm{RuO}_{2} \cdot{ }^{168}$ They decorated porous $\mathrm{RuO}_{2}$ on the outer surface of vertically aligned carbon nanowalls. Under optimized experimental conditions, outstanding specific capacitance of $1097 \mathrm{mF} \mathrm{cm}^{-2}$ was achieved and the energy density achieved was comparable with that of lithium ion batteries. Hence, this could be a step towards the bridging between batteries and SCs.

The selection of an optimum electrode material is a crucial step to achieve high energy density. Hybrid materials were widely employed as electrode materials for MSCs but they also suffer from some limits such as difficulty in further reducing the ionic diffusion pathway and on-chip integration with high energy density. One such approach to optimize hybrid material performance was proposed by Kim et al. ${ }^{\mathbf{1 6 9}}$ They fabricated a flexible interdigitated MSC over a PET substrate using a MWCNT $/ \mathrm{V}_{2} \mathrm{O}_{5}$ nanowire composite with $10 \mathrm{vol} \% \mathrm{~V}_{2} \mathrm{O}_{5}$ nanowires as the electrode material in PVA-LiCl solid electrolyte and integrated it with a $\mathrm{SnO}_{2}$ nanowire based UV sensor. The volumetric capacitance was found to be $80 \mathrm{~F} \mathrm{~cm}^{-3}$ at a scan rate of $10 \mathrm{mV} \mathrm{s}^{-1}$ and $0-0.8 \mathrm{~V}$ potential window, along with $82 \%$ retention of initial capacitance after 10000 cycles at a current density of $11.6 \mathrm{~A} \mathrm{~cm}^{-3}$. Such an MSC showed a high volumetric

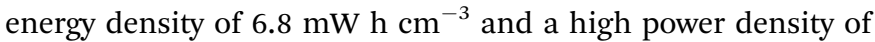
$80.8 \mathrm{~W} \mathrm{~cm}^{-3}$ at a current density of $1.8 \mathrm{~mA} \mathrm{~cm}^{-3}$. The MSC also showed good electrochemical stability against both convex and concave bending deformation at a radius of $1.5 \mathrm{~mm}$ and retained $94 \%$ of its original capacitance after 1000 bending cycles at a bending radius of $7 \mathrm{~mm}$. The authors also demonstrated operation of a UV sensor for $130 \mathrm{~s}$ using this MSC. ${ }^{169}$

Another major challenge in real life applications of MSCs is their lower energy density in comparison to microbatteries. ${ }^{\mathbf{1 7 0}} \mathrm{In}$ this regard the redox chemistry of CNPs having a higher accessible surface area has been successfully applied to enhance the volumetric capacitance of CNPs based electrode materials. ${ }^{25,171}$ Much effort has been taken to enhance the cycle life and rate capabilities of CNPs based MSCs. However their low volumetric energy density is still a key issue to be solved. Fabrication of porous and highly conductive electrode materials along with high packing density can enhance volumetric energy density in MSCs. Chen and co-workers reported the enhancement of volumetric energy density as high as $\sim 6.3 \mathrm{~mW} \mathrm{~h} \mathrm{~cm} \mathrm{~cm}^{-3}$ in a solid-state MSC based on hierarchically structured carbon microfibers. ${ }^{83}$ They further integrated it into miniaturized flexible energy storage devices. Microfibers with an interconnected network structure were fabricated hydrothermally with carboxylated SWCNTs, GO and ethylene diamine. The composite microfibers exhibited high packing density, electrical conductivity and higher exposed surface area due to the novel interconnected structure as demonstrated in Fig. 16(b). The specific volumetric capacity of the microfibers was found to be $305 \mathrm{~F}$ $\mathrm{cm}^{-3}$ in $\mathrm{H}_{2} \mathrm{SO}_{4}$ and $300 \mathrm{~F} \mathrm{~cm}^{-3}$ in $\mathrm{PVA}^{-} \mathrm{H}_{3} \mathrm{PO}_{4}$. Flexible MSCs were fabricated as demonstrated in Fig. 16. More notably the power density was maintained with a long cycle life. This solid state MSC demonstrated excellent electrical conductivity and 
high packing density while maintaining a large ion-accessible surface.

Several approaches have been explored for the sufficient utilization of the high capacitance of conducting polymers and also to overcome the drawbacks of their poor cyclic stability. Fabrication of either micro/nanostructures with diverse morphology or metal oxides and CNPs based composite materials has been reported. ${ }^{\mathbf{1 5 2}}$ However, the nanostructures of conducting polymers are less stable and exhibit poor conductivity. ${ }^{172}$ CNPs have been largely applied as conductive scaffolds in conductive polymer based electrode materials. For instance, $\mathrm{Yu}$ et al. fabricated a hybrid architecture of PANI-rGO with controlled morphology on a N-doped carbon fabric. ${ }^{173}$ Nitrogen doping in carbon fabric was achieved by 2 min exposure in a nitrogen/oxygen atmosphere by pulsed power supply (160 W). PANI nanorods were grown on N-doped carbon fabric by in situ polymerization of aniline, then dipped in GO suspension and finally reduced by $\mathrm{HI}$ to obtain the hybrid nanostructure of PANI-rGO as demonstrated in Fig. 17. The hybrid nanorod array was applied as an electrode material in $\mathrm{H}_{2} \mathrm{SO}_{4}$ electrolyte to fabricate flexible MSCs. The authors demonstrated that coating of rGO provided resistance against the mechanical deformation and volume changes for the nanowire array of PANI during the long-term charge/discharge process. In addition the strong $\pi-\pi$ interaction between PANI and the rGO basal plane provided a good electrical pathway. The MSCs exhibited enhanced capacitance with a specific capacitance of $1145 \mathrm{~F} \mathrm{~g}^{-1}$, energy density of $25.4 \mathrm{~W} \mathrm{~h} \mathrm{~kg}^{-1}$ and a maximum power density of 92.2 $\mathrm{kW} \mathrm{kg}^{-1} \cdot 94 \%$ of the initial capacitance for such MSCs was found to be retained after 5000 cycles.

Polypyrrole holds great potential for use as an electrode material in hybrid composites due to its good stability, high conductivity, ease of fabrication and low cost as well. Various PPy-CNP composites have been explored as potential electrode materials in energy storage devices with enhanced electrochemical performances. ${ }^{174}$ Raj et al. demonstrated a simple approach for the fabrication of flexible MSCs by using commercially available polymer lapping film (aluminum oxide bonded polyester) of 4000 grit (particle size $3 \mu \mathrm{m}$ ) as a flexible substrate. ${ }^{174}$ A graphite flakes-PPy composite was used as an electrode material in PVA- $\mathrm{H}_{3} \mathrm{PO}_{4}$ gel electrolyte. The graphite flakes-PPy hybrid composite was fabricated in two simple steps. Firstly, a graphite rod was soothingly scraped on the surface of lapping film and then after removing loosely bound graphite flakes $p$-toluene sulfonate ( $p$ TS)-doped PPy was electrochemically deposited on it. Electrical connections were made by the application of conductive silver paste at appropriate places. The as-fabricated electrode exhibited a capacitance of $37 \mathrm{mF} \mathrm{cm} \mathrm{cm}^{-2}$ in $1 \mathrm{M} \mathrm{H}_{2} \mathrm{SO}_{4}$ electrolyte (half cell configuration) and $23 \mathrm{mF}$ $\mathrm{cm}^{-2}$ in full cell configuration. A planar cell configuration with (a)

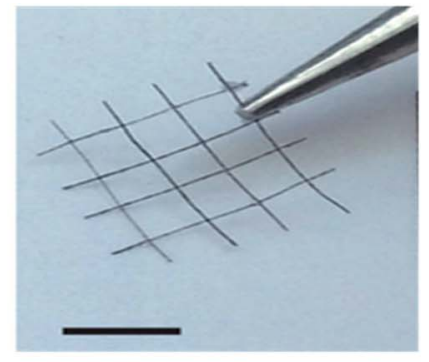

(b)

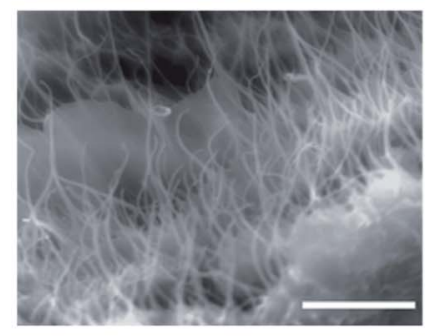

(c)

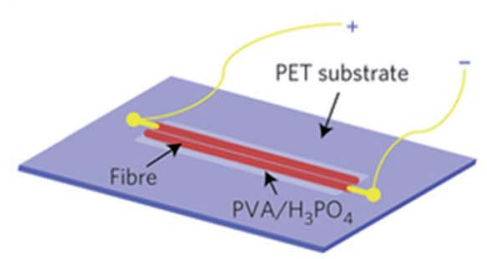

(d)

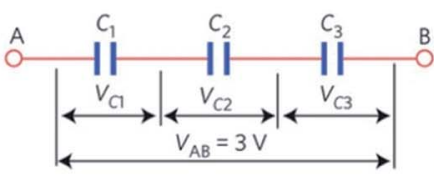

(e)
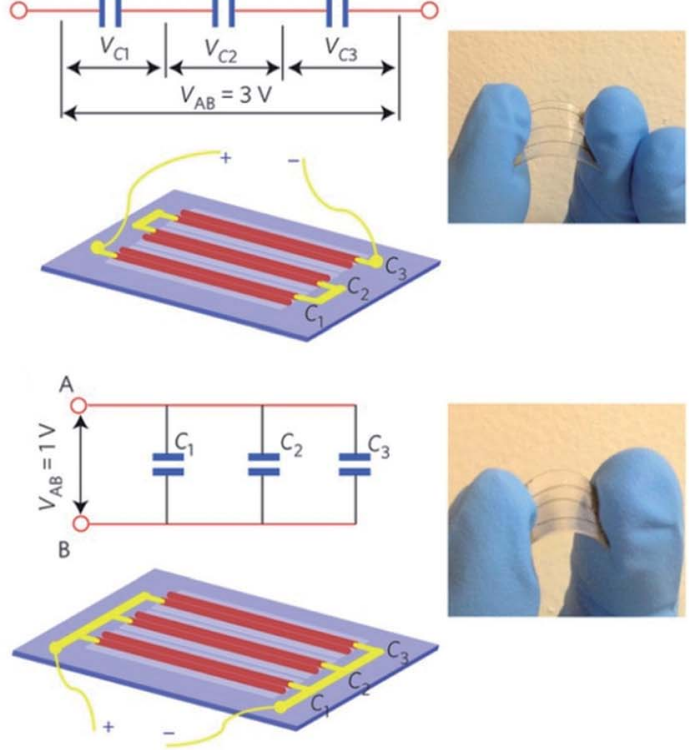

Fig. 16 (a) Knitted textile fabricated from fibers (scale bar $0.5 \mathrm{~cm}$ ); (b) microstructures of the carbon hybrid microfibers showing the attachment of SWCNT bundles on the edges and surface of rGO; (c) schematic representation of a MSC constructed using two fiber 3-electrodes on a polyester (PET) substrate; (d) schematic, equivalent circuit and photograph of the three MSCs connected in series; (e) schematic, equivalent circuit and photograph for the scalable integration of multiple fibers in MSCs in parallel. ${ }^{83}$ Adopted and modified from ref. 83 with permission from Macmillan Publishers Limited. 


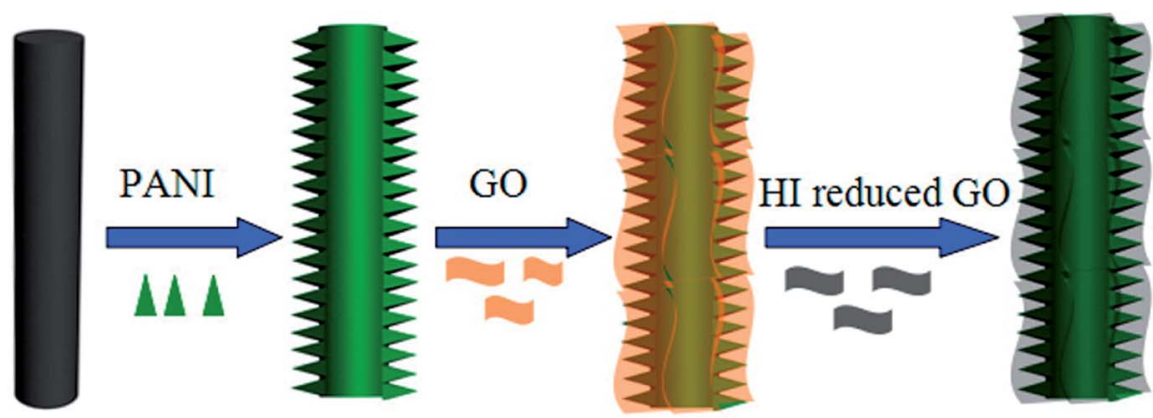

\section{N-doped eCFC PANI coated fibers GO coated PANI/eCFC RGO/PANI/eCFC}

Fig. 17 Schematic representation for the fabrication of an rGO/PANI hybrid nanoarray on N-doped carbon fabric. ${ }^{173}$ Adopted from ref. 173 with permission from the American Chemical Society.

a solid electrolyte (PVA- $\left.\mathrm{H}_{3} \mathrm{PO}_{4}\right)$ exhibited a maximum capacitance of $6 \mathrm{mF} \mathrm{cm} \mathrm{cm}^{-2}$. Aradilla et al. fabricated a PPy coated silicon nanotree (SiNTr) hybrid for energy storage application in MSCs. ${ }^{175}$ The SiNTr was fabricated by using the CVD method on silicon substrates, followed by coating with PPy via electropolymerization of pyrrole. An aprotic ionic liquid ( $N$-methyl- $N$ propylpyrrolidinium bis(trifluoromethylsulfonyl)imide; PYR13TFSI) was used as the electrolyte. The specific capacitance of such a fabricated MSC was $14 \mathrm{mF} \mathrm{cm}{ }^{-2}$ with $15 \mathrm{~mJ} \mathrm{~cm}^{-2}$ energy density at $1 \mathrm{~mA} \mathrm{~cm}{ }^{-2}$ current density.

Over the past few years sustainable and low cost conductive polymer based flexible electrode materials have been gaining immense attention in microscale energy storage devices. However, the low volumetric capacitance in contrast to CNPs and carbides-based electrode materials still constitutes a challenge. One such approach for the fabrication of freestanding and flexible electrodes was demonstrated by Strømme and coworkers. ${ }^{176}$ They tailored the surface functionalities of the most abundant biopolymer nanocellulose fibers (u-NCFs) with anionic carboxylate groups (a-NCFs) or cationic quaternary amine groups (c-NCFs). NCFs-PPy composites were fabricated by the polymerization of pyrrole in the presence of all the three types of NVFs (u-NCFs, c-NCFs, and a-NCFs). The composites exhibited porous structures with numerous intertwined fibers. They showed that surface modification of NCFs can significantly affect the mechanical properties of composites. Cationic modification leads to higher flexibility, tensile strength, density and a lower surface area while anionic modification resulted in lower flexibility and higher surface area. PPy-NCF composite papers $(\sim 1.0 \mathrm{~cm} \times 1.0 \mathrm{~cm})$ separated by ordinary filter paper were employed as electrodes for the fabrication of flexible SCs with Pt foil as the current collector. Up to $122 \mathrm{~F} \mathrm{~cm}^{-3}$ volumetric capacitance and $127 \mathrm{~F} \mathrm{~g}^{-1}$ full electrode-normalized gravimetric capacitance was achieved at $300 \mathrm{~mA} \mathrm{~cm}{ }^{-2}$ current density.

ASCs, a modified version of traditional SCs, demonstrated superior performance in terms of energy density since they can be operated at a higher voltage window in aqueous solution. ${ }^{177}$ Fiber based ASCs have scarcely been reported due to the complication in the fabrication of compatible fiber electrodes. Gao and co-workers were the first to fabricate graphene based flexible MSCs with high volume energy density $(11.9 \mathrm{~mW} \mathrm{~h}$ $\mathrm{cm}^{-3}$ ) and cyclic stability. ${ }^{115}$ Hybrid materials of $\mathrm{MnO}_{2}$-graphene and CNT graphene were used for the fabrication of asymmetric electrodes in a PVA/LiCl gel electrolyte by using facile wet-spinning assembly. Apart from graphene nanosheets or rGO, graphene quantum dots have also been used in energy storage applications due to the high density of edge defects and pronounced quantum confinement. Graphene quantum dots (anionic electrode material) and PANI nanofibers (cationic electrode material) based ASCs were fabricated by Liu et al. using the electro-deposition technique on an interdigital finger gold electrode. ${ }^{39}$

CNPs have been largely explored as anode materials in ASCs, since they offer high surface area with excellent electrical conductivity. Although high power density was achieved by using CNPs as electrode materials, the low energy density of ASCs is still a major concern due to the low capacitance of CNPs. ${ }^{30,148}$ Synthesis of high capacitance anode materials is a key issue for the fabrication of high energy ASCs. Hematite ( $\alpha$ $\mathrm{Fe}_{2} \mathrm{O}_{3}$ ) exhibits high theoretical specific capacitance with the advantage of green and economic nature but its poor electrical conductivity $\left(\mathrm{ca} .10^{-14} \mathrm{~S} \mathrm{~cm}^{-1}\right)$ results in low rate capability. ${ }^{178}$ Zeng et al. synthesized a hybrid nanorod array of Ti doped $\alpha$ $\mathrm{Fe}_{2} \mathrm{O}_{3}$ with poly(3,4-ethylenedioxythiophene) termed as $\mathrm{Ti}-\mathrm{Fe}_{2}-$ $\mathrm{O}_{3}$ @PEDOT on carbon cloth as an anode material and $\mathrm{MnO}_{2}$ as

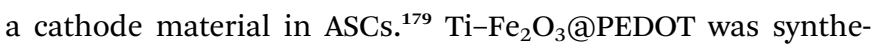
sized in two successive steps. Firstly Ti doped $\mathrm{Fe}_{2} \mathrm{O}_{3}$ nanorods were grown on carbon cloth via a hydrothermal technique and then the polymer shell of PEDOT was electrochemically deposited on the nanorod surface. Ti doping on $\mathrm{Fe}_{2} \mathrm{O}_{3}$ (core) significantly increased the electron donating capacity of $\mathrm{Fe}_{2} \mathrm{O}_{3}$ and hence increased the capacitive performance of ASCs. On the other hand the conductive polymer PEDOT used as the shell enhanced the conductivity and prevented the degradation of architectures as well. The authors found that the core-shell architecture offers an excellent capacitive performance of $1.15 \mathrm{~F}$ $\mathrm{cm}^{-2}\left(311.6 \mathrm{~F} \mathrm{~g}^{-1}\right.$ and $28.8 \mathrm{~F} \mathrm{~cm}^{-3}$ at $\left.1 \mathrm{~mA} \mathrm{~cm}^{-2}\right)$ and maximum

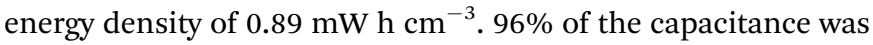
found to be retained after 30000 cycles. 


\section{Flexible micro-supercapacitors}

Due to the increasing demand for miniaturized, flexible, light weight and wearable electronic devices it is a challenge to design and fabricate an energy storage device which is compatible with these requirements. Flexible and thin film SCs are attracting great attention in the field of light weight and wearable ultrathin energy management devices due to their higher power density than batteries, long cycling stability and environment-friendly nature. Technologies to develop flexible MSCs for real world applications are scarce. Various approaches have been investigated for the fabrication of flexible electrodes. Mainly two of these techniques involve fabrication of electrodes either by the direct growth of a CNPs/hybrid material on flexible, porous and light-weight substrates or by coating/ printing. ${ }^{28,41,59,72,83,115,180}$ For example Zhu et al. reported the fabrication of printable 3D MSCs based on nano-composites consisting of cobalt oxide (CoO) nanoflowers woven with CNT networks. ${ }^{180}$ A hydrothermal technique was used to synthesize $\mathrm{CoO} / \mathrm{CNT}$ nano-composites. A schematic representation of the steps involved in the growth of nano-composites is illustrated in Fig. 18(a) and the SEM image in Fig. 18(c). Further, an electrode for a flexible MSC device was fabricated on a PET surface in two steps (Fig. 18(b)). Firstly silver ink was screen printed on the PET surface followed by the printing of nano-composites as the active material. Evolution of the bending angle $v s$. capacitance ratio is shown in Fig. 18(d).

An attractive approach using a facile LBL based patterning technique for the fabrication of fully interdigitated 3D microscale energy storage devices inside a bulk volume of aerogel was demonstrated by Nyström et $a .^{36}$ Aerogels, with the highest known specific surface area and pore volume, can be fabricated from a variety of organic and inorganic materials. ${ }^{181}$ Negatively charged cross-linked cellulose nanofibril aerogels were used as a substrate material for the fabrication of MSCs and batteries by taking advantage of the coulombic attraction in LBL deposition as demonstrated in Fig. 19. For the fabrication of MSCs, cationic polyethyleneimine (PEI) aerogel was applied as a substrate and carboxylic acid functionalized SWCNTs as the electrode material (Fig. 19(b) and (c)). A multilayer (30 layer) film of PEI/polyacrylic acid was applied as a separator. The 3D SC performance was maintained even after $75 \%$ compressions and 400 cycles with a capacitance of $25 \mathrm{~F} \mathrm{~g}^{-1}$ (Fig. 19(d) and (e)). ${ }^{36}$

Qin et al. fabricated an ultrathin, flexible SSC of $26 \mu \mathrm{m}$ thickness, thinner than the commercial A4 print paper (almost $100 \mu \mathrm{m}) .{ }^{182}$ Chemical vapor deposition was used to prepare porous carbon nanofibers/graphite (pCNFs/G) hybrid films over a $\mathrm{Cu}$ foil supported Co catalyst. All the key steps involved in the fabrication of interdigitated electrodes are demonstrated in Fig. 20. The authors showed that thin film of porous graphite

(a)

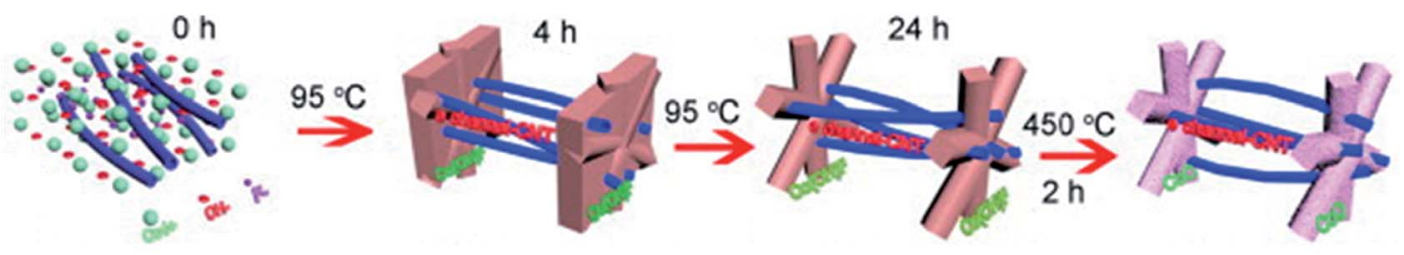

(b)

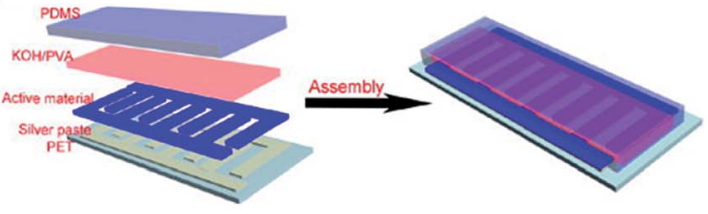

(d)
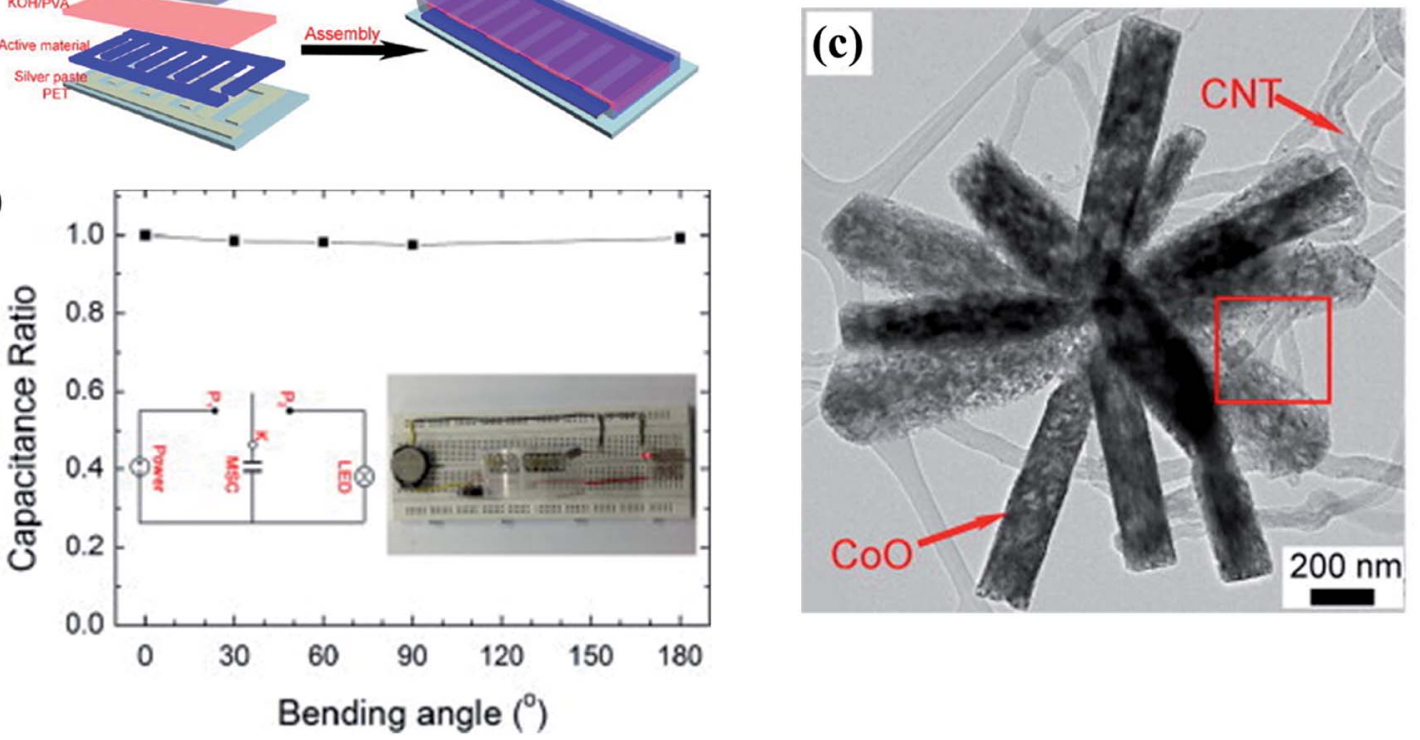

Fig. 18 (a) Growth mechanism of the CoO/CNTs. (b) Schematic view of the flexible printed MSC. (c) Low magnification TEM image of CoO nanoflowers woven by CNTs. (d) Evolution of the capacitance ratio vs. bending angle; insets show the digital photograph of the MSC that powers commercialized LED and the corresponding circuit diagram. ${ }^{180}$ Adopted and modified from ref. 180 with permission from Elsevier, 2013. 

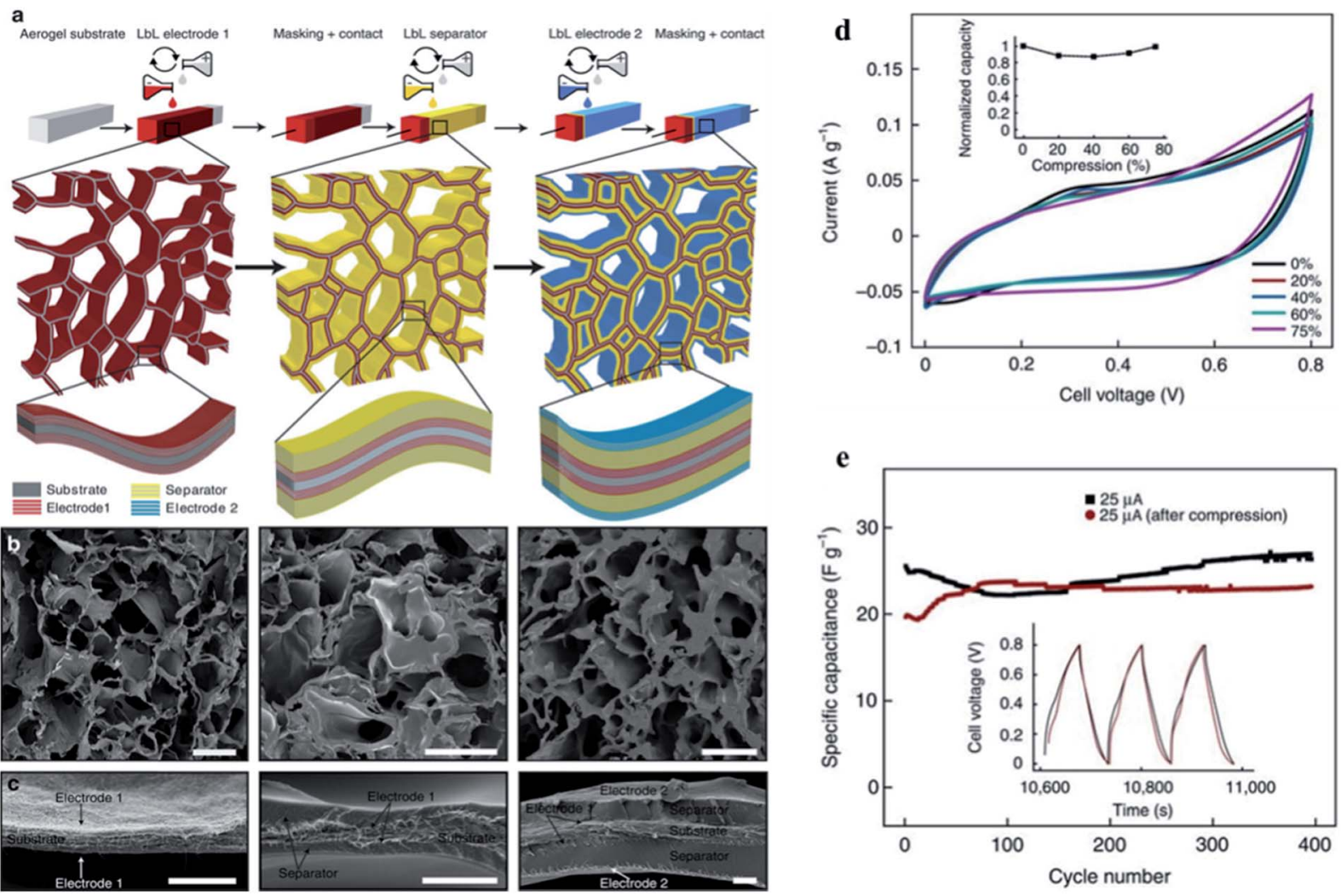

Fig. 19 Device assembly and structural architecture. (a) Schematics of the LBL process used to assemble 3D devices in an aerogel. (b and c) Cross-sectional SEM images of the first PEI/CNT electrode (left column), the PEI/CNT electrode with the separator (middle column) and the full device (right column). Scale bars: (b) $50 \mu \mathrm{m}$ and (c) $2 \mu \mathrm{m}$. (d) Cyclic voltammetry (CV) performed in situ during compression at a scan rate of 10 $\mathrm{mV} \mathrm{s}{ }^{-1}$ with corresponding calculated normalized charge capacities (inset). (e) Comparison of the galvanostatic curves before and after compression of the device (inset) and cycle life data before and after compression. Aqueous $1 \mathrm{M} \mathrm{Na}_{2} \mathrm{SO}_{4} \mathrm{pH}_{7}$ was used as the electrolyte. ${ }^{36}$ Adopted and modified from ref. 36 with permission from Macmillan Publishers Limited.

$(\sim 25 \mathrm{~nm})$ was also formed and worked as a current collector even after removing the $\mathrm{Cu}$ foil. Due to the good ohmic contact between the pCNFs and graphite sheet, electrons and ions can be transported easily through the pCNFs, which offers high power density to the device. The maximum energy density obtained was $2.4 \mathrm{~mW} \mathrm{~h} \mathrm{~cm}^{-3}$ and power density was $23 \mathrm{~W} \mathrm{~cm}^{-3}$ with the PVA- $\mathrm{H}_{2} \mathrm{SO}_{4}$ gel electrolyte. The largest specific capacitance of the $\mathrm{pCNFs} / \mathrm{G}$ electrode was found to be $120 \mathrm{~F} \mathrm{~g}^{-1}$ at a current density of $1 \mathrm{~A} \mathrm{~g}^{-1}$. The cyclic stability of the device showed $96 \%$ retention after 5000 cycles at a current density of

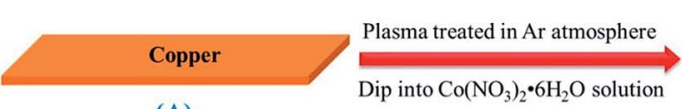

(A)

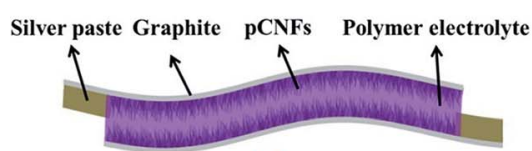

(F)

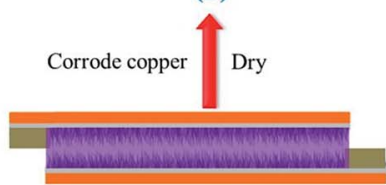

(E)

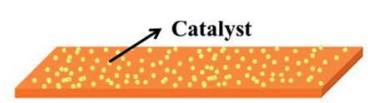

(B)

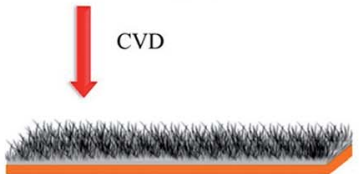

(C)

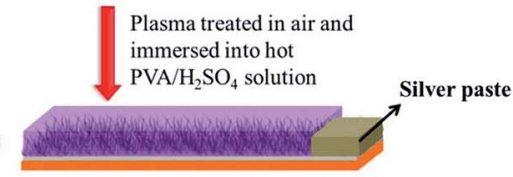

(D)

Fig. 20 Schematic diagram of the fabrication process of flexible solid-state SCs based on pCNFs/G hybrid films. ${ }^{182}$ Adopted from ref. 182 with permission from the American Chemical Society. 
$5 \mathrm{~A} \mathrm{~g}^{-1} \cdot{ }^{182} \mathrm{Kim}$ et al. fabricated a flexible MSC from a photoresist derived carbon by using simple transfer techniques. The areal capacitance was found to be $0.75 \mathrm{mF} \mathrm{cm}^{-2}$ at a scan rate of 100 $\mathrm{mV} \mathrm{s}^{-1}$ which remained at $0.36 \mathrm{mF} \mathrm{cm}^{-2}$ at a higher scan rate of $10 \mathrm{~V} \mathrm{~s}^{-1}$ at a potential window of $0-0.8 \mathrm{~V}$ using $0.5 \mathrm{M} \mathrm{H}_{2} \mathrm{SO}_{4}$ aqueous electrolyte. The cycling was performed for 10000 cycles at a scan rate of $100 \mathrm{mV} \mathrm{s}^{-1}$ and it showed $86 \%$ retention of the initial capacitance. The device also exhibited good flexibility and showed only $2 \%$ deviation of the initial volumetric capacitance after 300 cycles at a scan rate of $100 \mathrm{mV} \mathrm{s}^{-1}$ while the bending radius varied from $17.5 \mathrm{~mm}$ to $5 \mathrm{~mm} .{ }^{183}$

At the nanoscale, the contact resistance between two rGO layers efficiently affects the electrical transport properties of nanographene sheets. Much effort has been taken to reasonably design highly conductive graphene based electrode architectures for MSCs at an economic level. One such effort was attempted by Liu et al. ${ }^{\mathbf{1 8 4}}$ They synthesized a flexible interdigitated MSC electrode based on the composite film of graphene/ $\mathrm{MnO}_{2} / \mathrm{Ag}$ nanowires (RGMA) by using vacuum filtration followed by a thermal reduction technique. The morphology of
RGMA was needle-like having diameters ranging from 20 to 50 $\mathrm{nm}$ and length varying from 200 to $500 \mathrm{~nm}$ (Fig. 21). They showed that by increasing the interspace distance between electrode fingers from $200 \mu \mathrm{m}$ to $800 \mu \mathrm{m}$ the performance of the MSC decreased in terms of its volumetric capacitance and power density. Increasing the interspace distance increases the average ionic diffusion pathway resulting in increase of electrolyte resistance and hence an overall decrease in the MSC's performance. Further, the effect of finger electrode width was also investigated. On increasing the finger's width specific capacitance decreases, as the narrow finger maximizes the available area as well as shortens the ionic diffusion pathway. The cyclic voltammetry curve remained rectangular even at a high scan rate of $50 \mathrm{~V} \mathrm{~s}^{-1}$ for all the devices, suggesting the high power capability of RGMA MSCs. ${ }^{184}$

In a similar report, a simple vacuum filtration technique followed by thermal reduction of graphene oxide was used by Kim et al. to synthesize $\mathrm{rGO} / \mathrm{TiO}_{2}$ composite-free standing film for flexible MSCs (electrode area $0.1 \mathrm{~cm}^{2}$ ). ${ }^{185}$ The $\mathrm{rGO} / \mathrm{TiO}_{2}$ composite film was thermally reduced at $600{ }^{\circ} \mathrm{C}$ and
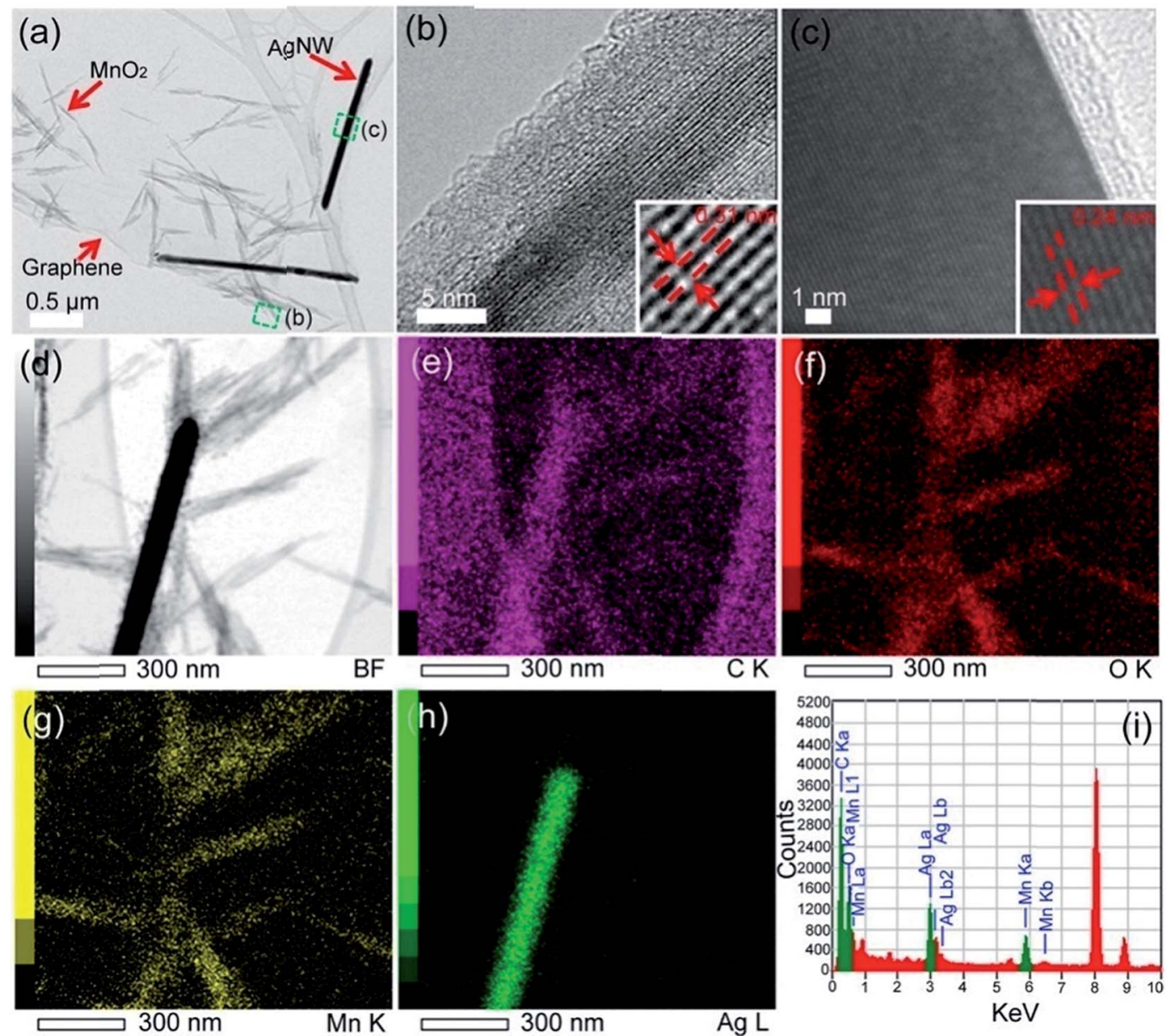

Fig. 21 TEM images of the RGMA ternary hybrid film: (a) low-magnification micrograph, (b) and (c) HR-TEM images of the open square regions (green line) located in panel (a), respectively, (d) HAADF-STEM image of the RGMA ternary hybrid film and the corresponding HAADF-STEM-EDS elemental mapping analysis of (e) C, (f) O, (g) Mn and (h) Ag, (i) EDS spectrum of the RGMA ternary hybrid film. ${ }^{184}$ Adopted from ref. 184 with permission from the American Chemical Society. 

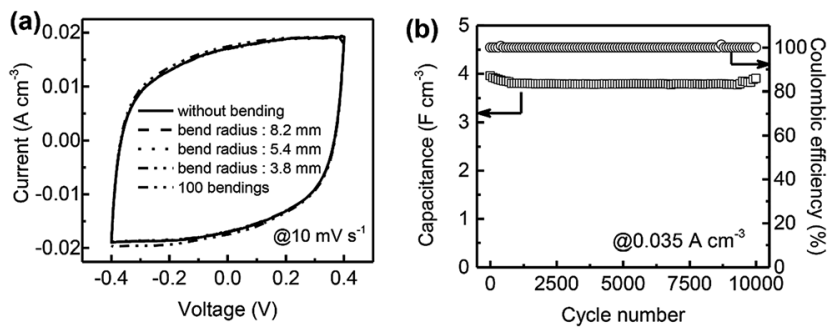

Fig. 22 (a) CV curves of MSC- 100 at $60^{\circ}, 90^{\circ}$, and $120^{\circ}$ bending angles (8.2, 5.4 and $3.8 \mathrm{~mm}$ of bending radii, respectively) and after 100 bending cycles (with a radius of curvature of $5.4 \mathrm{~mm}$ ) at a constant scan rate of $10 \mathrm{mV} \mathrm{s}^{-1}$. (b) Cycle stability and coulombic efficiency of MSC-100 during 10000 GCD cycles at $0.035 \mathrm{~A} \mathrm{~cm}^{-3}$. 188 Adopted and modified from ref. 188 with permission from WILEY-VCH Verlag $\mathrm{GmbH} \&$ Co. KGaA, Weinheim.

electrochemically characterized in $1 \mathrm{M} \mathrm{Na}_{2} \mathrm{SO}_{4}$ aqueous electrolyte at a potential window of $0-1 \mathrm{~V}$. The specific capacitance was found to be $286 \mathrm{~F} \mathrm{~g}^{-1}$ at a current density of $1 \mathrm{~A} \mathrm{~g}^{-1}$ with retention of $93 \%$ of the initial specific capacitance after 1000 GCD cycles at $1 \mathrm{~A} \mathrm{~g}^{-1}$. Peng et al. synthesized laser induced graphene from polyimide films for fabrication of flexible and stackable MSCs and the maximum specific capacitance obtained was $9.11 \mathrm{mF} \mathrm{cm}^{-2}$ at a current density of $0.02 \mathrm{~mA} \mathrm{~cm}^{-2}$ in solid PVA- $\mathrm{H}_{2} \mathrm{SO}_{4}$ electrolyte. ${ }^{186}$ The as-prepared SC retained its specific capacitance regardless of the bending radius. A flexible photo-detector system integrated with MSCs based on rGO electrodes was proposed by Shen $e t$ al. Photolithography technique was used to fabricate rGO electrodes and it showed a volumetric capacitance of $8 \mathrm{~F} \mathrm{~cm}^{-3}$ along with an energy density of $6.2 \mathrm{~W} \mathrm{~h} \mathrm{~cm}^{-3}$. MSCs were used as the power source for CdS nanowire based photo-detectors having current on/off ratios of 34.5 and $79.8 .^{187}$

Similar to graphene, CNTs also possess key characteristics such as good mechanical properties, low density, high porosity and small cross-section area, and have been largely utilized in flexible MSCs as active materials. For instance, Kim et al. fabricated a flexible interdigitated finger electrode MSC with MWCNTs patterned on a poly(dimethylsiloxane) (PDMS) substrate with $\mathrm{PVA}-\mathrm{H}_{3} \mathrm{PO}_{4}$ gel electrolyte. The maximum volumetric capacitance was $5.5 \mathrm{~F} \mathrm{~cm}^{-3}$ at a scan rate of $10 \mathrm{mV} \mathrm{s}^{-1}$ at a potential window of $-0.4 \mathrm{~V}$ to $0.4 \mathrm{~V}$ for $100 \mu \mathrm{m}$ interspace, higher than that for $500 \mu \mathrm{m}$ and $1000 \mu \mathrm{m}$ interspace MSCs. The $\mathrm{CV}$ curve remained the same even for a bending angle of $120^{\circ}$, and retained $98 \%$ of the initial capacitance value after 10000 cycles at a current density of $0.035 \mathrm{~A} \mathrm{~cm}^{-3}$ as illustrated in Fig. $22 .{ }^{188}$

Flexible MSCs suffer from few drawbacks which limit their practical applications in the real world. One such major drawback is air instability; a major decrease in capacitance (retention of $\sim 3 \%$ of the initial value) was observed after one month. Last year Kim et al. reported the fabrication of highly stable flexible MSCs even in air. They showed $80 \%$ retention of specific capacitance after 30000 cycles during GCD at a current density of $1.4 \mathrm{~A} \mathrm{~cm}^{-3}$, with good stability up to $90 \%$ of the initial specific capacitance even after 8 weeks under ambient air conditions. ${ }^{189}$
A MWCNT based interdigitated MSC was fabricated with a photolithography patterned ionogel electrolyte over a flexible PET substrate. ${ }^{189}$ Until now in most of the reported flexible MSCs stretchability of the SCs is due to the constituent materials and substrate, which greatly limit the applications of MSCs in flexible and wearable electronics. ${ }^{129,158,166}$ In this regard, integration of MSCs into a circuit holds great promise for circuit design. Hong et al. fabricated all solid state flexible MSCs over a specially designed PDMS island with $\mathrm{PVA}-\mathrm{H}_{3} \mathrm{PO}_{4}$ electrolyte by using MWCNT/ $\mathrm{Mn}_{3} \mathrm{O}_{4}$ as the electrode material. ${ }^{72}$ Electrical connections were made by a liquid metal Galinstan to connect active devices for the minimization of strain. The strain applied to the device was accommodated by the substrate material. On repeated deformation the overall performance of the MSCs was not affected much due to interconnections. The authors performed the CV test for 3000 cycles after stretching under $40 \%$ uniaxial strain at $200 \mathrm{mV} \mathrm{s}^{-1}$ scan rate and observed $92 \%$ retention of the initial specific capacitance (Fig. 23). The maximum areal capacitance for the single electrode was found to be $0.63 \mathrm{mF} \mathrm{cm}^{-2}$ at a scan rate of $10 \mathrm{mV} \mathrm{s}^{-1}$ at a potential window of $0-3 \mathrm{~V}$ and the corresponding energy density and power density were found to be $2.6 \mathrm{~mW} \mathrm{~h} \mathrm{~cm}{ }^{-3}$ and $23 \mathrm{~W} \mathrm{~cm}^{-3}$, respectively. Integration on both sides resulted in increase in the areal density by two times compared to planar MSCs. More significantly serial interconnection offered higher output voltage beyond the electrolyte limits. The authors also proposed that such MSCs hold great potential for future application in wireless, body-implantable and wearable nano-electronic devices without the need for any external power source.

Lim et al. reported the fabrication of biaxially stretchable interdigitated MSCs. They locally implanted polyethylene terephthalate (PET) film on a soft elastomer (Ecoflex) substrate as a new electrode material to achieve superior stretchability. The key steps and primary concept involved in the fabrication of MSCs are demonstrated in Fig. $24 .{ }^{190}$ Surface functionalized MWCNTs with $-\mathrm{COOH}$ and $-\mathrm{NH}_{2}$ groups were used as electrode materials and the LBL assembly was used to prepare interdigitated MSCs on flexible substrates using poly(ethylene glycol) diacrylate and 1-ethyl-3-methylimidazolium bis(trifluoromethylsulfonyl)imide as the ionogel electrolyte. Such fabricated MSCs demonstrated an energy density of $25 \mathrm{~mW} \mathrm{~h}$ $\mathrm{cm}^{-3}$ at a power density of $0.75 \mathrm{~W} \mathrm{~cm}^{-3}$ which remained at 11 $\mathrm{mW} \mathrm{h} \mathrm{cm}{ }^{-3}$ at a higher power density of $32.18 \mathrm{~W} \mathrm{~cm}^{-3}$ at the biaxial strain of $50 \%$ and showed that there is no noticeable change in the device performance after applying the strain. The authors tested the mechanical stability of those devices by applying $70 \%$ uniaxial stretching during the $\mathrm{CV}$ test which showed only $4 \%$ loss in capacitance after 5000 cycles. They also lighted $\mu$-LEDs with this device under stretching conditions and without stretching, and observed that there was no change in the brightness of $\mu$-LEDs even after stretching up to $50 \%{ }^{190}$

Dand et al. fabricated flexible wire shaped MSCs based on a $\mathrm{Ni}(\mathrm{OH})_{2}$ nanowire fiber electrode (Fig. 25) as the positive electrode and an ordered mesoporous carbon fiber electrode as the negative electrode with a PVA-KOH solid electrolyte. The length of the wire shaped MSC device was $3 \mathrm{~cm}$ and the diameter was $0.6 \mathrm{~mm}$. The length specific capacitance, areal 
a

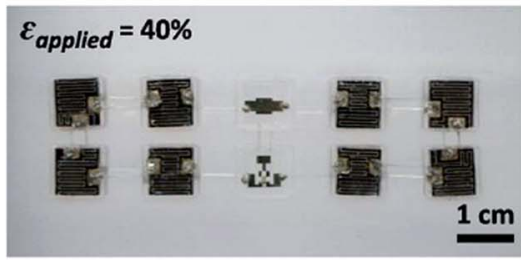

C

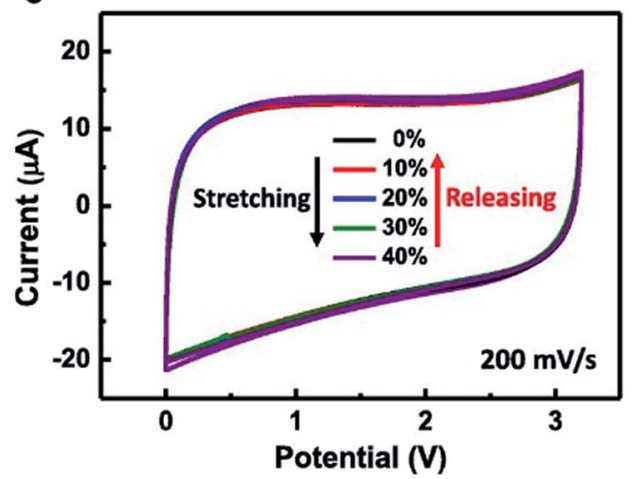

e

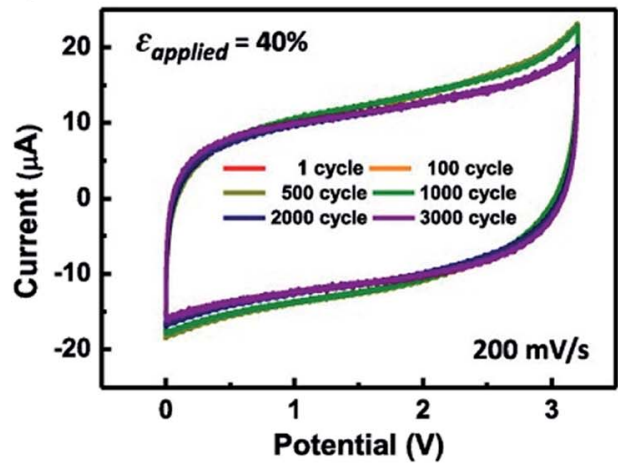

b

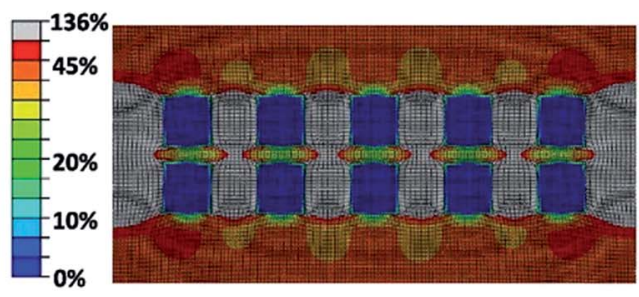

d

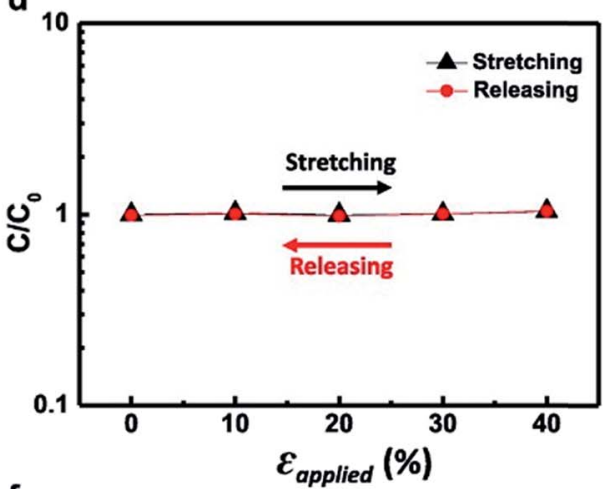

f

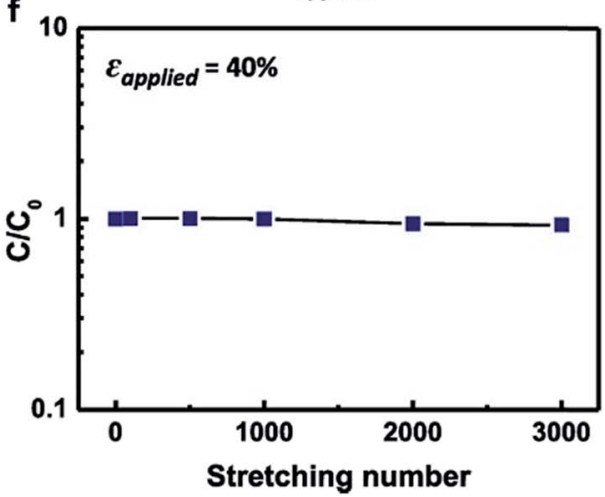

Fig. 23 (a) Photograph of an MSC array under an applied strain of 40\%; (b) distribution of the maximum principal strain estimated using the finite element method (FEM) upon uniaxial stretching at an applied strain of $40 \%$; (c) CV curves of the MSC array obtained while increasing the strain from $0 \%$ to $40 \%$ (stretching) and decreasing the strain from $40 \%$ to $0 \%$ (releasing); (d) normalized capacitance $\left(C / C_{0}\right)$ of the MSC array with the strain varying between $0 \%$ and $40 \%$ : black triangles (stretching) and red circles (releasing); (e) CV curves of the MSC array subjected to repeated stretching under an applied strain of $40 \%$; ( $f$ ) normalized capacitance $\left(C / C_{0}\right)$ as a function of the number of stretching cycles under a strain of $40 \%$. $C$ and $C_{0}$ are the capacitances after and before stretching, respectively. ${ }^{72}$ Adopted from ref. 72 with permission from the American Chemical Society.

capacitance and mass specific capacitance were found to be $6.67 \mathrm{mF} \mathrm{cm}^{-1}, 35.67 \mathrm{mF} \mathrm{cm}^{-2}$ and $32 \mathrm{~F} \mathrm{~g}^{-1}$, respectively at a current of $0.1 \mathrm{~mA}$ which remained at $4 \mathrm{mF} \mathrm{cm}{ }^{-1}, 21.22 \mathrm{mF}$ $\mathrm{cm}^{-2}$ and $119.2 \mathrm{~F} \mathrm{~g}^{-1}$ respectively at a high current of $1 \mathrm{~mA}$. The energy density was found to be $0.01 \mathrm{~mW} \mathrm{~h} \mathrm{~cm}^{-2}$ and $2.16 \mathrm{~mW} \mathrm{~h}$ $\mathrm{cm}^{-3}$ at a current of $0.1 \mathrm{~mA}$. Their device showed $70 \%$ retention over 10000 cycles at a current of $0.5 \mathrm{~mA}$ and was capable of lighting a red LED for $40 \mathrm{~s}$ after being charged at $3 \mathrm{~V}$ for $80 \mathrm{~s}$ as demonstrated in Fig. 26. ${ }^{\mathbf{1 9 1}}$

Wearable electronics are continuously attracting attention in modern technologies, and demand further development of compatible microscale energy storage devices that can be woven into cloths. ${ }^{192}$ Fabrication of wire/fiber shaped energy storage devices such as MSCs is the key requirement to be used as energy sources in wearable electronics. Wire MSCs offer promising applications with high flexibility and lightweight structures. Since first reported by Dalton and co-workers, ${ }^{193}$ CNTs and graphene hold great potential as electrode materials in fiber MSCs because they can be easily spun into fibers. ${ }^{\mathbf{1 9 4 , 1 9 5}}$ Enormous effort has been made for the development of lightweight, highly efficient and flexible wire SCs. However, there is still room for lot of improvement in their electrochemical performance.

Ma et al. fabricated composite fibers of GO and pristine fewwalled CNTs (FWCNTs) by dispersing FWCNTs in GO suspension and then spinning them into fibers using a Petri dish wetspinning approach. GO-FWCNT fibers were then reduced with HI to obtain rGO-FWCNT composite fibers. ${ }^{196}$ FWCNTs were used as a filler due to the higher electrical conductivity than MWCNTs and lower aggregation than SWCNTs in order to increase the strength and flexibility of the fibers. Flexible two-ply wearable SCs were assembled by using composite fibers of rGO- 
a

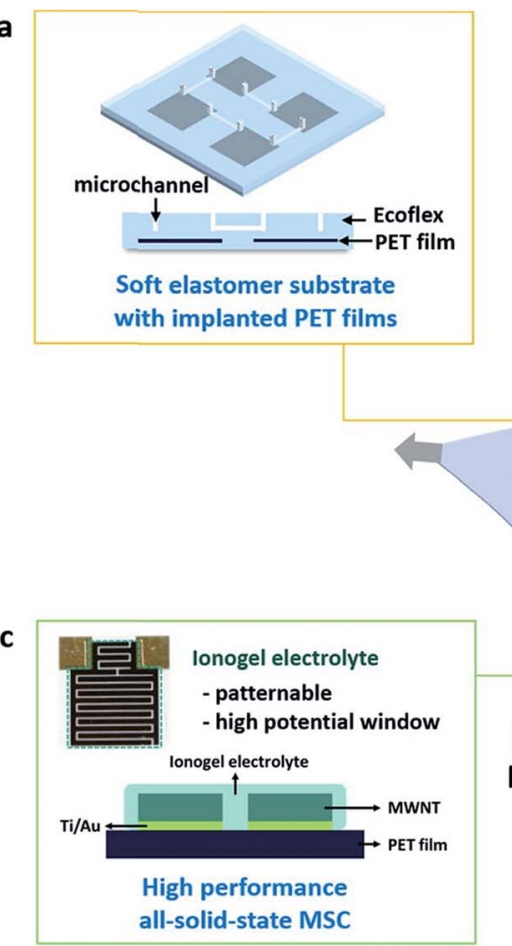

b

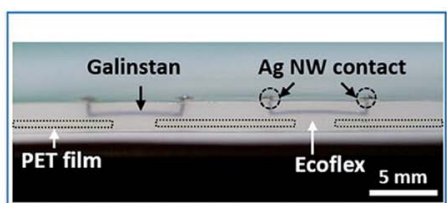

Embedded liquid metal interconnection

Fig. 24 Primary design concept of biaxially stretchable MSC array on a deformable substrate. (a) Structure of the deformable substrate, composed of Ecoflex with implanted PET films and an embedded microchannel for liquid metal interconnection. (b) Cross-sectional image of liquid metal interconnection embedded within the substrate and sealed with a Ag NW contact. (c) Optical image (top) and cross-sectional scheme (bottom) of the high-performance all-solid-state MSC with an ionogel electrolyte. (d) MSC array attached on the deformable substrate via a dry-transfer method. ${ }^{190}$ Adopted from ref. 190 with permission from the American Chemical Society, 2014.

FWCNTs as electrodes in the solid electrolyte of $\mathrm{PVA}-\mathrm{H}_{3} \mathrm{PO}_{4}$ gel. Such fabricated fiber SCs exhibited good charge/discharge capability of $3.4 \mathrm{~mW} \mathrm{~h} \mathrm{~cm} \mathrm{~cm}^{-3}$ with a volume capacitance of $38.8 \mathrm{~F}$ $\mathrm{cm}^{-3}$. The authors increased the linear capacitance by twisting many composite fibers to resolve the issue of performance degradation while increasing the fiber length.

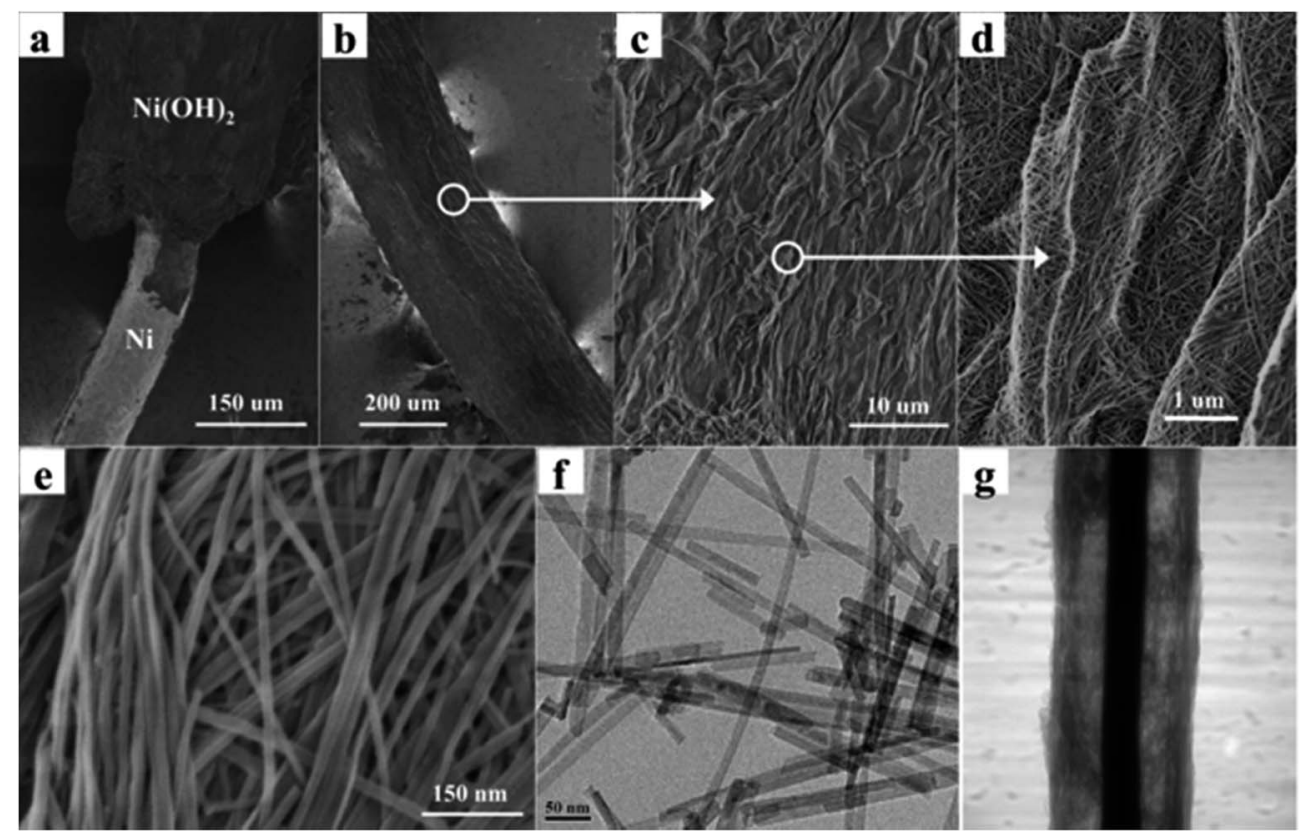

Fig. 25 Microscopy measurements of a Ni(OH)$)_{2}$ - nanowire fiber-electrode. (a-e) SEM images with different magnifications, (f) TEM image, (g) TXM image. ${ }^{191}$ Adopted from ref. 191 with permission from WILEY-VCH Verlag GmbH \& Co. KGaA, Weinheim. 

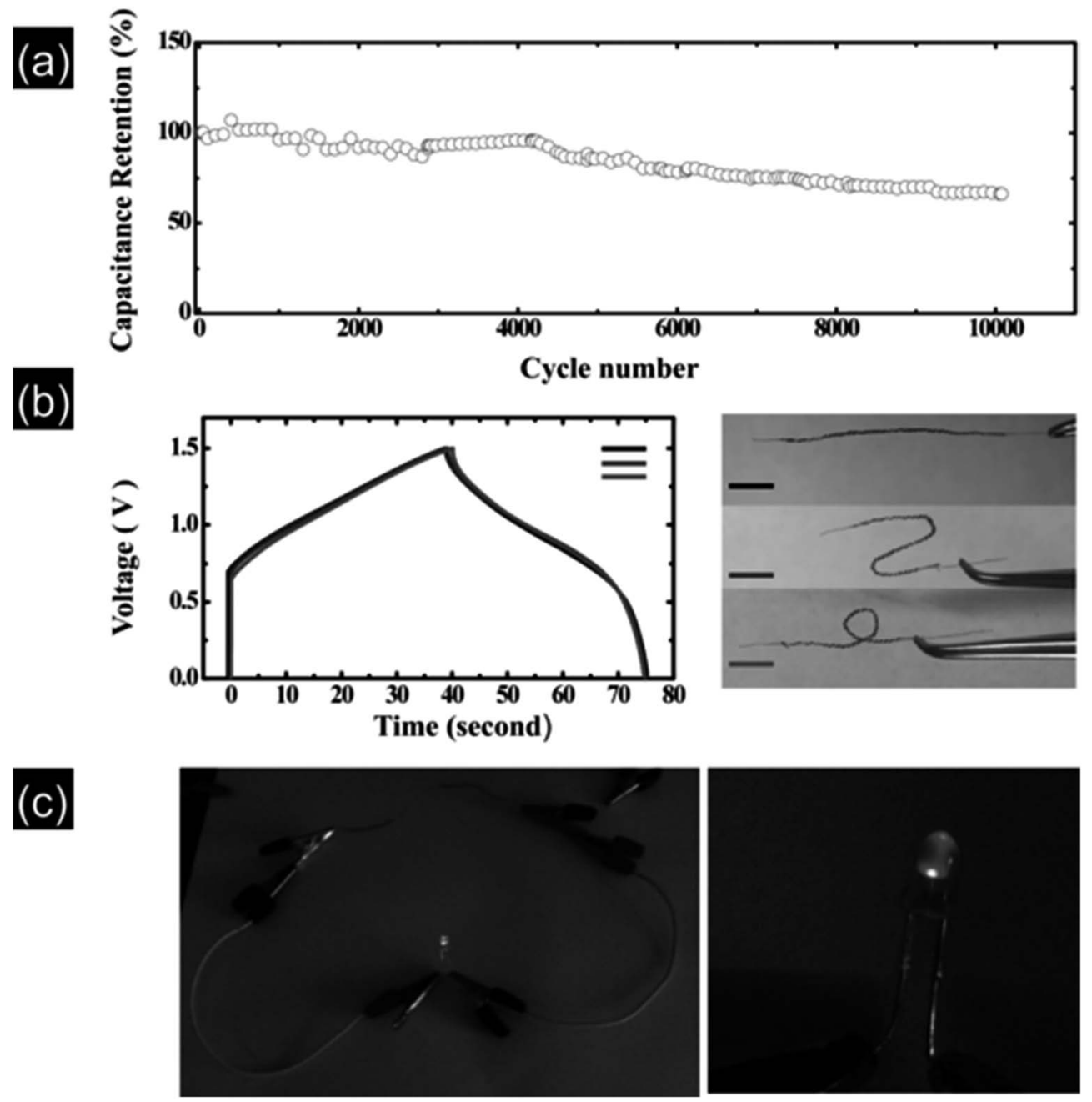

Fig. 26 Stability and demo application of wire-shaped micro-SCs. (a) Cyclic performance (investigated at an applied current of $0.5 \mathrm{~mA}$ within the voltage window from $0 \mathrm{~V}$ to $1.5 \mathrm{~V}$ ); (b) charge/discharge curves at different bending states; (c) digital photo that shows two micro-devices in series to light a LED. ${ }^{191}$ Adopted from ref. 191 with permission from WILEY-VCH Verlag GmbH \& Co. KGaA, Weinheim.

Dai et al. fabricated highly flexible and portable solid state MSCs with the use of nanostructured pen ink-carbon-fiber (Ink$\mathrm{CF})$ as an active electrode material. ${ }^{197}$ Commercially available carbon fiber thread was used as a scaffold and current collector, and was repeatedly coated with pen ink till the coating reached a mass density of $0.48 \mathrm{mg} \mathrm{cm}^{-1}$ by using the dip-coating process. Ink coating was used to increase the conductivity of CF without altering the shape. Fabrication of wire shaped MSCs is demonstrated in Fig. 27(a). Initially a plastic wire was coated with CF and then LiCl-PVA solid-state gel electrolyte. CV curves of both coated and uncoated CF are demonstrated in Fig. 27(b) and the MSCs in Fig. 27(c). The specific capacitance of both the MSCs (CF and Ink-CF) is demonstrated in Fig. 27(d), which clearly reveals the higher specific capacitance of the coated MSCs $\left(4.31 \mathrm{mF} \mathrm{cm}^{-2}\right)$ in contrast to bare MSCs $\left(0.81 \mathrm{mF} \mathrm{cm}^{-2}\right)$. GCD curves of the Ink-CF MSCs is shown in Fig. 27(e). The energy density was found to be $3.8 \times 10^{-7} \mathrm{~W} \mathrm{~h} \mathrm{~cm}^{-2}$ at a power density of $5.6 \times 10^{-6} \mathrm{~W} \mathrm{~cm}^{-2}$ for such fabricated wire MSCs. To demonstrate the potential application of MSCs in self-powered micro/nanosystems, the authors integrated these MSCs with a triboelectric nanogenerator and charged 8 light-emittingdiodes (LEDs). Fig. 27(f) demonstrates the schematic diagram of integration of MSCs with the triboelectric nanogenerator and the optical image for the same is shown in Fig. 27(g). Lighting of LEDs and the corresponding charging curve are shown in Fig. 27(h) and (i), respectively.

Liu et al. proposed an economic and green technique for the fabrication of wire SCs with high potential electrochemical performance. ${ }^{198}$ They fabricated a nanocube array of $\mathrm{Mn}_{2} \mathrm{O}_{3}$ which was directly aligned on carbon fibers via hydrothermal treatment. SCs were fabricated on PET films with composite fibers in $\mathrm{PVA} / \mathrm{H}_{2} \mathrm{SO}_{4}$ gel electrolyte with different structures and design such as straight (S-FSC), bent (B-FSC) and coiled (C-FSC) as demonstrated in Fig. 28(a-c). The flexibility of the devices is demonstrated in Fig. 28(d-f). Electrochemical performances (CV) are shown in Fig. 28(h). They showed that coiled electrode architecture exhibited the best performance among other architectures. 

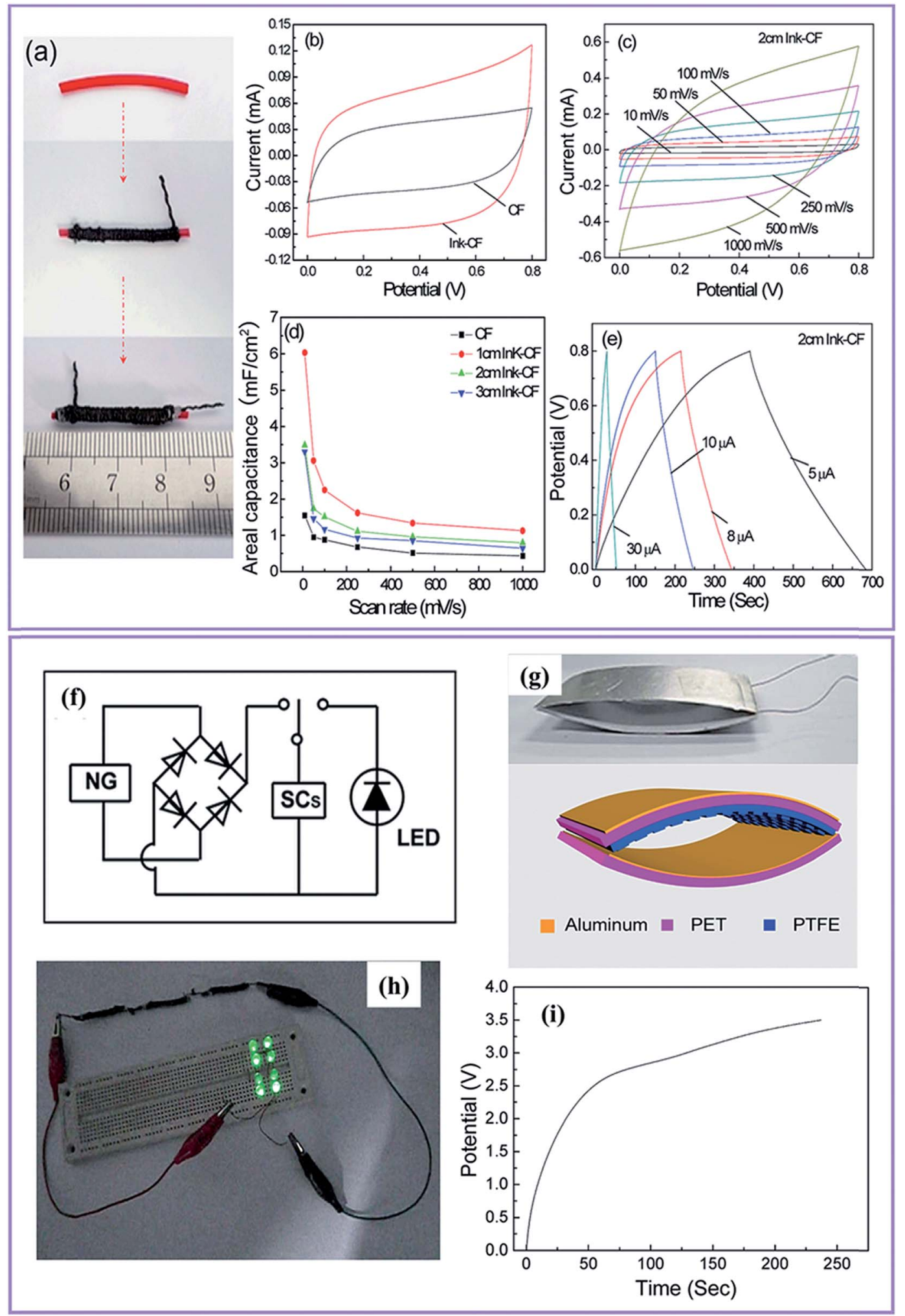

Fig. 27 (a) Optical photographs of the fabrication steps of the Ink-CF wire-shaped all-solid-state micro-supercapacitor, (b) CV curves of bare CF and Ink-CF thread devices at a scan rate of $100 \mathrm{mV} \mathrm{s}^{-1}$, (c) CV curves of the Ink-CF (about $2 \mathrm{~cm}$ ) thread device at different scan rates, (d) specific capacitances of the micro-supercapacitors with different structures at various scan rates, (e) galvanostatic charge-discharge curves of the InkCF thread device (about $2 \mathrm{~cm}$ ) at various currents, (f) schematic diagram of the self-powered nanosystem which is integrated by a triboelectric nanogenerator and three microsupercapacitors (2 cm each), (g) optical photograph (top) and schematic diagram of the triboelectric nanogenerator (bottom), (h) optical photograph of 8 commercialized LEDs lighted by charged microsupercapacitors connected in series, (i) charging curve of three micro-supercapacitors connected in series charged by using the triboelectric nanogenerator. ${ }^{197}$ Adopted and modified from ref. 197 with permission from The Royal Society of Chemistry. 
$\mathrm{Xu}$ et al. fabricated flexible wire shaped SCs based on a CNT fiber electrode in $\mathrm{H}_{2} \mathrm{SO}_{4}$-PVA gel electrolyte. ${ }^{199}$ Initially CNT fibers were coated with the gel electrolyte via dip coating and then twisted in a sinusoidal wave shape to fabricate wire shaped SCs. The schematic illustration of fabrication steps and optical images are demonstrated in Fig. 29. They demonstrated that up a

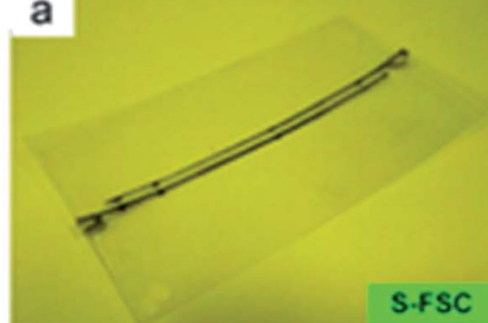

b

d

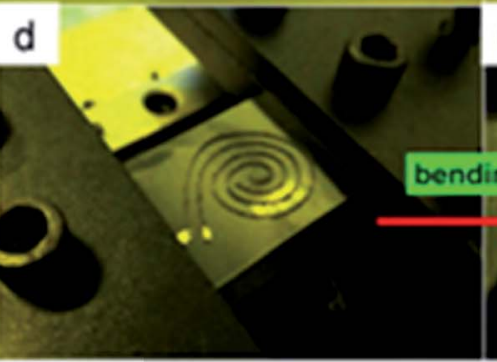

g

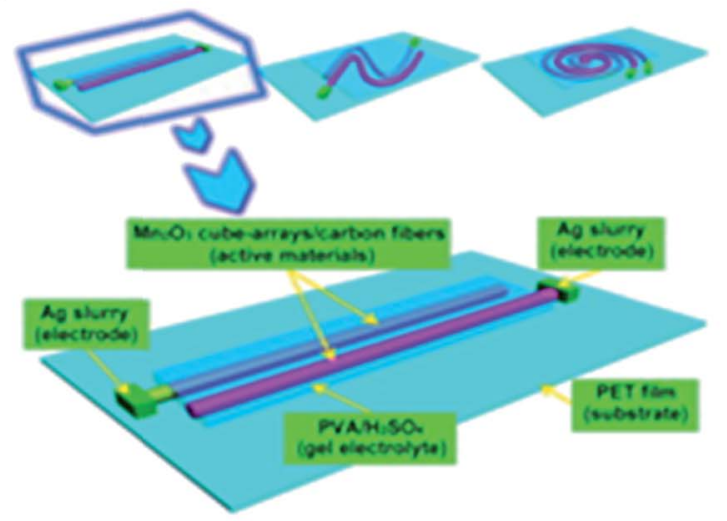

i

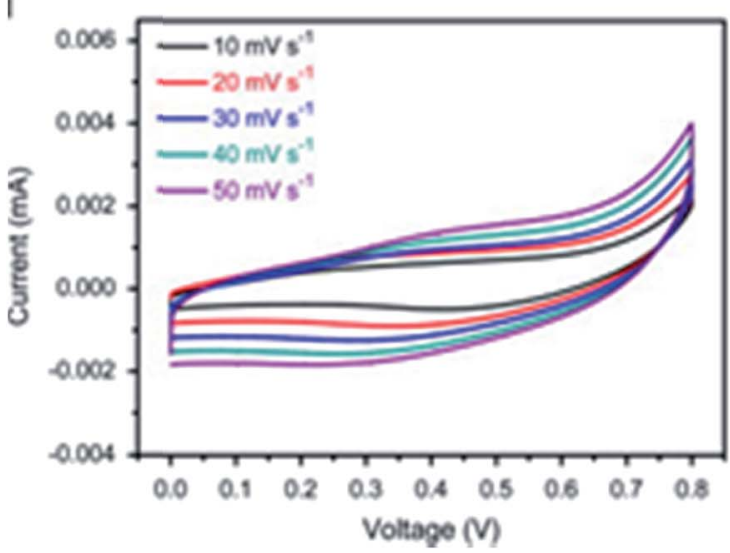

C<smiles>C=CC=C</smiles>

B.FSC

C-FSC
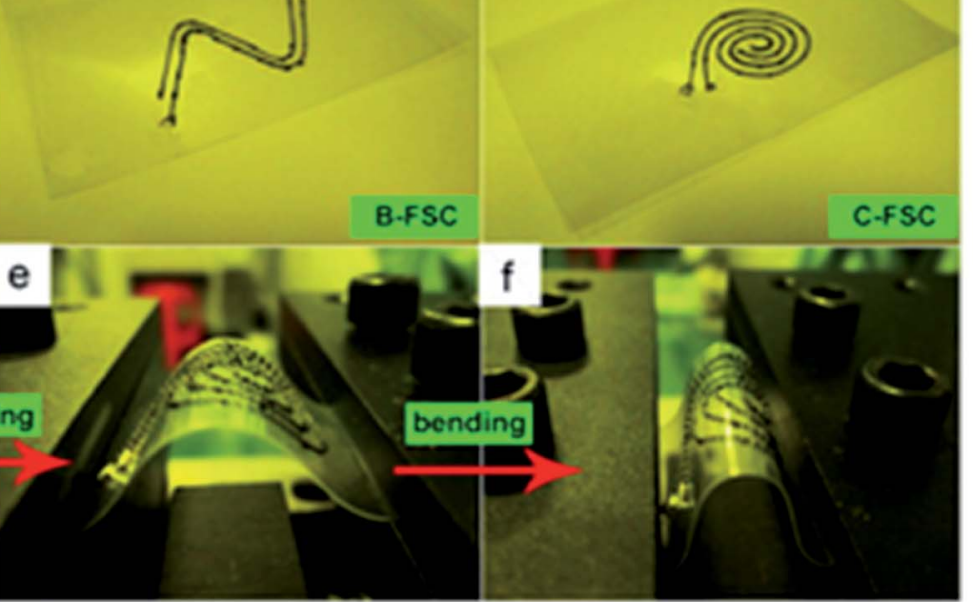

h

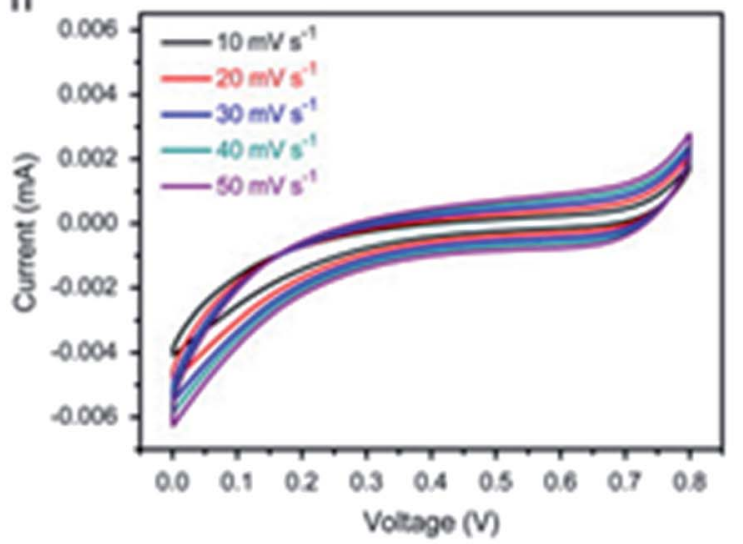

j

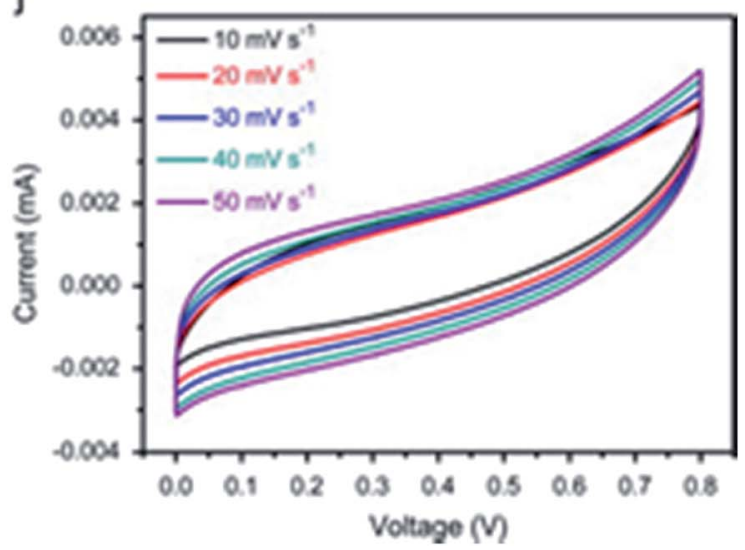

Fig. 28 Fiber-device demonstration and electrochemical characteristics of the as-fabricated fiber-supercapacitors measured at various scan rates between 0 and $0.8 \mathrm{~V}$. Photographs of (a), (b) and (c) three as-fabricated fiber-devices: S-FSC, B-FSC, and C-FSC respectively, and (d-f) bending process of a CFSC device. (g) Schematics of different architectures of the three devices, showing several components of the MSCs. Cyclic voltammetry of these devices based on $\mathrm{Mn}_{2} \mathrm{O}_{3}$ cube-arrays/carbon fiber electrodes measured between 0 and $0.8 \mathrm{~V}$ at various scan rates. Three mini-type fiber-supercapacitors: (h) S-FSC, (i) B-FSC, and (j) C-FSC. ${ }^{198}$ Adopted from ref. 198 with permission from Elsevier Ltd. 


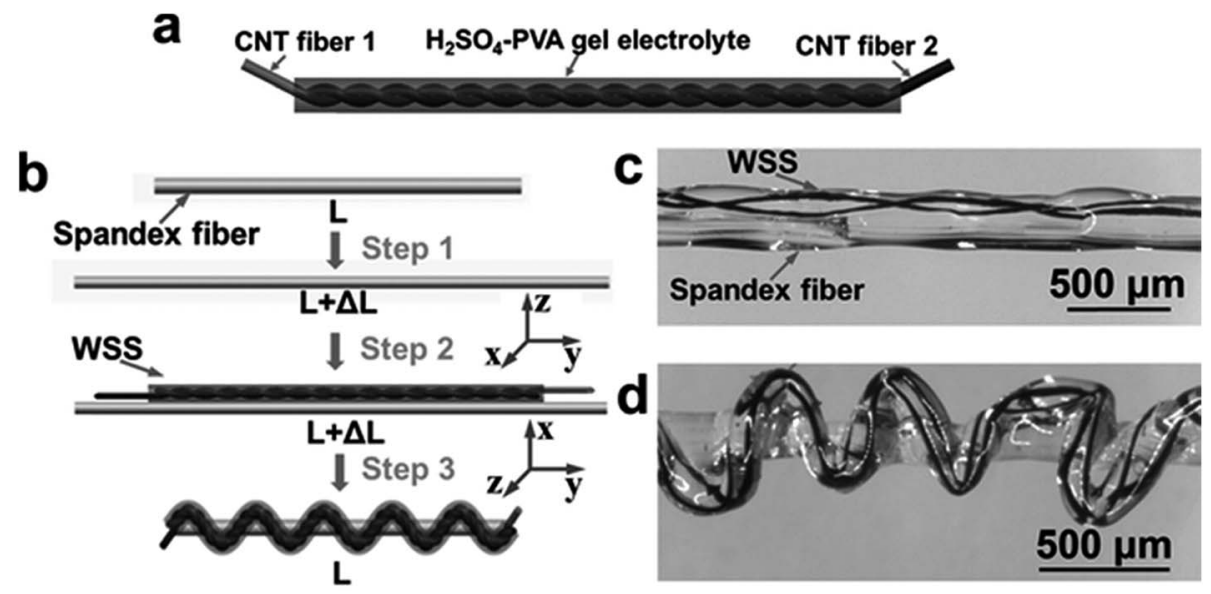

Fig. 29 (a) The structure of wire-shaped supercapacitors WSS and (b) schematics of the fabrication procedures for stretchable WSS. Optical microscopy images of (c) straight WSS combined with prestrained spandex fiber and (d) the buckled WSS with relaxed spandex fiber. ${ }^{199}$ Adopted from ref. 199 with permission from WILEY-VCH Verlag GmbH \& Co. KGaA, Weinheim.

to $100 \%$ strain or 20 successive loading unloading cycles, no significant reduction in the electrochemical performance was observed. More significantly $8.2 \%$ increase in areal specific capacitance and $25.8-55 \%$ increase in power density were observed after strain.

\section{Summary and perspectives}

This review summarized the recent research advancements in the fabrication techniques, electrode materials and performance of interdigitated miniaturized SCs and their practical integration in on-chip devices. Significant advances have been made in the miniaturization of SC devices and introducing flexibility among them. By controlling the composition of the electrode material/electrolytes and optimizing the electrode pattern, the performance of the MSCs can be improved. Although great achievements have already been made for the fabrication of flexible MSCs, there still exist many drawbacks which need to be addressed for enhancement in the performance and cycle life of MSCs. Furthermore, new generation transparent, flexible and multifunctional miniaturized SCs have been fabricated based on hierarchical nanomaterials and their composites with superior performance. Although some of the reports have demonstrated the fabrication and testing of interdigitated electrodes and further on-chip integration, fabrication and real word application of fully integrated and flexible SCs with "true" 3D architecture are still at an infant stage. The energy densities of MSCs are still very low compared to micro-batteries. Usage of aqueous based gel electrolytes is one of the limiting factors in current MSCs which limits the working voltage of the device. Immediate attention is required for the development of gel based solid electrolytes that improve the cell voltage. In this context, utilization of an ionic liquid or organic based polymer electrolyte may be an effective methodology in order to achieve high energy as well as high power densities of MSCs. Further, the combination of MSCs and micro-batteries may be another way to achieve higher energy and power densities.

The following research aspects need to be explored in order to introduce flexibility, on-chip integration and further improved performance.

(i) One of the major issues for the fabrication of flexible MSCs is the exploration of suitable current collectors which exhibit high flexibility, are economic in nature and easy to fabricate with good stability.

(ii) Fabrication of MSCs having real 3D characteristics with higher flexibility and energy density.

(iii) Practical integration and further utilization of miniature energy storage devices in real world applications are scarce. Hence, integration of miniaturized MSCs with other energy storage devices at the microscale needs to be further investigated.

(iv) Fabrication of eco-friendly wire MSCs and further enhancement of their electrochemical performances for wearable electronics.

Moreover, there is great hope that integration of green materials such as biomaterials and new functions could further expand their applications for the fabrication of green MSCs.

\section{Acknowledgements}

This work is supported by the Department of Atomic Energy (DAE), BRNS, India (Project No. 34/14/14/2014-BRNS) and Department of Science and Technology (DST), India INSPIRE Faculty Award (Project No. IFA-13 ENG-57).

\section{References}

1 D. Sinton, Lab Chip, 2014, 14, 3127-3134.

2 P. Simon and Y. Gogotsi, Philos. Trans. R. Soc., A, 2010, 368, 3457-3467.

3 P. Simon and Y. Gogotsi, Nat. Mater., 2008, 7, 845-854. 
4 L. L. Zhang and X. S. Zhao, Chem. Soc. Rev., 2009, 38, 25202531.

5 B. Conway, Scientific Fundamentals and Technological Applications, Kluwer Academic/Plenum Publishers, New York, 1999.

6 H. I. Becker, Google Patents, US2800616 A, 1957.

7 G. Xiong, C. Meng, R. G. Reifenberger, P. P. Irazoqui and T. S. Fisher, Electroanalysis, 2014, 26, 30-51.

8 R. Kötz and M. Carlen, Electrochim. Acta, 2000, 45, 24832498.

9 D. L. Boos, Google Patents, US3536963 A, 1970.

10 B. E. Conway, J. Electrochem. Soc., 1991, 138, 1539-1548.

11 N. S. A. Manaf, M. S. A. Bistamam and M. A. Azam, ECS J. Solid State Sci. Technol., 2013, 2, M3101-M3119.

12 P. Yang and W. Mai, Nano Energy, 2014, 8, 274-290.

13 G. Yu, X. Xie, L. Pan, Z. Bao and Y. Cui, Nano Energy, 2013, 2, 213-234.

14 Y. Zhang, H. Feng, X. Wu, L. Wang, A. Zhang, T. Xia, H. Dong, X. Li and L. Zhang, Int. J. Hydrogen Energy, 2009, 34, 4889-4899.

15 A. G. Pandolfo and A. F. Hollenkamp, J. Power Sources, 2006, 157, 11-27.

16 H. Liu and G. Zhu, J. Power Sources, 2007, 171, 1054-1061. 17 E. Raymundo-Piñero, K. Kierzek, J. Machnikowski and F. Béguin, Carbon, 2006, 44, 2498-2507.

18 M. J. Bleda-Martínez, J. A. Maciá-Agulló, D. Lozano-Castelló, E. Morallón, D. Cazorla-Amorós and A. Linares-Solano, Carbon, 2005, 43, 2677-2684.

19 M. Nakamura, M. Nakanishi and K. Yamamoto, J. Power Sources, 1996, 60, 225-231.

20 D. P. Dubal, J. G. Kim, Y. Kim, R. Holze, C. D. Lokhande and W. B. Kim, Energy Technol., 2014, 2, 325-341.

21 L. L. Zhang and X. S. Zhao, Chem. Soc. Rev., 2009, 38, 25202531.

22 B. E. Conway, J. Electrochem. Soc., 1991, 138, 1539-1548.

23 G. Wang, L. Zhang and J. Zhang, Chem. Soc. Rev., 2012, 41, 797-828.

24 M. Toupin, T. Brousse and D. Bélanger, Chem. Mater., 2004, 16, 3184-3190.

25 M. F. El-Kady and R. B. Kaner, Nat. Commun., 2013, 4, 14751483.

26 Z.-S. Wu, K. Parvez, X. Feng and K. Müllen, Nat. Commun., 2013, 4, 8288-8293.

27 J. Chmiola, C. Largeot, P.-L. Taberna, P. Simon and Y. Gogotsi, Science, 2010, 328, 480-483.

28 K. Wang, W. Zou, B. Quan, A. Yu, H. Wu, P. Jiang and Z. Wei, Adv. Energy Mater., 2011, 1, 1068-1072.

29 Y. Fu, H. Wu, S. Ye, X. Cai, X. Yu, S. Hou, H. Kafafy and D. Zou, Energy Environ. Sci., 2013, 6, 805-812.

30 X. Lu, M. Yu, G. Wang, Y. Tong and Y. Li, Energy Environ. Sci., 2014, 7, 2160-2181.

31 C. Meng, J. Maeng, S. W. M. John and P. P. Irazoqui, Adv. Energy Mater., 2014, 4, 1301269-1301275.

32 Z.-S. Wu, K. Parvez, X. Feng and K. Mullen, J. Mater. Chem. A, 2014, 2, 8288-8293.

33 W. Si, C. Yan, Y. Chen, S. Oswald, L. Hana and O. G. Schmidt, Energy Environ. Sci., 2013, 6, 3218-3223.
34 F. Liu, A. Gutes, I. Laboriante, C. Carraro and R. Maboudian, Appl. Phys. Lett., 2011, 99, 112104-112106.

35 L. Peng, X. Peng, B. Liu, C. Wu, Y. Xie and G. Yu, Nano Lett., 2013, 13, 2151-2157.

36 G. Nyström, A. Marais, E. Karabulut, L. Wagberg, Y. Cui and M. M. Hamedi, Nat. Commun., 2015, 6, 7259.

37 L. T. Le, M. H. Ervin, H. Qiu, B. E. Fuchs and W. Y. Lee, Electrochem. Commun., 2011, 12, 355-358.

38 M. Beidaghi and Y. Gogotsi, Energy Environ. Sci., 2014, 7, 867-884.

39 W. Liu, X. Yan, J. Chen, Y. Feng and Q. Xue, Nanoscale, 2013, 5, 6053-6062.

40 B. Hsia, M. S. Kim, M. Vincent, C. Carraro and R. Maboudian, Carbon, 2013, 57, 395-400.

41 M. Xue, Z. Xie, L. Zhang, X. Ma, X. Wu, Y. Guo, W. Song, Z. Lib and T. Cao, Nanoscale, 2011, 3, 2703-2708.

42 G. Fiorentino, S. Vollebregt, F. D. Tichelaar, R. Ishihara and P. M. Sarro, IEEE 27th International Conference on Micro Electro Mechanical Systems (MEMS), 2014.

43 C. K. Subramaniam and G. Boopalan, Appl. Phys. A, 2014, 116, 887-891.

44 F. Xiao, S. Yang, Z. Zhang, H. Liu, J. Xiao, L. Wan, J. Luo, S. Wang and Y. Liu, Sci. Rep., 2015, 5, 9359-9366.

45 H. Gao and K. Lian, RSC Adv., 2014, 4, 33091-33113.

46 B. G. Choi, J. Hong, W. H. Hong, P. T. Hammond and H. Park, ACS Nano, 2011, 5, 7205-7213.

47 D. Yu, Q. Qian, L. Wei, W. Jiang, K. Goh, J. Wei, J. Zhang and Y. Chen, Chem. Soc. Rev., 2015, 44, 647-662.

48 S. Ferrari, M. Loveridge, S. D. Beattie, M. Jahn, R. J. Dashwood and R. Bhagat, J. Power Sources, 2015, 286, 25-46.

49 Y. Wang, B. Liu, Q. Li, S. Cartmell, S. Ferrara, Z. D. Deng and J. Xiao, J. Power Sources, 2015, 286, 330-345.

50 P. E. Delannoy, B. Riou, T. Brousse, J. le Bideau, D. Guyomard and B. Lestriez, J. Power Sources, 2015, 287, 261-268.

51 X. Wang, X. Lu, B. Liu, D. Chen, Y. Tong and G. Shen, Adv. Mater., 2014, 26, 4763-4782.

52 C. W. Lee, S.-D. Seo, H. K. Park, S. Park, H. J. Song, D.-W. Kim and K. S. Hong, Nanoscale, 2015, 7, 2790-2796.

53 A. S. Westover, D. Freudiger, Z. S. Gani, K. Share, L. Oakes, R. E. Carter and C. L. Pint, Nanoscale, 2015, 7, 98-103.

54 P. E. Delannoy, B. Riou, B. Lestriez, D. Guyomard, T. Brousse and J. le Bideau, J. Power Sources, 2015, 274, 1085-1090.

55 H. Liu, H.-M. Cho, Y. S. Meng and Q. Li, ACS Appl. Mater. Interfaces, 2014, 6, 9842-9849.

56 E. Eustache, P. Tilmant, L. Morgenroth, P. Roussel, G. Patriarche, D. Troadec, N. Rolland, T. Brousse and C. Lethien, Adv. Energy Mater., 2014, 4, 1301612-1301622.

57 C. Zhao, C. Wang, R. Gorkin Iii, S. Beirne, K. Shu and G. G. Wallace, Electrochem. Commun., 2014, 41, 20-23.

58 Y. Xu, I. Hennig, D. Freyberg, A. James Strudwick, M. Georg Schwab, T. Weitz and K. Chih-Pei Cha, J. Power Sources, 2014, 248, 483-488.

59 Y. Wang, Y. Shi, C. X. Zhao, J. I. Wong, X. W. Sun and H. Y. Yang, Nanotechnology, 2014, 25, 094010. 
60 S. Wang, N. Liu, J. Tao, C. Yang, W. Liu, Y. Shi, Y. Wang, J. Su, L. Li and Y. Gao, J. Mater. Chem. A, 2015, 3, 2407-2413.

61 H. Pang, Y. Zhang, W.-Y. Lai, Z. Hu and W. Huang, Nano Energy, 2015, 15, 303-312.

62 M. H. Ervin, L. T. le and W. Y. Lee, Electrochim. Acta, 2014, 147, 610-616.

63 H. Jung, C. Ve Cheah, N. Jeong and J. Lee, Appl. Phys. Lett., 2014, 105, 053902.

64 K. Chi, Z. Zhang, J. Xi, Y. Huang, F. Xiao, S. Wang and Y. Liu, ACS Appl. Mater. Interfaces, 2014, 6, 16312-16319.

65 S. Liu, J. Xie, H. Li, Y. Wang, H. Y. Yang, T. Zhu, S. Zhang, G. Cao and X. Zhao, J. Mater. Chem. A, 2014, 2, 18125-18131.

66 Y. N. Sudhakar, M. Selvakumar, D. K. Bhat and S. Senthil Kumar, RSC Adv., 2014, 4, 60039-60051.

67 J. P. Mensing, A. Wisitsoraat, D. Phokharatkul, T. Lomas and A. Tuantranont, Composites, Part B, 2015, 77, 93-99.

68 F. Pettersson, J. Keskinen, T. Remonen, L. von Hertzen, E. Jansson, K. Tappura, Y. Zhang, C. E. Wilén and R. Österbacka, J. Power Sources, 2014, 271, 298-304.

69 A. B. Dighe, D. P. Dubal and R. Holze, Z. Anorg. Allg. Chem., 2014, 640, 2852-2857.

70 H. Hu, K. Zhang, S. Li, S. Ji and C. Ye, J. Mater. Chem. A, 2014, 2, 20916-20922.

71 N. Kurra, M. K. Hota and H. N. Alshareef, Nano Energy, 2015, 13, 500-508.

72 J. Yeo, G. Kim, S. Hong, M. S. Kim, D. Kim, J. Lee, H. B. Lee, J. Kwon, Y. D. Suh, H. W. Kang, H. J. Sung, J.-H. Choi, W.-H. Hong, J. M. Ko, S.-H. Lee, S.-H. Choa and S. H. Ko, J. Power Sources, 2014, 246, 562-568.

73 H. Lee, S. Hong, J. Kwon, Y. D. Suh, J. Lee, H. Moon, J. Yeo and S. H. Ko, J. Mater. Chem. A, 2015, 3, 8339-8345.

74 L. Coustan, A. L. Comte, T. Brousse and F. Favier, Electrochim. Acta, 2015, 152, 520-529.

75 Y. Qiu, Y. Zhao, X. Yang, W. Li, Z. Wei, J. Xiao, S.-F. Leung, Q. Lin, H. Wu, Y. Zhang, Z. Fan and S. Yang, Nanoscale, 2014, 6, 3626-3631.

76 G. Sun, J. An, C. K. Chua, H. Pang, J. Zhang and P. Chen, Electrochem. Commun., 2015, 51, 33-36.

77 J. Thomas, P. Gangopadhyay, E. Araci, R. A. Norwood and N. Peyghambarian, Adv. Mater., 2011, 23, 4782-4787.

78 B. Duong, Z. Yu, P. Gangopadhyay, S. Seraphin, N. Peyghambarian and J. Thomas, Adv. Mater. Interfaces, 2014, 1, 1300014-1300018.

79 S. Wang, B. Hsia, C. Carraroa and R. Maboudian, J. Mater. Chem. A, 2014, 2, 7997-8002.

80 D. Chandra and S. Yang, Acc. Chem. Res., 2010, 43, 10801091.

81 Z. Ma, C. Jiang, X. Li, F. Ye and W. Yuan, J. Micromech. Microeng., 2013, 23, 095027.

82 L. Zhou, H. Cao, S. Zhu, L. Hou and C. Yuan, Green Chem., 2015, 17, 2373-2382.

83 D. Yu, K. Goh, H. Wang, L. Wei, W. Jiang, Q. Zhang, L. Dai and Y. Chen, Nat. Nanotechnol., 2014, 9, 555-562.

84 S. Jiang, T. Shi, Y. Gao, H. Long, S. Xi and Z. Tang, J. Micromech. Microeng., 2014, 24, 045001-045008.

85 Y. Song, R. Agrawal, Y. Hao, C. Chen and C. Wanga, ECS Trans., 2014, 61, 55-64.
86 P. J. Hall, M. Mirzaeian, S. I. Fletcher, F. B. Sillars, A. J. R. Rennie, G. O. Shitta-Bey, G. Wilson, A. Cruden and R. Carter, Energy Environ. Sci., 2010, 3, 1238-1251.

87 D. R. MacFarlane, M. Forsyth, P. C. Howlett, J. M. Pringle, J. Sun, G. Annat, W. Neil and E. I. Izgorodina, Acc. Chem. Res., 2007, 40, 1165-1173.

88 J. L. Bideau, L. Viau and A. Vioux, Chem. Soc. Rev., 2011, 40, 907-925.

89 E. Frackowiak and F. Béguin, Carbon, 2001, 39, 937-950.

90 R. Madhu, K. V. Sankar, S.-M. Chen and R. K. Selvan, RSC Adv., 2014, 4, 1225-1233.

91 Q. Zhang, J.-Q. Huang, W.-Z. Qian, Y.-Y. Zhang and F. Wei, Small, 2013, 9, 1237-1265.

92 H. Qian, A. R. Kucernak, E. S. Greenhalgh, A. Bismarck and M. S. P. Shaffer, ACS Appl. Mater. Interfaces, 2013, 5, 61136122.

93 M. E. Plonska-Brzezinska and L. Echegoyen, J. Mater. Chem. A, 2013, 1, 13703-13714.

94 W. Gao, N. Singh, L. Song, Z. Liu, A. L. M. Reddy, L. Ci, R. Vajtai, Q. Zhang, B. Wei and P. M. Ajayan, Nat. Nanotechnol., 2011, 6, 496-500.

95 J. L. Delgado, M. A. Herranz and N. Martin, J. Mater. Chem., 2008, 18, 1417-1426.

96 Q. Wu, Y. Xu, Z. Yao, A. Liu and G. Shi, ACS Nano, 2010, 4, 1963-1970.

97 K. Jost, C. R. Perez, J. K. McDonough, V. Presser, M. Heon, G. Dion and Y. Gogotsi, Energy Environ. Sci., 2011, 4, 50605067.

98 M. Zhi, C. Xiang, J. Li, M. Li and N. Wu, Nanoscale, 2013, 5, 72-88.

99 R. A. Fisher, M. R. Watt and W. Jud Ready, ECS J. Solid State Sci. Technol., 2013, 2, M3170-M3177.

100 A. Izadi-Najafabadi, T. Yamada, D. N. Futaba, M. Yudasaka, H. Takagi, H. Hatori, S. Iijima and K. Hata, ACS Nano, 2011, 5, 811-819.

101 P. Dubey, S. K. Sonkar, S. Majumder, K. M. Tripathi and S. Sarkar, RSC Adv., 2013, 3, 7306-7312.

102 S. K. Sonkar, K. M. Tripathi and S. Sarkar, J. Nanosci. Nanotechnol., 2014, 14, 2532-2538.

103 J. Chen, A. I. Minett, Y. Liu, C. Lynam, P. Sherrell, C. Wang and G. G. Wallace, Adv. Mater., 2008, 20, 566-570.

104 D. N. Futaba, K. Hata, T. Yamada, T. Hiraoka, Y. Hayamizu, Y. Kakudate, O. Tanaike, H. Hatori, M. Yumura and S. Iijima, Nat. Mater., 2006, 5, 987-994.

105 J. Lin, C. Zhang, Z. Yan, Y. Zhu, Z. Peng, R. H. Hauge, D. Natelson and J. M. Tour, Nano Lett., 2013, 13, 72-78.

106 Y. Q. Jiang, Q. Zhou and L. Lin, IEEE 22nd International Conference on Micro Electro Mechanical Systems (MEMS), 2009.

107 B. Hsia, J. Marschewski, S. Wang, J. B. In, C. Carraro, D. Poulikakos, C. P. Grigoropoulos and R. Maboudian, Nanotechnology, 2014, 25, 055401.

108 A. M. Saleem, S. Shafiee, T. Krasia-Christoforou, I. Savva, G. Göransson, V. Desmaris and P. Enoksson, Sci. Technol. Adv. Mater., 2015, 16, 015007. 
109 F. Sammoura, T. Kwok Siong, A. Kozinda, Z. Xining and L. Liwei, IEEE 27th International Conference on Micro Electro Mechanical Systems (MEMS), 2014.

110 G. Eda and M. Chhowalla, Adv. Mater., 2010, 22, 2392-2415.

111 C. Liu, Z. Yu, D. Neff, A. Zhamu and B. Z. Jang, Nano Lett., 2010, 10, 4863-4868.

112 G. Eda, G. Fanchini and M. Chhowalla, Nat. Nanotechnol., 2008, 3, 270-274.

113 M. F. El-Kady, V. Strong, S. Dubin and R. B. Kaner, Science, 2012, 335, 1326-1330.

114 V. Strong, S. Dubin, M. F. El-Kady, A. Lech, Y. Wang, B. H. Weiller and R. B. Kaner, ACS Nano, 2012, 6, 13951403.

115 B. Zheng, T. Huang, L. Kou, X. Zhao, K. Gopalsamy and C. Gao, J. Mater. Chem. A, 2014, 2, 9736-9743.

116 P. Zhang, S. Sago, T. Yamaguchi and G. M. Anilkumar, J. Power Sources, 2013, 230, 225-229.

117 Z.-S. Wu, K. Parvez, A. Winter, H. Vieker, X. Liu, S. Han, A. Turchanin, X. Feng and K. Müllen, Adv. Mater., 2014, 26, 4552-4558.

118 B. $\mathrm{Hu}, \mathrm{K}$. Wang, L. Wu, S.-H. Yu, M. Antonietti and M.-M. Titirici, Adv. Mater., 2010, 22, 813-828.

119 J. Ding, H. Wang, Z. Li, A. Kohandehghan, K. Cui, Z. Xu, B. Zahiri, X. Tan, E. M. Lotfabad, B. C. Olsen and D. Mitlin, ACS Nano, 2013, 7, 11004-11015.

120 W. Chen and D. Deng, Carbon, 2015, 87, 70-77.

121 J. Jiang, J. Zhu, W. Ai, Z. Fan, X. Shen, C. Zou, J. Liu, H. Zhang and T. Yu, Energy Environ. Sci., 2014, 7, 26702679.

122 W.-J. Liu, K. Tian, Y.-R. He, H. Jiang and H.-Q. Yu, Environ. Sci. Technol., 2014, 48, 13951-13959.

123 C. Peng, X.-B. Yan, R.-T. Wang, J.-W. Lang, Y.-J. Ou and Q.-J. Xue, Electrochim. Acta, 2013, 87, 401-408.

124 V. Sahu, S. Grover, B. Tulachan, M. Sharma, G. Srivastava, M. Roy, M. Saxena, N. Sethy, K. Bhargava, D. Philip, H. Kim, G. Singh, S. K. Singh, M. Das and R. K. Sharma, Electrochim. Acta, 2015, 160, 244-253.

125 H. Wang, Z. Xu, A. Kohandehghan, Z. Li, K. Cui, X. Tan, T. J. Stephenson, C. K. King'ondu, C. M. B. Holt, B. C. Olsen, J. K. Tak, D. Harfield, A. O. Anyia and D. Mitlin, ACS Nano, 2013, 6, 5131-5141.

126 W. Deng, X. Ji, Q. Chen and C. E. Banks, RSC Adv., 2011, 1, 1171-1178.

127 M.-S. Balogun, W. Qiu, W. Wang, P. Fang, X. Lu and Y. Tong, J. Mater. Chem. A, 2015, 3, 1364-1387.

128 R. Levi, M. Bar-Sadan, A. Albu-Yaron, R. Popovitz-Biro, L. Houben, C. Shahar, A. Enyashin, G. Seifert, Y. Prior and R. Tenne, J. Am. Chem. Soc., 2010, 132, 11214-11222.

129 J. Bae, Y. Park, J. Yang, H. Kim and D. Kim, J. Solid State Electrochem., 2015, 19, 211-219.

130 C. Liu, Z. Li and Z. Zhang, Electrochim. Acta, 2014, 134, 8491.

131 E. T. T. Jones, O. M. Chyan and M. S. Wrighton, J. Am. Chem. Soc., 1987, 109, 5526-5528.

132 X. Lu, M. Yu, T. Zhai, G. Wang, S. Xie, T. Liu, C. Liang, Y. Tong and Y. Li, Nano Lett., 2013, 13, 2628-2633.
133 A. Achour, J. B. Ducros, R. L. Porto, M. Boujtita, E. Gautron, L. le Brizoual, M. A. Djouadi and T. Brousse, Nano Energy, 2014, 7, 104-113.

134 C. Zhu, P. Yang, D. Chao, X. Wang, X. Zhang, S. Chen, B. K. Tay, H. Huang, H. Zhang, W. Mai and H. J. Fan, Adv. Mater., 2015, 27, 4566-4571.

135 H. Cui, G. Zhu, X. Liu, F. Liu, Y. Xie, C. Yang, T. Lin, H. Gu and F. Huang, Adv. Sci., 2015, 1500126.

136 Q. Sun and Z.-W. Fu, Electrochem. Solid-State Lett., 2007, 10, A189-A193.

137 F. Gillot, J. Oro-Sole and M. R. Palacin, J. Mater. Chem., 2011, 21, 9997-10002.

138 D. K. Nandi, U. K. Sen, D. Choudhury, S. Mitra and S. K. Sarkar, ACS Appl. Mater. Interfaces, 2014, 6, 6606-6615.

139 X. Wang and Y. Li, J. Am. Chem. Soc., 2002, 124, 2880-2881. 140 Y. Gong, H. Wang, Z. Wei, L. Xie and Y. Wang, ACS Sustainable Chem. Eng., 2014, 2, 2435-2441.

141 C.-M. Wen, Z.-Y. Wen, Z. You, X.-F. Wang and G.-Z. Wang, Proc. Inst. Mech. Eng., Part N, 2014, 228, 77-81.

142 S. Jiang, T. Shi, D. Liu, H. Long, S. Xi, F. Wu, X. Li, Q. Xia and Z. Tang, J. Power Sources, 2014, 262, 494-500.

143 T. M. Dinh, K. Armstrong, D. Guay and D. Pech, J. Mater. Chem. A, 2014, 2, 7170-7174.

144 R. Warren, F. Sammoura, A. Kozinda and L. Liwei, IEEE 27th International Conference on Micro Electro Mechanical Systems (MEMS), 2014.

145 X. Wang, Y. Yin, X. Li and Z. You, J. Power Sources, 2014, 252, 64-72.

146 D. Aradilla, P. Gentile, G. Bidan, V. Ruiz, P. Gómez-Romero, T. J. S. Schubert, H. Sahin, E. Frackowiak and S. Sadki, Nano Energy, 2014, 9, 273-281.

147 D. Aradilla, P. Gentile, V. Ruiz, P. Gómez-Romero, J. Wimberg, B. Iliev, T. J. S. Schubert, S. Sadki and G. Bidan, Adv. Nat. Sci.: Nanosci. Nanotechnol., 2015, 6, 015004.

148 J. P. Alper, S. Wang, F. Rossi, G. Salviati, N. Yiu, C. Carraro and R. Maboudian, Nano Lett., 2014, 14, 1843-1847.

149 S. Chatterjee, R. Carter, L. Oakes, W. R. Erwin, R. Bardhan and C. L. Pint, J. Phys. Chem. C, 2014, 118, 10893-10902.

150 X. Lu, M. Yu, G. Wang, T. Zhai, S. Xie, Y. Ling, Y. Tong and Y. Li, Adv. Mater., 2013, 25, 267-272.

151 X. Lu, Y. Zeng, M. Yu, T. Zhai, C. Liang, S. Xie, M.-S. Balogun and Y. Tong, Adv. Mater., 2014, 26, 31483155.

152 K. Wang, H. Wu, Y. Meng and Z. Wei, Small, 2014, 10, 1431.

153 Z. Antar, J.-F. Feller and G. Vignaud, Polym. Adv. Technol., 2013, 24, 638-645.

154 D. Aradilla, G. Bidan, P. Gentile, P. Weathers, F. Thissandier, V. Ruiz, P. Gomez-Romero, T. J. S. Schubert, H. Sahin and S. Sadki, RSC Adv., 2014, 4, 26462-26467.

155 G. A. Snook and G. Z. Chen, J. Electroanal. Chem., 2008, 612, 140-146.

156 R. Ramya, R. Sivasubramanian and M. V. Sangaranarayanan, Electrochim. Acta, 2013, 101, 109-129. 
157 Y. Shi, L. Pan, B. Liu, Y. Wang, Y. Cui, Z. Bao and G. Yu, J. Mater. Chem. A, 2014, 2, 6086-6091.

158 J. Chen, H. Bi, S. Sun, Y. Tang, W. Zhao, T. Lin, D. Wan, F. Huang, X. Zhou, X. Xie and M. Jiang, ACS Appl. Mater. Interfaces, 2013, 5, 1408-1413.

159 X. Zang, Q. Zhou, J. Chang, Y. Liu and L. Lin, Microelectron. Eng., 2015, 132, 192-206.

160 H. Nan, W. Ma, Z. Gu, B. Geng and X. Zhang, RSC Adv., 2015, 5, 24607-24614.

161 X. Wang, Y. Yin, C. Hao and Z. You, Carbon, 2015, 82, 436445.

162 B. L. Corso, I. Perez, T. Sheps, P. C. Sims, O. T. Gül and P. G. Collins, Nano Lett., 2014, 14, 1329-1336.

163 K. Naoi, W. Naoi, S. Aoyagi, J.-I. Miyamoto and T. Kamino, Acc. Chem. Res., 2013, 46, 1075-1083.

164 J. Chen, C. Jia and Z. Wan, Electrochim. Acta, 2014, 121, 4956.

165 Z. Zeng, X. Long, H. Zhou, E. Guo, X. Wang and Z. Hu, Electrochim. Acta, 2015, 163, 107-115.

166 J. Duay, E. Gillette, R. Liu and S. B. Lee, Phys. Chem. Chem. Phys., 2012, 14, 3329-3337.

167 Z. Yu, C. Li, D. Abbitt and J. Thomas, J. Mater. Chem. A, 2014, 2, 10923-10929.

168 T. M. Dinh, A. Achour, S. Vizireanu, G. Dinescu, L. Nistor, K. Armstrong, D. Guay and D. Pech, Nano Energy, 2014, 10, 288-294.

169 D. Kim, J. Yun, G. Lee and J. S. Ha, Nanoscale, 2014, 6, 12034-12041.

170 D. Pech, M. Brunet, H. Durou, P. Huang, V. Mochalin, Y. Gogotsi, P.-L. Taberna and P. Simon, Nat. Nanotechnol., 2010, 5, 651-654.

171 W.-W. Liu, Y.-Q. Feng, X.-B. Yan, J.-T. Chen and Q.-J. Xue, Adv. Funct. Mater., 2013, 23, 4111-4122.

172 H. Zhang, G. Cao, Z. Wang, Y. Yang, Z. Shi and Z. Gu, Electrochem. Commun., 2008, 10, 1056-1059.

173 P. Yu, Y. Li, X. Zhao, L. Wu and Q. Zhang, Langmuir, 2014, 30, 5306-5313.

174 C. J. Raj, B. C. Kim, W.-J. Cho, W.-G. Lee, S.-D. Jung, Y. H. Kim, S. Y. Park and K. H. Yu, ACS Appl. Mater. Interfaces, 2015, 7, 13405-13414.

175 D. Aradilla, D. Gaboriau, G. Bidan, P. Gentile, M. Boniface, D. Dubal, P. Gomez-Romero, J. Wimberg, T. J. S. Schubert and S. Sadki, J. Mater. Chem. A, 2015, 3, 13978-13985.

176 Z. Wang, D. O. Carlsson, P. Tammela, K. Hua, P. Zhang, L. Nyholm and M. Strømme, ACS Nano, 2015, 9, 7563-7571.
177 W. G. Pell and B. E. Conway, J. Power Sources, 2004, 136, 334-345.

178 K. K. Lee, S. Deng, H. M. Fan, S. Mhaisalkar, H. R. Tan, E. S. Tok, K. P. Loh, W. S. Chin and C. H. Sow, Nanoscale, 2012, 4, 2958-2961.

179 Y. Zeng, Y. Han, Y. Zhao, Y. Zeng, M. Yu, Y. Liu, H. Tang, Y. Tong and X. Lu, Adv. Energy Mater., 2015, 5, 1402176.

180 Y. G. Zhu, Y. Wang, Y. Shi, J. Wong and H. Y. Yang, Nano Energy, 2014, 3, 46-54.

181 A. C. Pierre and G. M. Pajonk, Chem. Rev., 2002, 102, 42434266.

182 K. Qin, J. Kang, J. Li, C. Shi, Y. Li, Z. Qiao and N. Zhao, ACS Nano, 2015, 9, 481-487.

183 M. S. Kim, B. Hsia, C. Carraro and R. Maboudian, Carbon, 2014, 74, 163-169.

184 W. Liu, C. Lu, X. Wang, R. Y. Tay and B. K. Tay, ACS Nano, 2015, 9, 1528-1542.

185 J. Kim, W.-H. Khoh, B.-H. Wee and J.-D. Hong, RSC Adv., 2015, 5, 9904-9911.

186 Z. Peng, J. Lin, R. Ye, E. L. G. Samuel and J. M. Tour, ACS Appl. Mater. Interfaces, 2015, 7, 3414-3419.

187 J. Xu and G. Shen, Nano Energy, 2015, 13, 131-139.

188 S.-K. Kim, H.-J. Koo, A. Lee and P. V. Braun, Adv. Mater., 2014, 26, 5108-5112.

189 D. Kim, G. Lee, D. Kim and J. S. Ha, ACS Appl. Mater. Interfaces, 2015, 7, 4608-4615.

190 Y. Lim, J. Yoon, J. Yun, D. Kim, S. Y. Hong, S.-J. Lee, G. Zi and J. S. Ha, ACS Nano, 2014, 8, 11639-11650.

191 X. Dong, Z. Guo, Y. Song, M. Hou, J. Wang, Y. Wang and Y. Xia, Adv. Funct. Mater., 2014, 24, 3405-3412.

192 Y. Gogotsi, Nature, 2014, 509, 568-570.

193 A. B. Dalton, S. Collins, E. Munoz, J. M. Razal, V. H. Ebron, J. P. Ferraris, J. N. Coleman, B. G. Kim and R. H. Baughman, Nature, 2003, 423, 703.

194 D. Yu, K. Goh, H. Wang, L. Wei, W. Jiang, Q. Zhang, L. Dai and Y. Chen, Nat. Nanotechnol., 2014, 9, 555-562.

195 J. Ren, W. Bai, G. Guan, Y. Zhang and H. Peng, Adv. Mater., 2013, 25, 5965-5970.

196 Y. Ma, P. Li, J. W. Sedloff, X. Zhang, H. Zhang and J. Liu, ACS Nano, 2015, 9, 1352-1359.

197 S. Dai, H. Guo, M. Wang, J. Liu, G. Wang, C. Hu and Y. Xi, J. Mater. Chem. A, 2014, 2, 19665-19669.

198 B. Liu, B. Liu, X. Wang, D. Chen, Z. Fan and G. Shen, Nano Energy, 2014, 10, 99-107.

199 P. Xu, T. Gu, Z. Cao, B. Wei, J. Yu, F. Li, J.-H. Byun, W. Lu, Q. Li and T.-W. Chou, Adv. Energy Mater., 2014, 4, 1300759. 